\title{
Measurement of the $\bar{t} Z$ and $\bar{t} W$ cross sections in proton-proton collisions at $\sqrt{s}=13 \mathrm{TeV}$ with the ATLAS detector
}

\author{
M. Aaboud et al. \\ (ATLAS Collaboration)
}

(Received 14 January 2019; published 29 April 2019)

\begin{abstract}
A measurement of the associated production of a top-quark pair $(t \bar{t})$ with a vector boson $(W, Z)$ in proton-proton collisions at a center-of-mass energy of $13 \mathrm{TeV}$ is presented, using $36.1 \mathrm{fb}^{-1}$ of integrated luminosity collected by the ATLAS detector at the Large Hadron Collider. Events are selected in channels with two same- or opposite-sign leptons (electrons or muons), three leptons or four leptons, and each channel is further divided into multiple regions to maximize the sensitivity of the measurement. The $t \bar{t} Z$ and $t \bar{t} W$ production cross sections are simultaneously measured using a combined fit to all regions. The best-fit values of the production cross sections are $\sigma_{t \bar{t} Z}=0.95 \pm$ $0.08_{\text {stat }} \pm 0.10_{\text {syst }} \mathrm{pb}$ and $\sigma_{\bar{t} \bar{W}}=0.87 \pm 0.13_{\text {stat }} \pm 0.14_{\text {syst }} \mathrm{pb}$ in agreement with the Standard Model predictions. The measurement of the $t \bar{t} Z$ cross section is used to set constraints on effective field theory operators which modify the $t \bar{t} Z$ vertex.
\end{abstract}

DOI: 10.1103/PhysRevD.99.072009

\section{INTRODUCTION}

Properties of the top quark have been explored by the Large Hadron Collider (LHC) and previous collider experiments in great detail. The production cross sections of topquark pairs and single top quarks, as well as the top-quark mass, spin correlations and $W$ boson helicity fractions have all been measured. Other properties of the top quark are now becoming accessible, owing to the large centerof-mass energy and luminosity at the LHC. These include its coupling to the Higgs boson and electroweak neutralcurrent couplings, accessed by measurements of pairproduced top quarks in association with a Higgs boson [1-4] or a photon [5-8].

Measurements of top-quark pairs in association with a $Z$ or $W$ boson $(t \bar{t} Z$ and $t \bar{t} W$ ) provide a direct probe of the weak couplings of the top quark [9-11]. These couplings may be modified in the presence of physics beyond the Standard Model (BSM). Any deviations from the SM predictions due to BSM effects can be parametrized in a model-independent way using the framework of the Standard Model effective field theory (SMEFT) [12-14]. If no deviations are observed, measurements of the $t \bar{t} Z$ and $\bar{t} \bar{t} W$ production cross sections, $\sigma_{\bar{t} \bar{Z} Z}$ and $\sigma_{t \bar{t} W}$, can be used to set constraints on the weak couplings of the top quark in the

${ }^{*}$ Full author list given at the end of the article.

Published by the American Physical Society under the terms of the Creative Commons Attribution 4.0 International license. Further distribution of this work must maintain attribution to the author(s) and the published article's title, journal citation, and DOI. Funded by SCOAP ${ }^{3}$.
SMEFT context. The $t \bar{t} Z$ and $t \bar{t} W$ processes were observed by ATLAS $[15,16]$ and CMS $[17,18]$, with measured cross sections compatible with the SM prediction. At $13 \mathrm{TeV}$, ATLAS analyzed $3.2 \mathrm{fb}^{-1}$ of data using the same-sign dimuon, trilepton and tetralepton channels, and measured $\sigma_{\bar{t} \bar{Z} Z}=0.9 \pm 0.3 \mathrm{pb}$ and $\sigma_{t \bar{t} W}=1.5 \pm 0.8 \mathrm{pb}$, while CMS analyzed $35.9 \mathrm{fb}^{-1}$ and measured $0.99_{-0.13}^{+0.15} \mathrm{pb}$ and $0.77_{-0.16}^{+0.18} \mathrm{pb}$, respectively.

The production of $t \bar{t} Z$ and $t \bar{t} W$ is often an important background in searches involving final states with multiple leptons and $b$-quarks. These processes also constitute an important background in measurements of the associated production of the Higgs boson with top quarks.

This paper presents measurements of the $t \bar{t} Z$ and $t \bar{t} W$ cross sections using proton-proton $(p p)$ collision data at a center-of mass energy $\sqrt{s}=13 \mathrm{TeV}$ corresponding to an integrated luminosity of $36.1 \mathrm{fb}^{-1}$, collected by the ATLAS detector in 2015 and 2016. The final states of top-quark pairs produced in association with a $Z$ or a $W$ boson contain up to four isolated, prompt leptons. ${ }^{1}$ In this analysis, events with two opposite-sign (OS) or same-sign (SS) leptons, three leptons or four leptons are considered. The dominant backgrounds in these four channels are $Z+$ jets and $t \bar{t}$, events with nonprompt or misidentified leptons, $W Z$, and $Z Z$ production, respectively. An interpretation of the $t \bar{t} Z$ cross-section measurement in the SMEFT framework is also performed.

\footnotetext{
${ }^{1}$ In this paper, lepton is used to denote electron or muon, and prompt lepton is used to denote a lepton produced in a $Z$ or $W$ boson decay, or in the decay of a $\tau$-lepton which arises from a $Z$ or $W$ boson decay.
} 


\section{THE ATLAS DETECTOR}

The ATLAS detector [19] consists of three main subsystems: an inner tracking system, electromagnetic (EM) and hadronic calorimeters, and a muon spectrometer (MS). The inner detector (ID) consists of a high-granularity silicon pixel detector, including the insertable B-layer [20,21], which is the innermost layer of the tracking system, and a silicon microstrip tracker, together providing precision tracking in the pseudorapidity ${ }^{2}$ range $|\eta|<2.5$, followed by a transition radiation tracker covering $|\eta|<2$.0. All these systems are immersed in a $2 \mathrm{~T}$ magnetic field provided by a thin superconducting solenoid. The EM sampling calorimeter uses lead and liquid argon (LAr) and is divided into barrel $(|\eta|<1.475)$ and end cap $(1.375<$ $|\eta|<3.2)$ regions. Hadronic calorimetry is provided by a steel/scintillator-tile calorimeter, segmented into three barrel structures, in the range $|\eta|<1.7$, and by two copper/ LAr hadronic end cap calorimeters that cover the region $1.5<|\eta|<3.2$. The solid angle coverage is completed with forward copper/LAr and tungsten/LAr calorimeter modules, optimized for EM and hadronic measurements, respectively, covering the region $3.1<|\eta|<4$.9. The muon spectrometer measures the deflection of muons in the range $|\eta|<2.7$ using multiple layers of high-precision tracking chambers located in toroidal magnetic fields. The field integral of the toroids ranges between 2.0 and $6.0 \mathrm{Tm}$ for most of the detector. The muon spectrometer is also instrumented with separate trigger chambers covering $|\eta|<2$.4. A two-level trigger system [22], using custom hardware followed by a software-based trigger level, is used to reduce the event rate to an average of around $1 \mathrm{kHz}$ for offline storage.

\section{DATA AND SIMULATED EVENT SAMPLES}

The data were collected with the ATLAS detector during 2015 and 2016 at a proton-proton $(p p)$ collision energy of $13 \mathrm{TeV}$. The bunch spacing was $25 \mathrm{~ns}$ and the mean number of interactions per bunch crossing was 14 (25) in 2015 (2016). With strict data-quality requirements, the integrated luminosity considered corresponds to $36.1 \mathrm{fb}^{-1}$ [23,24].

Monte Carlo (MC) simulation samples are used to model the expected signal and background distributions in the different control, validation and signal regions described below. The heavy-flavor hadron decays involving $b$ - and $c$-quarks, particularly important in this measurement, were modeled using EvTGEN [25] v1.2.0, except for those processes modeled using the SHERPA [26] generator, as

\footnotetext{
${ }^{2}$ ATLAS uses a right-handed coordinate system with its origin at the nominal interaction point (IP) in the center of the detector and the $z$ axis along the beam pipe. The $x$ axis points from the IP to the center of the LHC ring, and the $y$ axis points upward. Cylindrical coordinates $(r, \phi)$ are used in the transverse plane, $\phi$ being the azimuthal angle around the $z$ axis. The pseudorapidity is defined in terms of the polar angle $\theta$ as $\eta=-\ln \tan (\theta / 2)$.
}

described below. In all samples the top-quark mass was set to $172.5 \mathrm{GeV}$, and the Higgs boson mass was set to $125 \mathrm{GeV}$. The response of the detector to stable ${ }^{3}$ particles was emulated by a dedicated simulation [27] based either fully on GEANT [28], or on a faster simulation [29] using a parametrized calorimeter response and GEANT for other detector systems. To account for additional $p p$ interactions from the same and nearby bunch crossings (pileup), minimum-bias interactions generated using PYTHIA v8.186 [30], referred to as PYTHIA 8 in the following, with the $\mathrm{A} 2$ [31] set of tuned MC parameters (A2 tune) were superimposed on the hard-scattering events.

Simulated events were corrected using per-event weights to describe the distribution of the average number of interactions per proton bunch crossing as observed in data. All samples were processed through the same reconstruction software as used for the data. Simulated events were corrected so that the object identification, reconstruction and trigger efficiencies, energy scales and energy resolutions match those determined from data control samples.

The associated production of a top-quark pair with one or two vector bosons was generated at next-to-leading order (NLO) with MADGRAPH5_aMC@NLO [32] (referred to in the following as MG5_aMC) version 2.3.2 interfaced to PYTHIA 8. The cross sections for the $t \bar{t} Z$ and $t \bar{t} W$ processes at $13 \mathrm{TeV}$, computed including NLO QCD and electroweak corrections using MG5_aMC, are $\sigma_{\bar{t} \bar{Z} Z}=0.88 \mathrm{pb}$ and $\sigma_{\bar{t} \bar{t} W}=0.60 \mathrm{pb}$ with an uncertainty of $\sim 12 \%$ [32-34]. The uncertainty is primarily due to higher-order QCD corrections, estimated by varying the renormalization $\left(\mu_{\mathrm{R}}\right)$ and factorization $\left(\mu_{\mathrm{F}}\right)$ scales. The $\gamma^{*}$ contribution and the $Z / \gamma^{*}$ interference were included in the $t \bar{t} Z$ samples, with the dilepton invariant mass $\left(m_{\ell \ell}\right)$ required to be above $5 \mathrm{GeV}$. The NNPDF2 . 3NLO parton distribution function (PDF) set [35] was used in the matrix-element (ME) computation. The A14 [36] set of tuned MC parameters (A14 tune) was used together with the NNPDF2 . 3LO PDF set [37] in the parton shower.

The $t$-channel production of a single top quark in association with a $Z$ boson $(t Z)$ was generated at leading order (LO) using MG5_aMC v2.2.3 interfaced to PYTHIA v6.427 [38], referred to as PYтHIA 6 in the following, with the CTEQ6L1 [39] PDF set and the Perugia2012 [40] set of tuned MC parameters at NLO in QCD. The fourflavor scheme was used in the generation, and the sample was normalized using the cross section computed at NLO in QCD using MG5_aMC.

The production of a single top quark together with a $W$ and a $Z$ boson $(t W Z)$ was generated with MG5_aMC v2.3.3 using the NNPDF3. ONLO PDF set [35]. The generation was performed at NLO in QCD using the five-flavor scheme. Diagrams containing a top-quark pair were

\footnotetext{
${ }^{3} \mathrm{~A}$ particle is considered stable if $c \tau \geq 1 \mathrm{~cm}$.
} 
removed to avoid overlap with the $t \bar{t} Z$ process. The parton shower was modeled by PYTHIA 8 with the A14 tune. The sample was normalized using the NLO cross section obtained from the generator.

Events containing $Z$ or $W$ bosons with associated jets were simulated using the SHERPA 2.2.1 event generator. The matrix-element calculation was performed using CoMIX [41] and OpENLoOps [42] for up to two partons at NLO and four partons at LO, and merged with the SHERPA parton shower [43] according to the ME+PS@NLO prescription [44]. The NNPDF3. ONNLO PDF set was used in conjunction with dedicated parton-shower tuning developed by the SHERPA authors. The $Z / W+$ jets samples were normalized to next-to-next-to-leading-order (NNLO) QCD cross sections for $Z / W$ production calculated by the FEWZ program [45].

Diboson processes with four charged leptons $(4 \ell)$, three charged leptons and one neutrino $(\ell \ell \ell \nu)$ or two charged leptons and two neutrinos $(\ell \ell \nu \nu)$ were simulated using the SHERPA 2.1.1 generator. The matrix elements included all diagrams with four electroweak vertices. They were calculated including up to three partons at LO, and the CT1O PDF set [46] was used in conjunction with a dedicated parton-shower tune developed by the SHERPA authors. The invariant mass of any two opposite-sign, same-flavor (OSSF) leptons was required to be greater than $5 \mathrm{GeV}$ in the generated events.

The production of three massive vector bosons with subsequent leptonic decays of all three bosons was modeled at LO with the SHERPA 2.1.1 generator and the CT10 PDF set. Up to two additional partons were included in the matrix element at LO and the full NLO accuracy was used for the inclusive process.

Electroweak processes involving the vector-boson scattering (VBS) diagram and producing two same-sign leptons, two neutrinos and two partons were modeled using SHERPA 2.1.1 at LO accuracy and the CT10 PDF set. Processes of orders four and six in the electroweak coupling constant were considered, and up to one additional parton was included in the matrix element. Other VBS processes are found to be negligible in the analysis regions considered.

The PowHEg-Box [47-50] v2 generator with the NNPDF3. ONLO PDF set was used for the generation of $t \bar{t}$ events. The parton shower and the underlying event were simulated using PYTHIA 8 with the NNPDF2 . 3LO PDF set and the corresponding A14 tune. The $h_{\text {damp }}$ parameter, which controls the transverse momentum of the first gluon emission beyond the Born configuration, was set to 1.5 times the top-quark mass. The $t \bar{t}$ samples were normalized to the NNLO cross-section predictions, including softgluon resummation to next-to-next-to-leading-logarithm order, as calculated with the Top++2.0 [51] program.

Electroweak $s$-channel and $t$-channel single-topquark events, and $W t$ final states, were generated with
Powheg-Box v1, and the parton shower modeled by PYTHIA v6.428. The CT10 PDF set was used for $s$-channel production and $W t$ events, while for $t$-channel production the four-flavor scheme was used for the NLO matrix element calculations together with the fixed four-flavor PDF set CT10f4. Diagram removal was employed to remove the overlap between $t \bar{t}$ and $W t$ production [52]. The single-top-quark samples were normalized to the cross sections computed at NLO reported in Refs. $[53,54]$ for the $s$ - and $t$-channels and at NLO with next-to-next-to-leadinglogarithm soft-gluon corrections for $W t$ production [55].

Samples of $t \bar{t}$ events produced in association with a Higgs boson $(t \bar{t} H)$ were generated using NLO matrix elements in MG5_aMC with the NNPDF3. ONLO PDF set and interfaced to PYTHIA 8 for the modeling of the parton shower. Higgs boson production via gluon-gluon fusion ( $\mathrm{ggF}$ ) and vector-boson fusion (VBF) was generated using the PowHEG-Box v2 generator with the CT10 PDF set. The parton shower and underlying event were simulated using PYTHIA 8 with the CTEQ6L1 PDF set and AZNLO tune. Higgs boson production with a vector boson was generated at LO using PYTHIA 8 with the NNPDF2 . 3LO PDF. All Higgs boson samples were normalized using theoretical calculations presented in Ref. [34].

The SM production of three and four top quarks was generated at LO with MG5_aMC+PYTHIA 8, using the A14 tune together with the NNPDF2 . 3LO PDF set. The samples were normalized using cross sections computed at NLO [56,57].

The events with a $Z$ or $W$ boson in association with a photon were simulated with up to three partons at $\mathrm{LO}$ using SHERPA 2.1.1 (ME + PS mode) and the CT10 PDF set. They were normalized to the LO cross section obtained from the generator. The $t \bar{t} \gamma$ process was generated at $\mathrm{LO}$ with MG5_aMC+PythiA 8, using the A14 tune together with the NNPDF2.3LO PDF set and normalized to the NLO cross section. Events in the $t \bar{t}$ sample with radiated photons of high transverse momentum were vetoed to avoid overlap with those from the $t \bar{t} \gamma$ sample.

\section{OBJECT RECONSTRUCTION}

Electron candidates [58] are reconstructed from energy deposits (clusters) in the EM calorimeter that are associated with reconstructed tracks in the ID. Electrons are required to pass the "medium" likelihood identification requirements described in Ref. [58]. In the SS dilepton channel, the "tight" likelihood requirement is used instead. The electrons are also required to have transverse momentum $p_{\mathrm{T}}>7 \mathrm{GeV}$ and $\left|\eta_{\text {cluster }}\right|<2.47$, where $\eta_{\text {cluster }}$ is the pseudorapidity of the calorimeter energy deposit associated with the electron candidate. Candidates in the EM calorimeter barrel/end cap transition region $1.37<\left|\eta_{\text {cluster }}\right|<$ 1.52 are excluded. 
Muon candidates are reconstructed from a fit to track segments in the various layers of the muon spectrometer, matched with tracks identified in the inner detector. Muons are required to have $p_{\mathrm{T}}>7 \mathrm{GeV}$ and $|\eta|<2.5$ and to pass the medium identification requirements defined in Ref. [59]. The medium criteria include requirements on the numbers of hits in the ID and MS as well as a compatibility requirement between momentum measurements in the ID and MS. They provide a high efficiency and purity of selected muons. Electron candidates sharing a track with a muon candidate are removed.

To reduce the background due to nonprompt leptons from hadron decays, photon conversions or jets misidentified as leptons (labeled as "fake leptons" throughout this paper), electron and muon candidates are required to be isolated. In the OS dilepton and the tetralepton channels, as well as in those trilepton regions that target the $t \bar{t} Z$ process, the total sum of the track transverse momenta in a surrounding cone of size $\Delta R_{\eta} \equiv \sqrt{(\Delta \eta)^{2}+(\Delta \phi)^{2}}=$ $\min \left(10 \mathrm{GeV} / p_{\mathrm{T}}, r_{e, \mu}\right)$, excluding the track of the candidate, is required to be less than $6 \%$ of the candidate $p_{\mathrm{T}}$, where $r_{e}=0.2$ and $r_{\mu}=0.3$. In addition, the sum of the cluster transverse energies in the calorimeter within a cone of size $\Delta R_{\eta}=0.2$ around any electron candidate, excluding energy deposits of the candidate itself, is required to be less than $6 \%$ of the candidate $p_{\mathrm{T}}$.

In the SS dilepton channel and those trilepton regions targeting the $t \bar{t} W$ process, where the fake-lepton background is particularly important, tighter isolation requirements are imposed on candidate leptons. A multivariate discriminant is built to distinguish prompt leptons from leptons arising from heavy-hadron decays inside jets [1]. The discriminant uses information from charged-particle tracks in a cone around the lepton candidate. Jets are reconstructed from these tracks to obtain a track jet, and the discriminant is constructed from information such as the angular distance between the lepton and the track jet, the number of tracks in the track jet and the ratio of the lepton candidate $p_{\mathrm{T}}$ to the track-jet $p_{\mathrm{T}}$. The rejection factor obtained for leptons from $b$-hadron decays is about 20, while the prompt-lepton efficiency is about $85 \%$ (80\%) for $p_{\mathrm{T}} \sim 20 \mathrm{GeV}$ and reaches a plateau of $\sim 98 \%$ (96\%) for muons (electrons) at high $p_{\mathrm{T}}$. Simulated events are corrected to account for differences in the prompt-lepton tagging discriminant between data and simulation.

Another important background in the SS dilepton channel arises from electrons with misidentified charge. To suppress this background, another multivariate discriminant is used, which takes as inputs various track and cluster properties of the electron candidates [1]. The discriminant provides a $95 \%$ efficiency for electrons with correct charge reconstruction while achieving a rejection factor of $\sim 17$ for electrons with misidentified charge that pass the tight likelihood identification requirement. Correction factors are applied to selected electrons to match the efficiency of the discriminant in simulation to that measured in data.

For both the electrons and muons, the longitudinal impact parameter of the associated track relative to the primary vertex ${ }^{4} z_{0}$, is required to satisfy $\left|z_{0} \sin \theta\right|<0.5 \mathrm{~mm}$. The significance of the transverse impact parameter $d_{0}$ is required to satisfy $\left|d_{0}\right| / \sigma\left(d_{0}\right)<5$ for electrons and $\left|d_{0}\right| / \sigma\left(d_{0}\right)<3$ for muons, where $\sigma\left(d_{0}\right)$ is the uncertainty in $d_{0}$.

Jets are reconstructed using the anti- $k_{t}$ algorithm $[60,61]$ with radius parameter $R=0.4$, starting from topological energy clusters in the calorimeters [62]. The effect of pileup on jet energies is accounted for by a jet-area-based correction [63] and the energy resolution of the jets is improved by using global sequential corrections [64]. Jets are calibrated to the hadronic energy scale using energyand pseudorapidity-dependent calibration factors derived from data. Jets are accepted if they fulfill the requirements $p_{\mathrm{T}}>25 \mathrm{GeV}$ and $|\eta|<2.5$. To reduce the contribution from jets associated with pileup, jets with $p_{\mathrm{T}}<60 \mathrm{GeV}$ and $|\eta|<2.4$ are required to satisfy pileup rejection criteria (JVT), based on a multivariate combination of track-based variables [65].

Jets are tagged as likely to contain $b$-hadrons ( $b$-tagged) with a multivariate discriminant making use of the long lifetime, large decay multiplicity, hard fragmentation and high mass of $b$-hadrons [66]. For the working point used in this analysis, the average efficiency for correctly tagging a $b$-jet is approximately $77 \%$, as determined in simulated $t \bar{t}$ events. In simulation, the tagging algorithm gives a rejection factor of 134 against light-quark and gluon jets, and 6.2 against charm-quark jets. The $b$-tagging efficiency and mistagging rates in simulation are corrected to reproduce those in data [66].

The missing transverse momentum $\mathbf{p}_{\mathrm{T}}^{\text {miss }}$, with magnitude $E_{\mathrm{T}}^{\text {miss }}$, is a measure of the transverse momentum imbalance due to particles escaping detection. It is computed [67] as the negative sum of the transverse momenta of all electrons, muons and jets and an additional soft term. The soft term is constructed from all tracks that are associated with the primary vertex but not with any lepton or jet. In this way, the $E_{\mathrm{T}}^{\text {miss }}$ is adjusted for the best calibration of the jets and the other identified objects, while maintaining pileup independence in the soft term $[67,68]$.

To prevent double-counting of electron energy deposits as jets, the closest jet within $\Delta R_{y}=0.2$ of a reconstructed electron is removed, where $\Delta R_{y} \equiv \sqrt{(\Delta y)^{2}+(\Delta \phi)^{2}}$ and $y$ is the rapidity of the electron. If the nearest jet surviving the above criterion is within $\Delta R_{y}=0.4$ of an electron, the

\footnotetext{
${ }^{4} \mathrm{~A}$ primary vertex candidate is defined as a vertex with at least two associated tracks, consistent with the beam collision region. The vertex candidate with the largest sum of squared transverse momenta of its associated tracks is taken as the primary vertex.
} 
electron is discarded to ensure that selected electrons are sufficiently separated from nearby jet activity. To reduce the background from muons originating from heavy-flavor particle decays inside jets, muons are removed if they are separated from the nearest jet by $\Delta R_{y}<0.4$. However, if this jet has fewer than three associated tracks, the muon is kept and the jet is removed instead; this ensures that highenergy muons undergoing significant energy loss in the calorimeter are retained.

\section{EVENT SELECTION AND BACKGROUND ESTIMATION}

Table I lists the analysis channels and the targeted decay modes of the $t \bar{t} Z$ and $t \bar{t} W$ processes. Each channel is divided into multiple analysis regions in order to enhance the sensitivity to the signal. Simultaneous fits to the signal regions and dedicated control regions are performed to extract the cross sections for $t \bar{t} Z$ and $t \bar{t} W$ production.

Only events collected using single-electron or singlemuon triggers are accepted. Events are required to have at least one reconstructed primary vertex. In all regions considered, at least one reconstructed lepton with $p_{\mathrm{T}}>$ $27 \mathrm{GeV}$ is required to match $\left(\Delta R_{\eta}<0.15\right)$ a lepton with the same flavor reconstructed by the trigger algorithm. Four channels are defined: same-sign dilepton, opposite-sign dilepton, trilepton and tetralepton.

The shapes of background distributions containing prompt leptons are modeled by simulation. The normalizations for the $W Z$ and $Z Z$ processes, as well as the production of a $Z$ boson in association with heavy-flavor (HF) jets, are taken from data control regions as defined in this section and which are included in the fit discussed in Sec. VII. The yields in these data control regions are extrapolated to the signal regions using simulation. Systematic uncertainties in the extrapolation are taken into account in the overall uncertainty in the background estimate.

The contribution from events containing an electron with misidentified charge (referred to as "charge-flip" in the following) is estimated from data. The charge-flip probability is extracted in events containing a pair of electrons with $m_{\ell \ell}$ close to the $Z$ boson mass. It is parametrized

TABLE I. List of $t \bar{t} W$ and $t \bar{t} Z$ decay modes and analysis channels targeting them. The symbols $b$ and $\nu$ denote a bottom quark or antiquark and neutrino or antineutrino, respectively, with charge conjugation implied.

\begin{tabular}{llcl}
\hline \hline Process & \multicolumn{1}{c}{$\bar{t}$ decay } & Boson decay & Channel \\
\hline $\bar{t} \bar{t} W$ & $\left(\ell^{ \pm} \nu b\right)(q \bar{q} b)$ & $\ell^{ \pm} \nu$ & SS dilepton \\
& $\left(\ell^{ \pm} \nu b\right)\left(\ell^{\mp} \nu b\right)$ & $\ell^{ \pm} \nu$ & Trilepton \\
\hline $\bar{t} \bar{t} Z$ & $(q \bar{q} b)(q \bar{q} b)$ & $\ell^{+} \ell^{-}$ & OS dilepton \\
& $\left(\ell^{ \pm} \nu b\right)(q \bar{q} b)$ & $\ell^{+} \ell^{-}$ & Trilepton \\
& $\left(\ell^{ \pm} \nu b\right)\left(\ell^{\mp} \nu b\right)$ & $\ell^{+} \ell^{-}$ & Tetralepton \\
\hline \hline
\end{tabular}

in $p_{\mathrm{T}}$ and $\eta$ and is found to range from around $0.01 \%$ to $2 \%$, increasing with $p_{\mathrm{T}}$ and $\eta$, for electrons passing the identification and isolation criteria applied in the SS dilepton channel. The probability is extracted by maximizing a likelihood which relates the number of events in which the two electrons have the same charge to the total number of observed events. The background contribution from events other than charge-flip electrons in the samesign region is estimated from a sideband of the $m_{\ell \ell}$ distribution and subtracted before performing the likelihood fit.

The charge-flip background contribution in any SS dilepton region is estimated by constructing a control region with identical requirements, but without any requirement on the lepton charge, and applying the appropriate charge-flip probabilities.

Background sources involving one or more fake leptons are modeled using data events from dedicated regions. For the SS dilepton ( $2 \ell-\mathrm{SS})$ and trilepton channels the fakelepton background is estimated using the matrix method [69]. The matrix method makes use of events with the same selection as the region of interest, but for which the electron identification criteria are relaxed to the "loose" likelihood requirement of Ref. [58], and neither electrons nor muons are required to be isolated. These leptons are referred to as loose leptons, whereas leptons satisfying the full set of identification and isolation criteria of Sec. IV are referred to as tight leptons. The fake-lepton background in any region of interest is obtained from the aforementioned events using efficiencies for prompt and fake leptons to satisfy the tight criteria.

The lepton efficiencies are extracted in control regions with a likelihood fit, by using the model of the matrix method, and assuming that the number of events with two fake leptons is negligible. The control regions are defined in dilepton events, separately for events with exactly one $b$-tagged jet and $\geq 2 b$-tagged jets. The prompt lepton efficiencies are measured in inclusive OSSF events, while fake-lepton efficiencies are measured in events with samesign leptons. Both the prompt and fake-lepton efficiencies are parametrized as a function of the lepton $p_{\mathrm{T}}$. The measurement of fake-lepton efficiencies is performed after subtracting the estimated contribution from charge-flip events. Contributions from processes with two prompt

TABLE II. Summary of the event selection requirements in the OS dilepton signal regions.

\begin{tabular}{lccc}
\hline \hline Variable & $2 \ell-\mathrm{Z}-6 \mathrm{j} 1 \mathrm{~b}$ & $2 \ell-\mathrm{Z}-5 \mathrm{j} 2 \mathrm{~b}$ & $2 \ell-\mathrm{Z}-6 \mathrm{j} 2 \mathrm{~b}$ \\
\hline Leptons & $=2$, same flavor and opposite sign \\
$m_{\ell \ell}$ & \multicolumn{4}{c}{$\left|m_{\ell \ell}-m_{Z}\right|<10 \mathrm{GeV}$} \\
$p_{\mathrm{T}}$ (leading lepton) & \multicolumn{4}{c}{$>30 \mathrm{GeV}$} \\
$p_{\mathrm{T}}$ (subleading lepton) & 1 & $>15 \mathrm{GeV}$ & \\
$n_{b \text {-tags }}$ & $\geq 6$ & 52 & $\geq 2$ \\
$n_{\text {jets }}$ & & & $\geq 6$ \\
\hline \hline
\end{tabular}




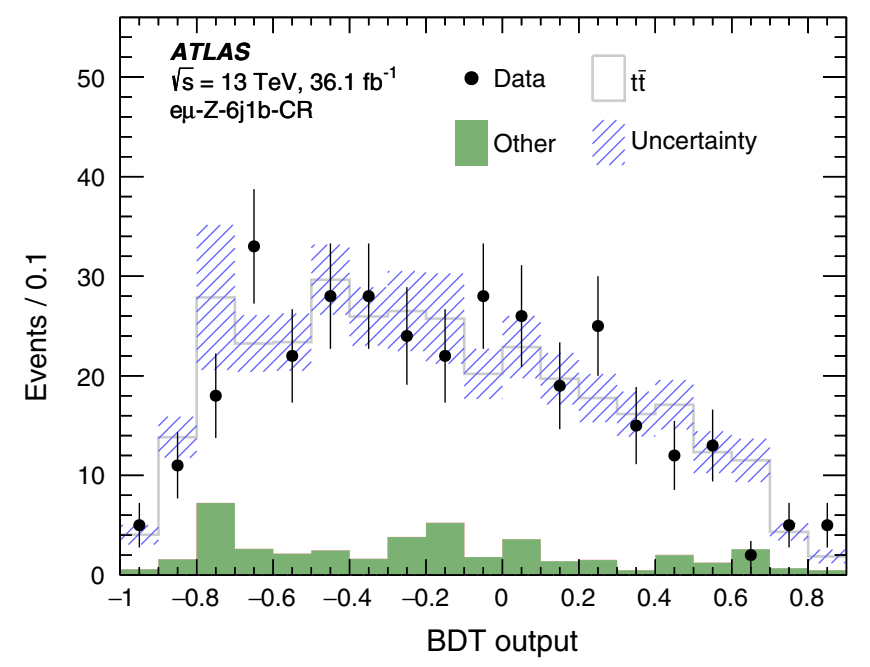

(a)

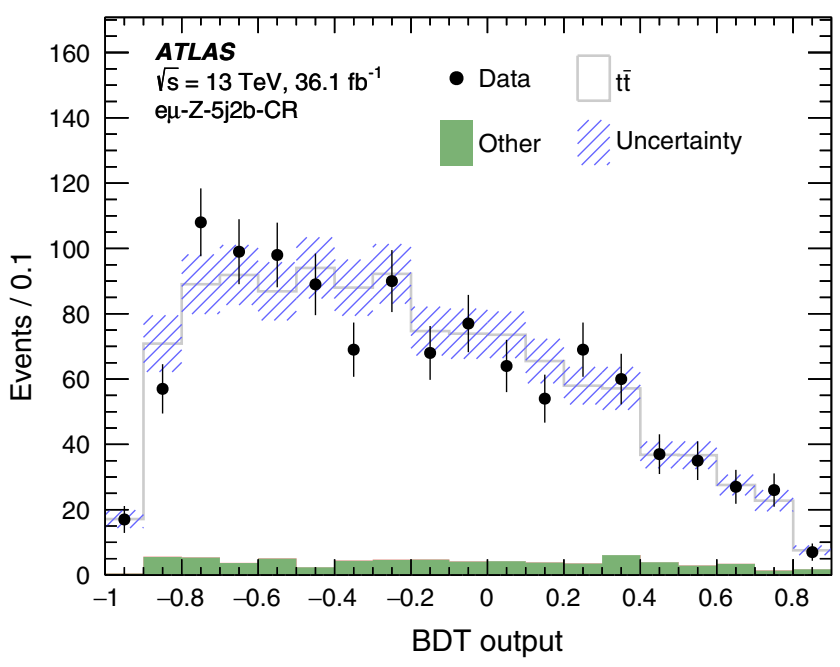

(b)

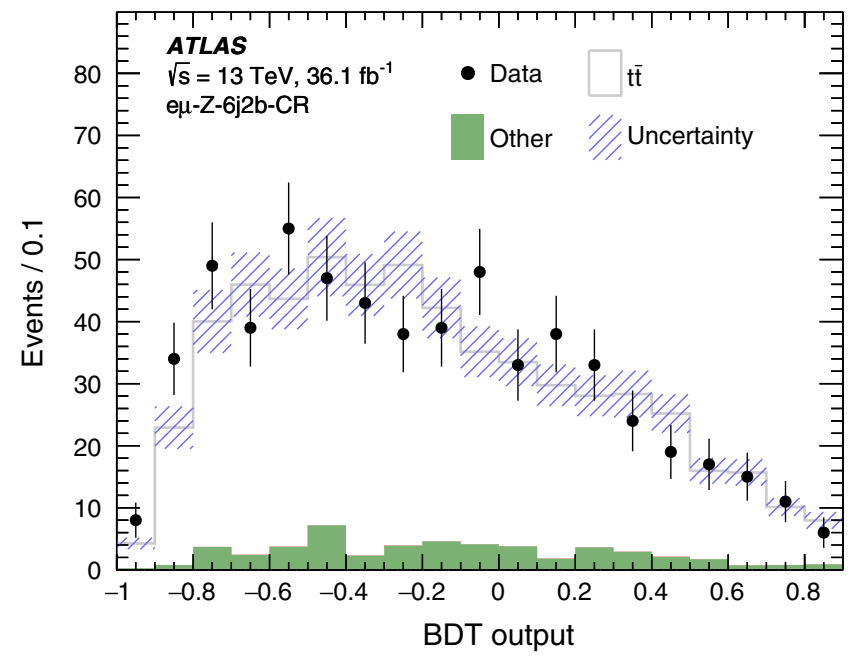

(c)

FIG. 1. The BDT distributions for the $t \bar{t}$ control regions (a) $2 \ell-Z-6 \mathrm{j} 1 \mathrm{~b}$, (b) $2 \ell-Z-5 \mathrm{j} 2 \mathrm{~b}$, (c) $2 \ell-Z-6 \mathrm{j} 2 \mathrm{~b}$. The shaded band represents the total uncertainty. The "Other" background contains SM processes with small cross sections producing two opposite-sign prompt leptons, including the $t \bar{t} Z$ process, whose contribution is negligible.

same-sign leptons or one real lepton and a photon conversion (arising mainly from $t \bar{t} \gamma$ production) are estimated from simulation and are also subtracted. The prompt and fake-lepton efficiencies are extracted separately for the regions targeting $t \bar{t} Z$ and those targeting $t \bar{t} W$, due to the different lepton isolation requirements applied in the two sets of regions.

In the tetralepton channel, the matrix method is not used due to the small number of events in data with four selected leptons. Instead, the contribution from

TABLE III. Summary of the event selection requirements in the SS dilepton signal regions.

\begin{tabular}{|c|c|c|c|c|}
\hline Requirement & $2 \ell-\mathrm{SS}(\mathrm{p}, \mathrm{m})-1 b$ & $2 e-\mathrm{SS}(\mathrm{p}, \mathrm{m})-2 b$ & $e \mu-\mathrm{SS}(\mathrm{p}, \mathrm{m})-2 b$ & $2 \mu-\mathrm{SS}(\mathrm{p}, \mathrm{m})-2 b$ \\
\hline$n_{b \text {-tags }}$ & $=1$ & $\geq 2$ & $\geq 2$ & $\geq 2$ \\
\hline$E_{\mathrm{T}}^{\mathrm{miss}}$ & $>40 \mathrm{GeV}$ & $>40 \mathrm{GeV}$ & $>40 \mathrm{GeV}$ & $>20 \mathrm{GeV}$ \\
\hline$H_{\mathrm{T}}$ & \multicolumn{4}{|c|}{$>240 \mathrm{GeV}$} \\
\hline$p_{\mathrm{T}}$ (leading lepton) & \multicolumn{4}{|c|}{$>27 \mathrm{GeV}$} \\
\hline$p_{\mathrm{T}}$ (subleading lepton) & \multicolumn{4}{|c|}{$>27 \mathrm{GeV}$} \\
\hline$n_{\text {jets }}$ & $\geq 4$ & $\geq 4$ & $\geq 4$ & $\geq 2$ \\
\hline$Z$ veto & \multicolumn{4}{|c|}{$\left|m_{\ell \ell}-m_{Z}\right|>10 \mathrm{GeV}$ in the $2 e$ and $2 \mu$ regions } \\
\hline
\end{tabular}


backgrounds containing fake leptons is estimated from simulation and corrected with scale factors determined in control regions. The contributions from events containing a photon conversion (denoted by $\gamma+X$ ) in the SS dilepton and trilepton channels are estimated from simulation and scaled with these correction factors, obtained separately for lepton type and origin.

\section{A. Opposite-sign dilepton analysis}

The OS dilepton analysis targets the $t \bar{t} Z$ process, where both top quarks decay hadronically and the $Z$ boson decays to a pair of leptons (electrons or muons). Events are required to have exactly two OSSF leptons. Events with additional isolated leptons are rejected. The invariant mass of the lepton pair is required to be in the $Z$ boson mass window, $\left|m_{\ell \ell}-m_{Z}\right|<10 \mathrm{GeV}$. The leading (subleading) lepton is required to have a transverse momentum of at least 30 (15) GeV.

The OS dilepton analysis is affected by large backgrounds from $Z+$ jets or $t \bar{t}$ production, both characterized by the presence of two leptons. In order to improve the signal-to-background ratio and constrain these backgrounds from data, three separate analysis regions are considered, depending on the number of jets $\left(n_{\text {jets }}\right)$ and number of $b$-tagged jets $\left(n_{b \text {-tags }}\right): 2 \ell-\mathrm{Z}-5 \mathrm{j} 2 \mathrm{~b}, 2 \ell-\mathrm{Z}-6 \mathrm{j} 1 \mathrm{~b}$ and $2 \ell-\mathrm{Z}-6 \mathrm{j} 2 \mathrm{~b}$. The signal region requirements are summarized in Table II. In signal region $2 \ell-Z-5 \mathrm{j} 2 \mathrm{~b}$, exactly five jets are required, of which at least two must be $b$-tagged. In $2 \ell$ $\mathrm{Z}-6 \mathrm{j} 1 \mathrm{~b}(2 \ell-\mathrm{Z}-6 \mathrm{j} 2 \mathrm{~b})$, at least six jets are required with exactly one (at least two) being $b$-tagged jets.

In order to separate signal from background, boosted decision trees (BDTs) are used. The BDTs are constructed and trained separately for each region against all the contributing backgrounds, using as input 15,14 and 17 variables for $2 \ell-\mathrm{Z}-6 \mathrm{j} 1 \mathrm{~b}, 2 \ell-\mathrm{Z}-5 \mathrm{j} 2 \mathrm{~b}$ and $2 \ell-\mathrm{Z}-6 \mathrm{j} 2 \mathrm{~b}$, respectively. Fourteen of the variables are common to the three regions. The details of the variables used are given in Table XI in the Appendix. In all three regions, the variables with the largest discriminative power are found to be

(i) the $\eta$ of the dilepton system,

(ii) the scalar sum of transverse momenta of all jets divided by the sum of their energies,

(iii) the first Fox-Wolfram moment $H_{1}$ [70].

Each of the signal regions is further divided into 19 equal-size bins of the BDT distribution. To avoid relying on simulation for the normalization of $Z+\mathrm{HF}$ jet production, the $Z+$ jets background is constrained by using events with low values of the BDT discriminant. The simulated $Z+$ jets background is split into three components, $Z+0 \mathrm{HF}$, $Z+1 \mathrm{HF}$ and $Z+2 \mathrm{HF}$, depending on the number of reconstructed jets which are matched to a generator-level $b$ - or $c$-hadron (heavy-flavor, or HF jets). The normalization factors of the $Z+1 \mathrm{HF}$ and $Z+2 \mathrm{HF}$ components of the $Z+$ jets background are determined from the fit to data, as described in Sec. VII, while the normalization of the $Z+0$ HF component is taken from simulation.

A data-driven method is used to estimate the $t \bar{t}$ background in the OS dilepton signal regions. Control regions are defined which are identical to the signal regions, except that the requirement of two leptons with the same flavor and opposite sign is replaced by the requirement of two leptons with different flavors and opposite sign. In this manner, three regions enriched in $t \bar{t}$ background are obtained. The number of $t \bar{t}$ events in each sameflavor dilepton region is estimated from corresponding

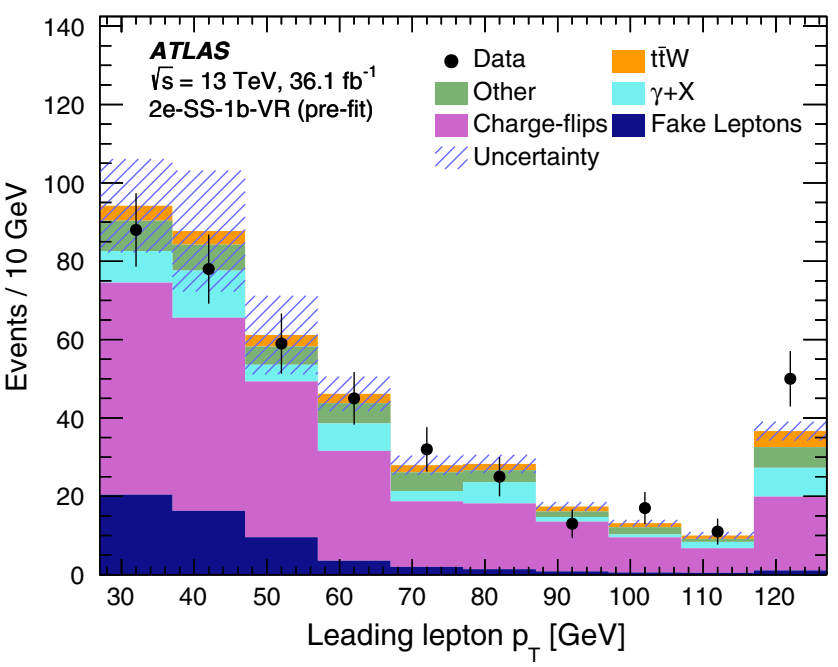

(b)

FIG. 2. Distributions in the $2 e-\mathrm{SS}-1 \mathrm{~b}-\mathrm{VR}$ validation region: (a) the invariant mass $m_{\ell \ell}$ of the lepton pair and (b) leading lepton transverse momentum $p_{\mathrm{T}}$. The shaded band represents the total uncertainty. The "Other" background contains SM processes with small cross sections producing two same-sign prompt leptons. The last bin in each of the distributions includes the overflow. 
opposite-flavor regions, corrected for non- $t \bar{t}$ backgrounds and differences in contributions from leptonic $\tau$-lepton decays. This procedure is applied to each bin of the distribution under consideration. Figure 1 shows the BDT distributions for the $t \bar{t}$ control regions. Agreement between the data and the expectation is observed.

\section{B. Same-sign dilepton analysis}

The SS dilepton signal regions target the $t \bar{t} W$ process. Events are required to have two lepton candidates with the same sign and $p_{\mathrm{T}}>27 \mathrm{GeV}$. The scalar sum of the $p_{\mathrm{T}}$ of selected leptons and jets, $H_{\mathrm{T}}$, is required to be above $240 \mathrm{GeV}$. Events containing additional loose leptons (with $p_{\mathrm{T}}>7 \mathrm{GeV}$ ) are vetoed. Twelve signal regions are defined

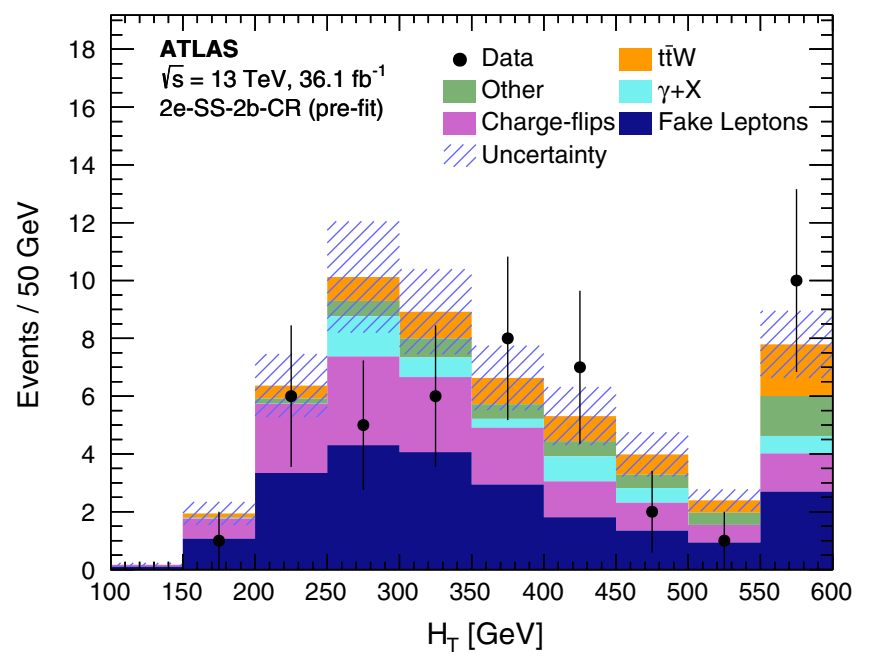

(a) in total, categorized by the number of $b$-tagged jets (one or $\geq 2$ ) as well as the charge and the flavor of the selected leptons. The signal regions are denoted by $2 \ell$-SSp- $1 b, 2 \ell-$ $\mathrm{SSm}-1 b, 2 \ell-\mathrm{SSp}-2 b$ and $2 \ell$-SSm- $2 b$, where "p" or "m" indicates the charge of the selected leptons. Considering separate signal regions for positive and negative charges of the leptons increases the sensitivity of the analysis since $t \bar{t} W$ events are preferentially produced with positively charged $W$ bosons, while the fake-lepton background and other processes such as $t \bar{t} Z$ and $t \bar{t} H$ are expected to be charge symmetric.

The event selection requirements in the SS dilepton regions are summarized in Table III. The presence of at least four jets and $E_{\mathrm{T}}^{\text {miss }}>40 \mathrm{GeV}$ is required in all signal regions except $2 \mu$-SSp- $2 b$ and $2 \mu$-SSm- $2 b$. In these

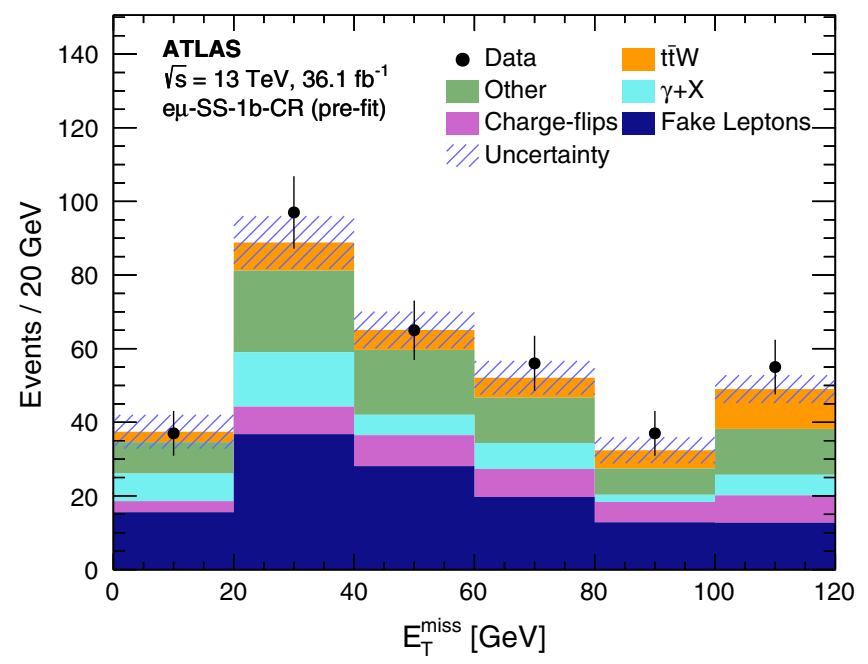

(b)

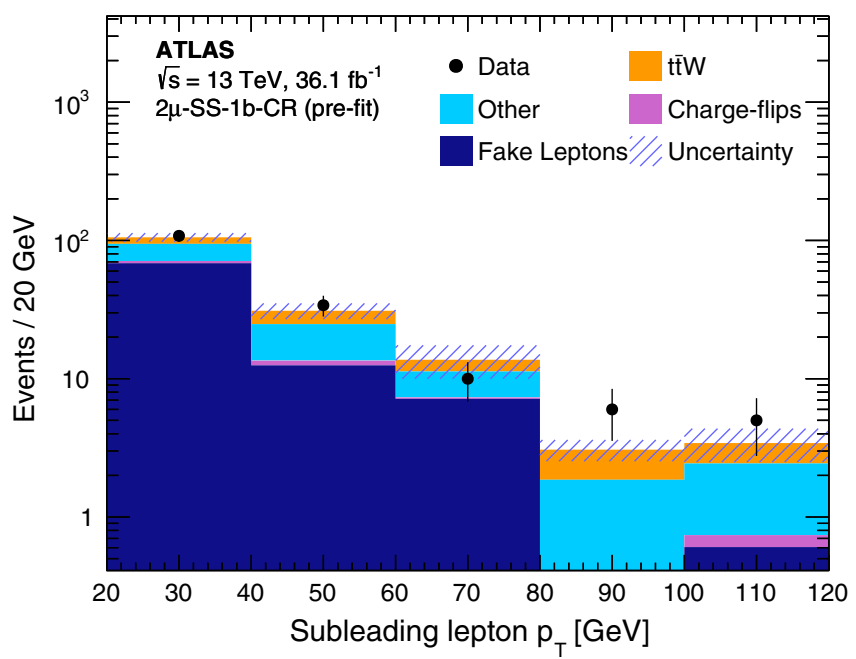

(c)

FIG. 3. Distributions in fake-lepton control regions: (a) scalar sum of transverse momenta of leptons and jets, $H_{\mathrm{T}}$, in the $2 e$-SS-2b-CR region, (b) missing transverse momentum, $E_{\mathrm{T}}^{\text {miss }}$, in the $e \mu$-SS- $1 b$-CR region and (c) subleading lepton transverse momentum, $p_{\mathrm{T}}$, in the $2 \mu$-SS- $1 b$-CR region. The shaded band represents the total uncertainty. The "Other" background contains SM processes with small cross sections producing two same-sign prompt leptons. The last bin in each of the distributions includes the overflow. 


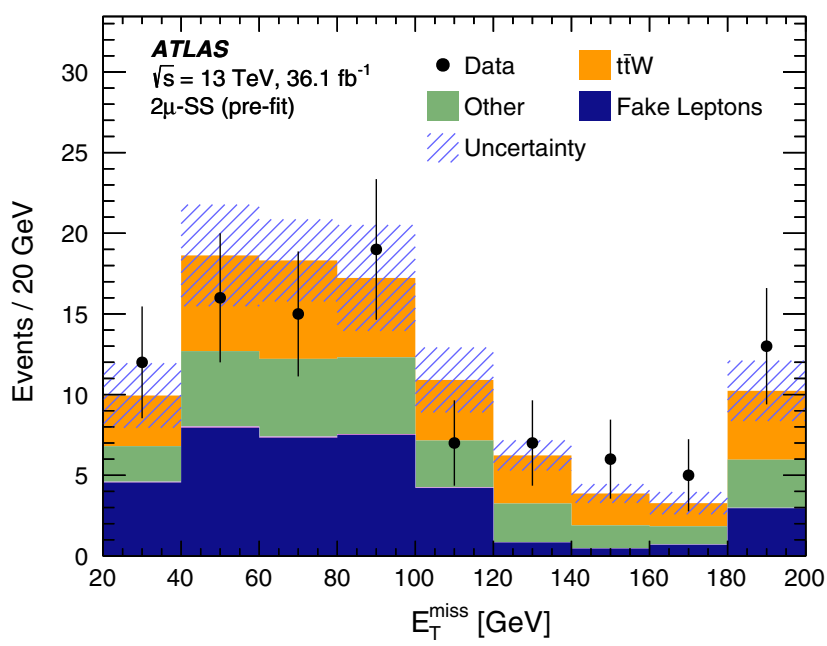

(a)

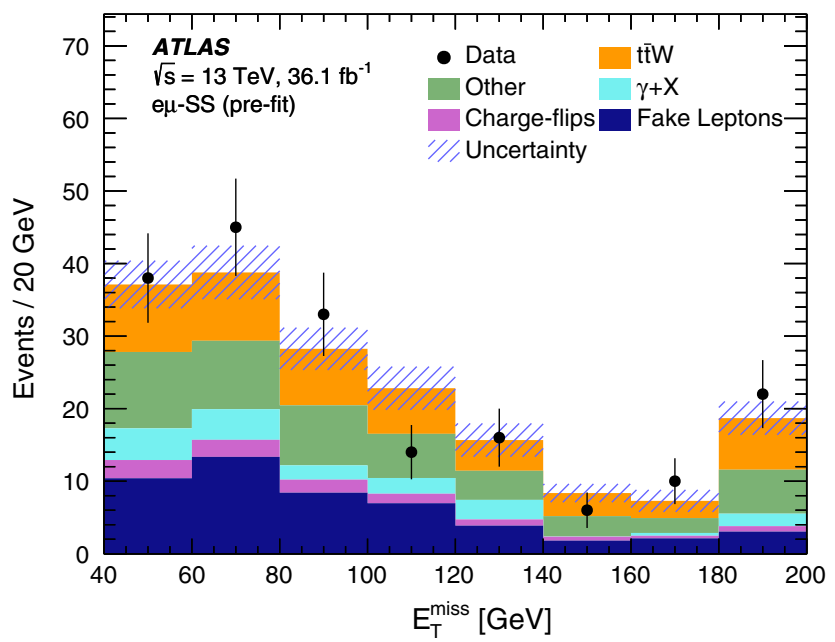

(c)

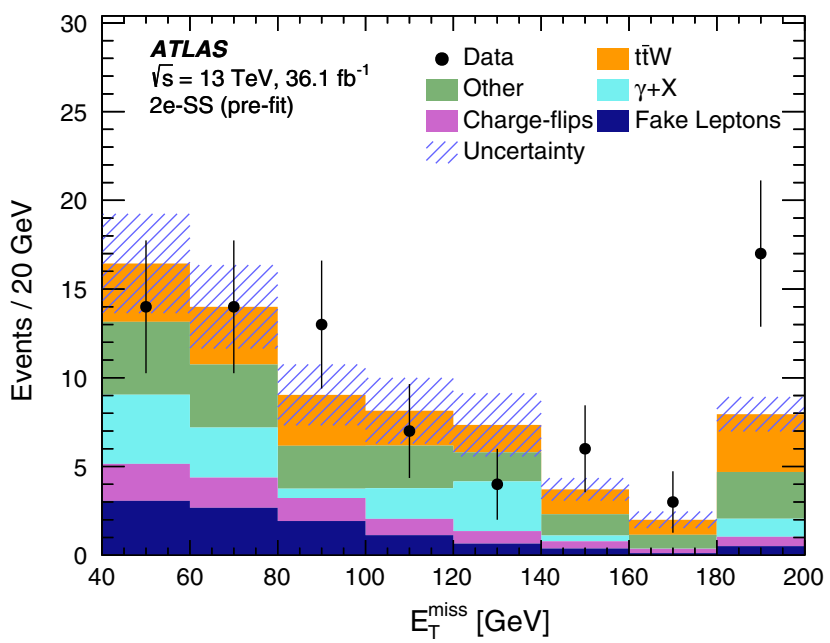

(e)

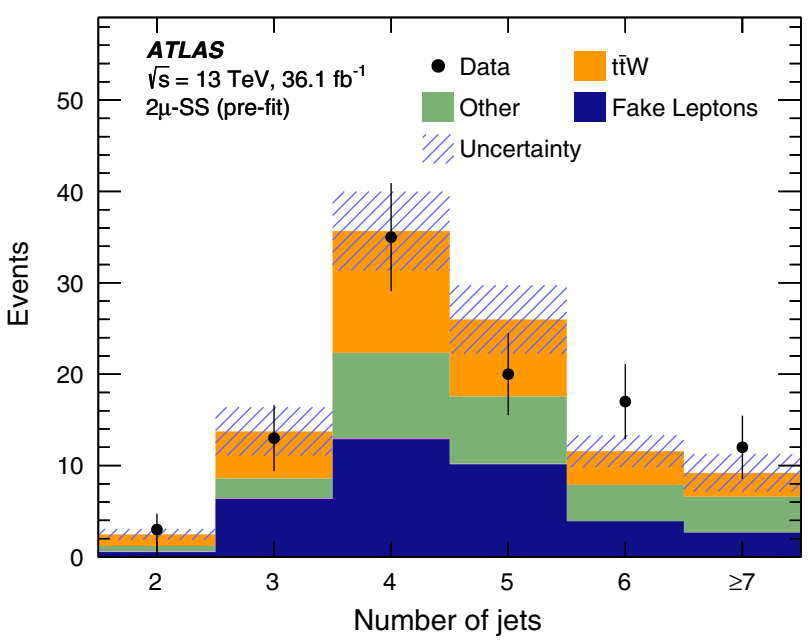

(b)

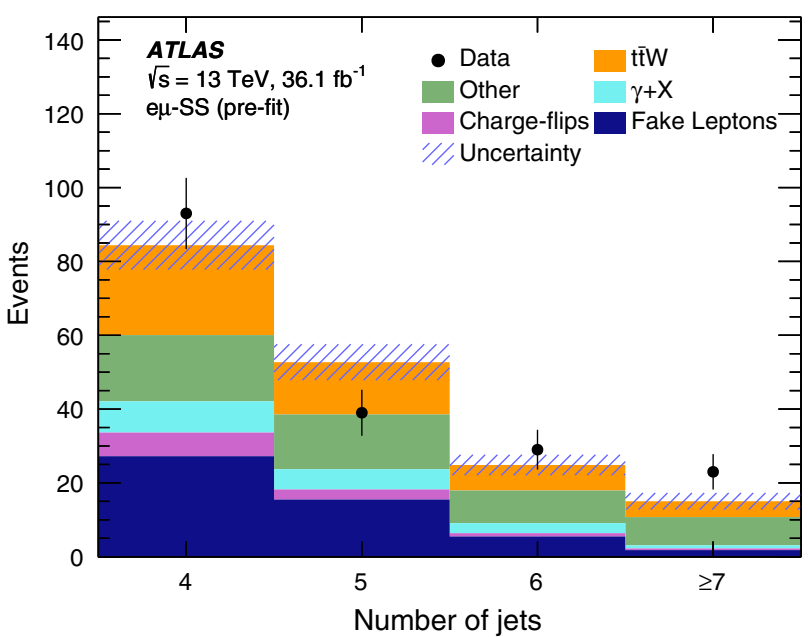

(d)

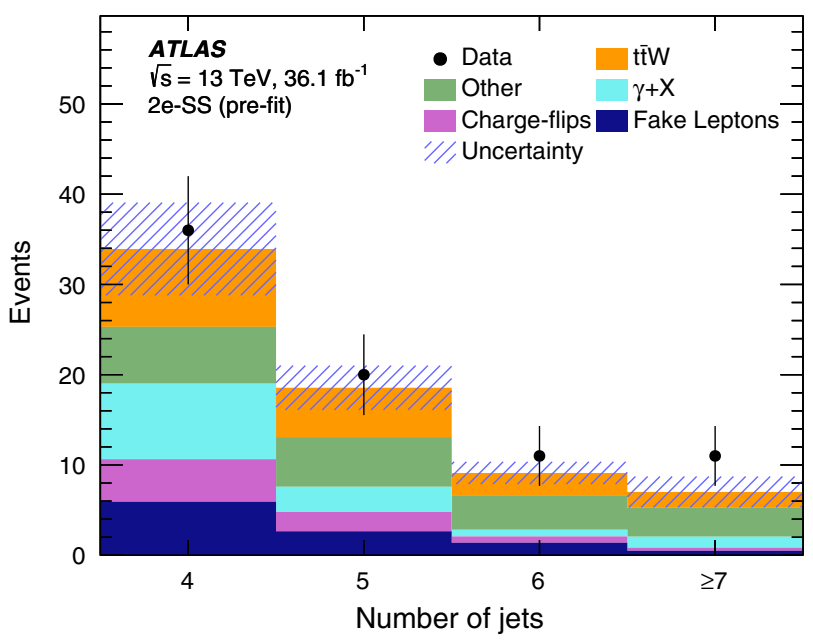

(f)

FIG. 4. Distributions in the $2 \mu$-SS, $e \mu$-SS and $2 e$-SS regions of (a),(c),(e) the missing transverse momentum $E_{\mathrm{T}}^{\text {miss }}$ and (b),(d),(f) the jet multiplicity. The shaded band represents the total uncertainty. The "Other" background contains SM processes with small cross sections producing two same-sign prompt leptons. The last bin in each of the distributions includes the overflow. 
TABLE IV. Summary of event selection requirements in the trilepton signal regions targeting the $t \bar{t} Z$ process.

\begin{tabular}{|c|c|c|c|c|}
\hline Variable & $3 \ell-\mathrm{Z}-1 \mathrm{~b} 4 \mathrm{j}$ & $3 \ell-\mathrm{Z}-2 \mathrm{~b} 3 \mathrm{j}$ & $3 \ell-\mathrm{Z}-2 \mathrm{~b} 4 \mathrm{j}$ & $3 \ell-$ noZ-2b4j \\
\hline Leading lepton & \multicolumn{4}{|c|}{$p_{\mathrm{T}}>27 \mathrm{GeV}$} \\
\hline Other leptons & \multicolumn{4}{|c|}{$p_{\mathrm{T}}>20 \mathrm{GeV}$} \\
\hline Sum of lepton charges & \multicolumn{4}{|c|}{ \pm 1} \\
\hline$Z$ requirement (OSSF pair) & \multicolumn{3}{|c|}{$\left|m_{\ell \ell}-m_{Z}\right|<10 \mathrm{GeV}$} & $\left|m_{\ell \ell}-m_{Z}\right|>10 \mathrm{GeV}$ \\
\hline$n_{\text {jets }}$ & $\geq 4$ & 3 & $\geq 4$ & $\geq 4$ \\
\hline$n_{b \text {-tags }}$ & 1 & $\geq 2$ & $\geq 2$ & $\geq 2$ \\
\hline
\end{tabular}

TABLE V. Summary of event selection requirements in the trilepton signal regions targeting the $t \bar{t} W$ process.

\begin{tabular}{|c|c|c|c|c|}
\hline Variable & $3 \ell \mathrm{p}-\mathrm{noZ}-2 \mathrm{~b} 2 \mathrm{j}$ & $3 \ell \mathrm{m}-\mathrm{noZ}-2 \mathrm{~b} 2 \mathrm{j}$ & $3 \ell \mathrm{p}-\mathrm{noZ}-1 \mathrm{~b} 2 \mathrm{j}$ & $3 \ell \mathrm{m}-\mathrm{noZ}-1 \mathrm{~b} 2 \mathrm{j}$ \\
\hline All leptons & \multicolumn{4}{|c|}{$p_{\mathrm{T}}>27 \mathrm{GeV}$} \\
\hline$Z$ veto (OSSF pair) & \multicolumn{4}{|c|}{$\left|m_{\ell \ell}-m_{Z}\right|>10 \mathrm{GeV}$} \\
\hline$n_{\text {jets }}$ & \multicolumn{4}{|c|}{2 or 3} \\
\hline$H_{\mathrm{T}}$ & \multicolumn{2}{|c|}{$\ldots$} & \multicolumn{2}{|c|}{$>240 \mathrm{GeV}$} \\
\hline Sum of lepton charges & +1 & -1 & +1 & -1 \\
\hline$n_{b \text {-tags }}$ & $\geq 2$ & $\geq 2$ & 1 & 1 \\
\hline
\end{tabular}

regions, the $E_{\mathrm{T}}^{\mathrm{miss}}$ requirement is loosened to $E_{\mathrm{T}}^{\mathrm{miss}}>$ $20 \mathrm{GeV}$, and at least two jets are required. In the $2 e$ and $2 \mu$ signal regions, events containing a pair of leptons whose invariant mass is within $10 \mathrm{GeV}$ of the $Z$ boson mass are vetoed.

The control regions used to measure the fake-lepton efficiencies, as explained at the beginning of this section, are defined to be orthogonal to the SS dilepton signal regions: either the $E_{\mathrm{T}}^{\text {miss }}, H_{\mathrm{T}}$ or jet multiplicity requirements of the signal regions are not satisfied. The $t \bar{t} W$ signal contribution in the control regions where both leptons satisfy the tight criteria is found to be non-

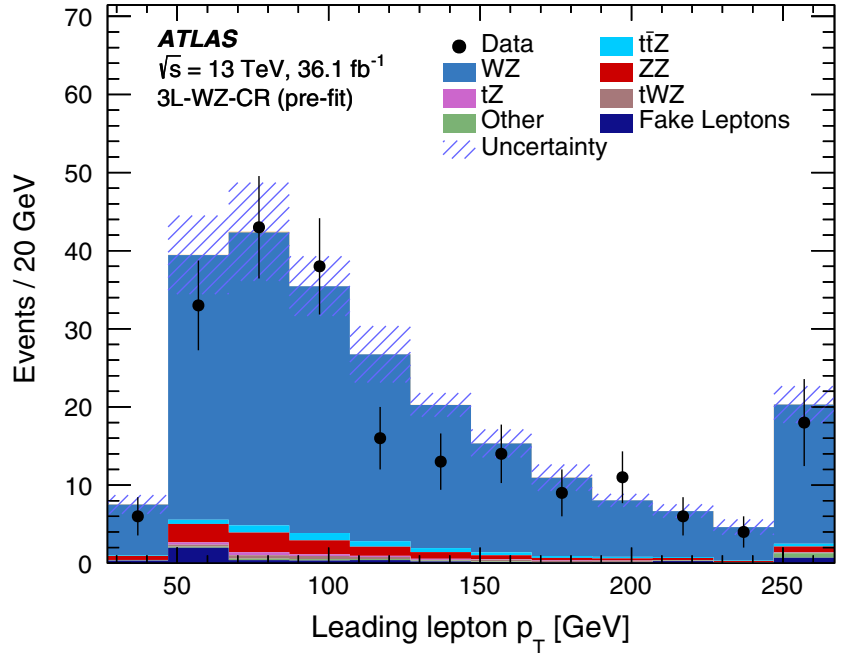

(a) negligible. To enhance the sensitivity of the analysis, the latter regions are also included in the final fit used to measure the $t \bar{t} W$ cross section, as discussed in Sec. VII. These six regions are further split according to the charge of the leptons, and the resulting twelve regions are denoted by $2 \ell-\mathrm{SS}(\mathrm{p}, \mathrm{m})-(1,2) b-\mathrm{CR}$, following the same notation as for the signal regions defined above. In each control region, both leptons are required to have $p_{\mathrm{T}}>27 \mathrm{GeV}$, and at least one (two) jets are required in the $1 b(2 b)$ regions. In addition, events containing a pair of leptons whose invariant mass is compatible with the $Z$ boson mass are vetoed. The largest contamination

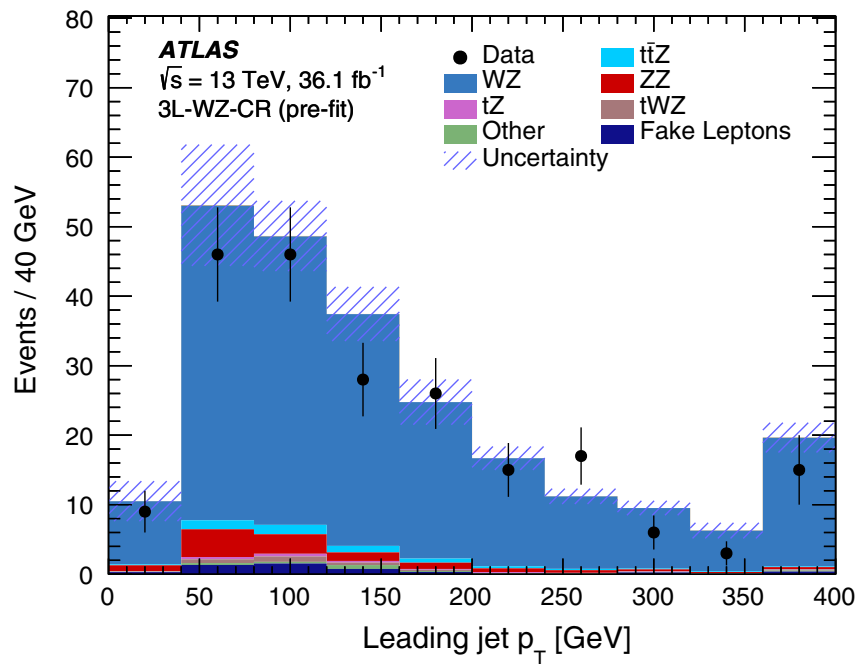

(b)

FIG. 5. Distributions of (a) the leading lepton transverse momentum $p_{\mathrm{T}}$ and (b) the leading jet $p_{\mathrm{T}}$ in the $3 \ell$-WZ-CR control region before the fit. The "Other" background contains SM processes with small cross sections producing three prompt leptons. The shaded band represents the total uncertainty. The last bin in each of the distributions includes the overflow. 
from $t \bar{t} W$ is found to be $25 \%$, in the region $2 \mu$ $\mathrm{SSp} 2 b$-CR.

The dominant background in the $2 \ell$-SS signal regions arises from events containing fake leptons. Backgrounds from the production of prompt leptons with correctly identified charge come primarily from $t \bar{t} H$ and $W Z$ production. The charge-flip background is also significant in signal regions with two electrons. In regions with two muons, this background is negligible as the probability of misidentifying the charge of a muon in the relevant $p_{\mathrm{T}}$ range is very small. To validate the charge-flip background, a validation region called $2 e-\mathrm{SS}-1 \mathrm{~b}-\mathrm{VR}$ is constructed similarly to the $2 e-\mathrm{SS}$ signal regions, except that the number of jets is required to be between one and three, to ensure orthogonality with the signal regions. The requirement on $H_{\mathrm{T}}$ is also removed, exactly one jet is required to be $b$-tagged, and the invariant mass of the lepton pair is required to be greater than $15 \mathrm{GeV}$. The distributions of $m_{\ell \ell}$ and the leading lepton $p_{\mathrm{T}}$ are shown in Fig. 2, demonstrating good modeling of the charge-flip background.

Figure 3 shows the distributions of $H_{\mathrm{T}}, E_{\mathrm{T}}^{\text {miss }}$ and the subleading lepton $p_{\mathrm{T}}$, for the control regions $2 e$-SS- $2 b$-CR, $e \mu$-SS- $1 b$-CR and $2 \mu$-SS- $1 b$-CR. The data and the expectation agree well, demonstrating the validity of the description of the fake-lepton background determined by the matrix method.

To facilitate comparisons of data with the expectation, three regions $2 e$-SS, $e \mu$-SS and $2 \mu$-SS are formed by combining all the same-sign signal regions corresponding to a given lepton flavor combination. The distributions of $E_{\mathrm{T}}^{\mathrm{miss}}$ and the number of jets for these three regions are shown in Fig. 4.

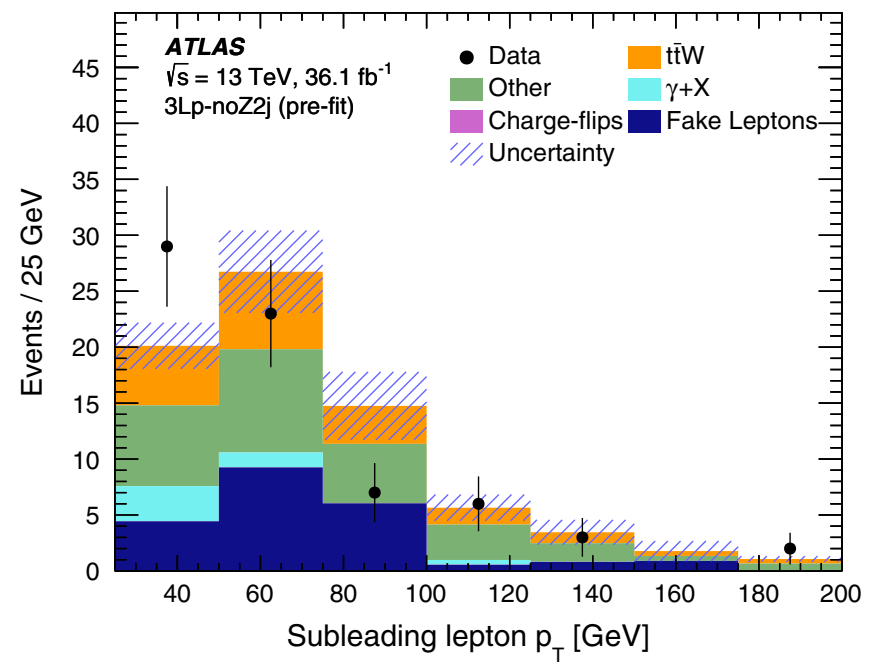

(a)

\section{Trilepton analysis}

Eight signal regions with exactly three leptons are considered, four of them targeting the $\bar{t} \bar{Z}$ and four targeting the $t \bar{t} W$ process, as defined in Tables IV and V, respectively. The regions are divided into two groups depending on whether or not a pair of OSSF leptons with invariant mass within $10 \mathrm{GeV}$ of the $Z$ boson mass is present. The signal regions are further categorized according to jet and $b$-tagged jet multiplicities.

The four signal regions in the first group are sensitive to $t \bar{t} Z$. In the $3 \ell-Z-1 \mathrm{~b} 4 \mathrm{j}$ region, at least four jets are required, exactly one of which is $b$-tagged. In the $3 \ell-Z-2 b 3 \mathrm{j}$ region, exactly three jets with at least two $b$-tagged jets are required. In the $3 \ell-\mathrm{Z}-2 \mathrm{~b} 4 \mathrm{j}$ region, at least four jets are required, of which at least two are $b$-tagged. In the $3 \ell$-noZ$2 \mathrm{~b} 4 \mathrm{j}$ region, targeting events with an off-shell $Z^{*}$ or $\gamma^{*}$, at least four jets are required, of which at least two are $b$-tagged; no OSSF lepton pair is allowed in the $Z$ boson mass window. The sum of the lepton charges must be \pm 1 .

The remaining four trilepton signal regions target the $t \bar{t} W$ process. These regions require two or three jets and veto events that contain an OSSF pair of leptons whose invariant mass is within $10 \mathrm{GeV}$ of the $Z$ boson mass. In the first two regions, $3 \ell \mathrm{p}$-noZ- $2 \mathrm{~b} 2 \mathrm{j}$ and $3 \ell \mathrm{m}$-noZ-2b $2 \mathrm{j}$, at least two jets are required to be $b$-tagged. In the other two regions, $3 \ell \mathrm{p}-\mathrm{noZ}-1 \mathrm{~b} 2 \mathrm{j}$ and $3 \ell \mathrm{m}-\mathrm{noZ}-1 \mathrm{~b} 2 \mathrm{j}$, exactly one jet is required to be $b$-tagged. The sum of lepton charges is required to be $+1(-1)$ in $3 \ell \mathrm{p}-\mathrm{noZ}-2 \mathrm{~b} 2 \mathrm{j}$ and $3 \ell \mathrm{p}-\mathrm{noZ}-1 \mathrm{~b} 2 \mathrm{j}$ $(3 \ell \mathrm{m}-\mathrm{noZ}-2 \mathrm{~b} 2 \mathrm{j}$ and $3 \ell \mathrm{m}-\mathrm{noZ}-1 \mathrm{~b} 2 \mathrm{j})$. In regions $3 \ell \mathrm{p}-\mathrm{noZ}-$ $1 \mathrm{~b} 2 \mathrm{j}$ and $3 \ell \mathrm{m}-\mathrm{noZ}-1 \mathrm{~b} 2 \mathrm{j}, H_{\mathrm{T}}>240 \mathrm{GeV}$ is also required. The signal region definitions for the trilepton channel are

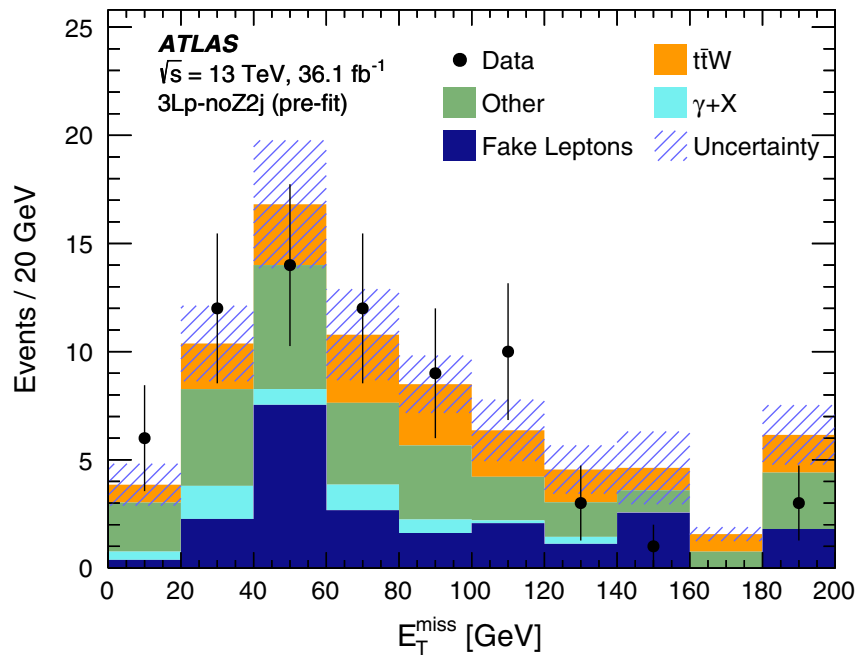

(b)

FIG. 6. Distributions of (a) the subleading lepton transverse momentum $p_{\mathrm{T}}$ and (b) missing transverse momentum $E_{\mathrm{T}}^{\mathrm{miss}}$ for events belonging to any of the four trilepton regions targeting the $t \bar{t} W$ process. The distributions are shown before the fit. The "Other" background contains SM processes with small cross sections producing three prompt leptons. The shaded band represents the total uncertainty. The last bin in each of the distributions includes the overflow. 


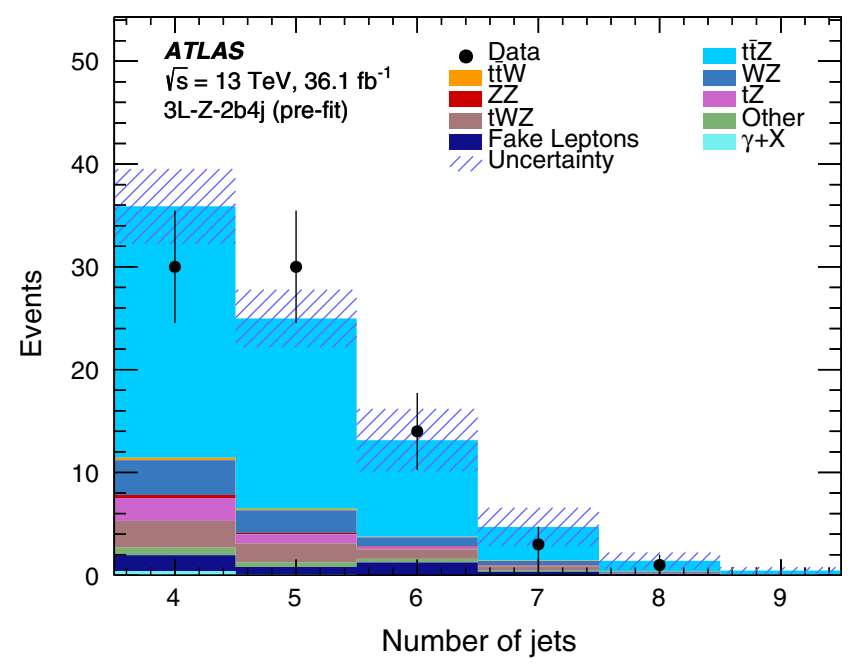

(a)

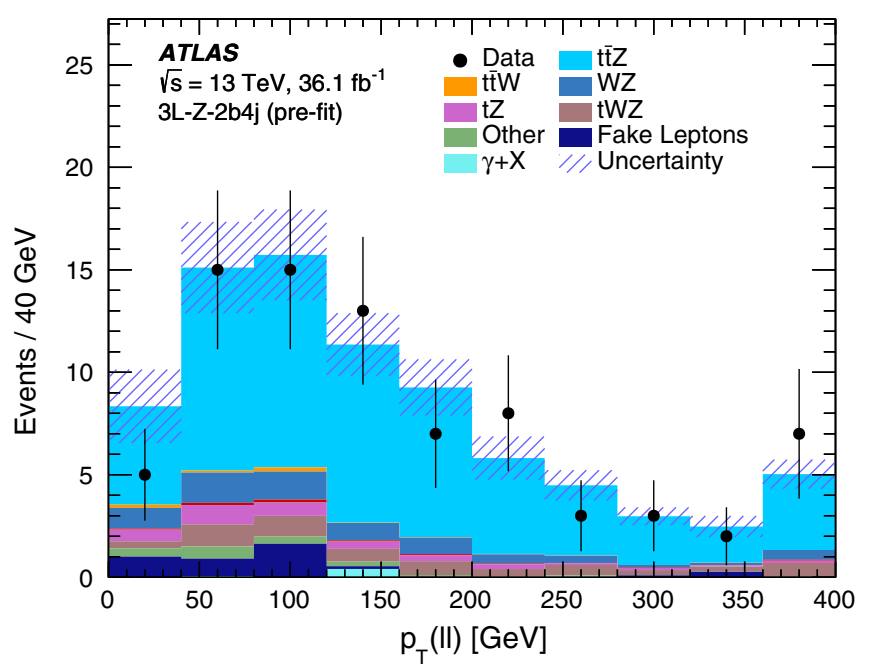

(b)

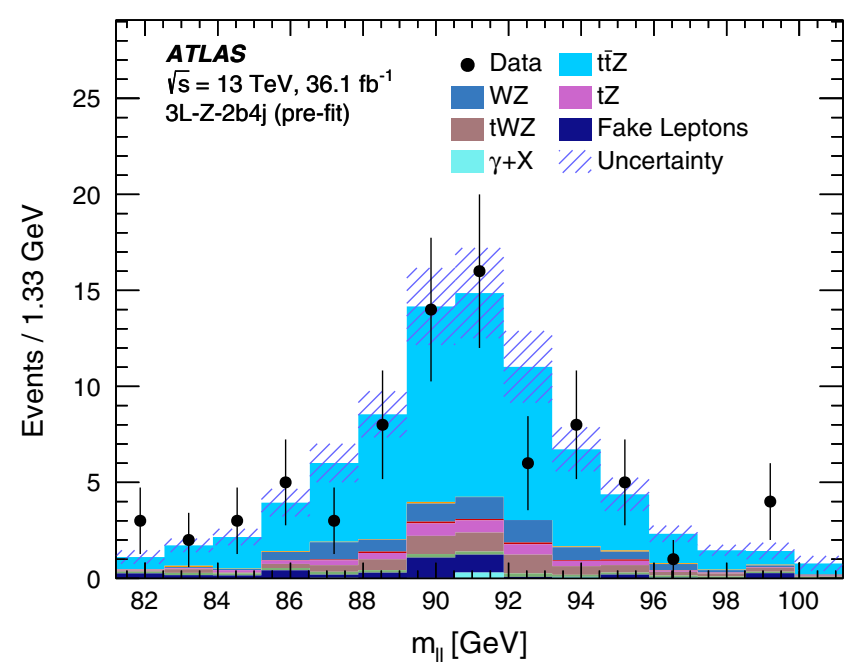

(c)

FIG. 7. Distributions of (a) the number of jets, (b) the transverse momentum $p_{\mathrm{T}}\left(\ell \ell\right.$ ) and (c) the mass $m_{\ell \ell}$ of the reconstructed $Z$ boson candidate for events in $3 \ell-\mathrm{Z}-2 \mathrm{~b} 4 \mathrm{j}$. The distributions are shown before the fit. The "Other" background contains SM processes with small cross sections producing three prompt leptons. The shaded band represents the total uncertainty. The last bin in each of the distributions includes the overflow.

summarized in Tables IV and $\mathrm{V}$ for the signal regions targeting $t \bar{t} Z$ and $t \bar{t} W$, respectively.

The dominant backgrounds in the $3 \ell-Z-1 \mathrm{~b} 4 \mathrm{j}, 3 \ell-Z-2 \mathrm{~b} 3 \mathrm{j}$ and $3 \ell-Z-2 \mathrm{~b} 4 \mathrm{j}$ signal regions arise from diboson production, the production of a single top quark in association with a $Z$ boson ( $t Z$ and $t W Z$ ) and $Z+$ jets production with a fake lepton.

A control region is used to determine the normalization of the $W Z+$ jets background in data. Exactly three leptons are required, at least one pair of which must be an OSSF

TABLE VI. Definitions of the four signal regions in the tetralepton channel.

\begin{tabular}{|c|c|c|c|c|c|c|}
\hline Region & $Z_{2}$ leptons & $p_{\mathrm{T} 4}$ & $p_{\mathrm{T} 34}$ & $\left|m_{Z_{2}}-m_{Z}\right|$ & $E_{\mathrm{T}}^{\text {miss }}$ & $n_{b \text {-tags }}$ \\
\hline $4 \ell-\mathrm{DF}-1 \mathrm{~b}$ & $e^{ \pm} \mu^{\mp}$ & $\ldots$ & $>35 \mathrm{GeV}$ & $\ldots$ & $\ldots$ & 1 \\
\hline $4 \ell-\mathrm{DF}-2 \mathrm{~b}$ & $e^{ \pm} \mu^{\mp}$ & $>10 \mathrm{GeV}$ & $\cdots$ & $\ldots$ & $\ldots$ & $\geq 2$ \\
\hline $4 \ell-\mathrm{SF}-1 \mathrm{~b}$ & $e^{ \pm} e^{\mp}, \mu^{ \pm} \mu^{\mp}$ & $\cdots$ & $>25 \mathrm{GeV}$ & $\begin{array}{l}>10 \mathrm{GeV} \\
<10 \mathrm{GeV}\end{array}$ & $\left.\begin{array}{l}>40 \mathrm{GeV} \\
>80 \mathrm{GeV}\end{array}\right\}$ & 1 \\
\hline $4 \ell-\mathrm{SF}-2 \mathrm{~b}$ & $e^{ \pm} e^{\mp}, \mu^{ \pm} \mu^{\mp}$ & $>10 \mathrm{GeV}$ & $\cdots$ & $\begin{array}{l}>10 \mathrm{GeV} \\
<10 \mathrm{GeV}\end{array}$ & 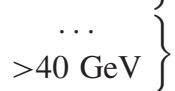 & $\geq 2$ \\
\hline
\end{tabular}


pair with an invariant mass within $10 \mathrm{GeV}$ of the $Z$ boson mass. There must be exactly three jets, none of which pass the $b$-tagging requirement. This region is referred to as $3 \ell$-WZ-CR and it is included in the fit. Distributions comparing data with SM predictions in $3 \ell$-WZ-CR are shown in Fig. 5, demonstrating good modeling of the $W Z$ background.

Figure 6 shows the leading lepton $p_{\mathrm{T}}$ and $E_{\mathrm{T}}^{\mathrm{miss}}$ for events belonging to any of the four trilepton regions targeting $t \bar{t} W$. Distributions of the number of jets, the $p_{\mathrm{T}}$ and mass of the reconstructed $Z$ boson candidate in the signal region most sensitive to $t \bar{t} Z, 3 \ell-Z-2 \mathrm{~b} 4 \mathrm{j}$, are shown in Fig. 7.

\section{Tetralepton analysis}

The tetralepton channel targets the $t \bar{t} Z$ process for the case where both $W$ bosons, resulting from top-quark decays, and the $Z$ boson decay leptonically. Events with two pairs of opposite-sign leptons are selected, and at least one pair must have the same flavor. The OSSF lepton pair with reconstructed invariant mass closest to $m_{Z}$ is attributed to the $Z$ boson decay and denoted in the following by $Z_{1}$. The two remaining leptons are used to define $Z_{2}$. The signal region definitions for the tetralepton channel are summarized in Table VI. Four signal regions are defined according to the relative flavor of the two $Z_{2}$ leptons, different flavor (DF) or same flavor (SF), and the number of $b$-tagged jets: one, or at least two $(1 b, 2 b)$. The signal regions are thus $4 \ell$-DF- $1 \mathrm{~b}, 4 \ell$-DF- $2 \mathrm{~b}, 4 \ell$-SF- $1 \mathrm{~b}$ and $4 \ell$-SF- $2 \mathrm{~b}$.

In the same-flavor regions, requirements on $E_{\mathrm{T}}^{\text {miss }}$ are applied to suppress the $Z Z$ background. In the $4 \ell-\mathrm{SF}-1 \mathrm{~b}$ signal region, the $E_{\mathrm{T}}^{\text {miss }}$ is required to be greater than $80 \mathrm{GeV}(40 \mathrm{GeV})$ for events with $\left|m_{Z_{2}}-m_{Z}\right|<10 \mathrm{GeV}$ $\left(\left|m_{Z_{2}}-m_{Z}\right|>10 \mathrm{GeV}\right)$. In the $4 \ell$-SF-2b signal region,

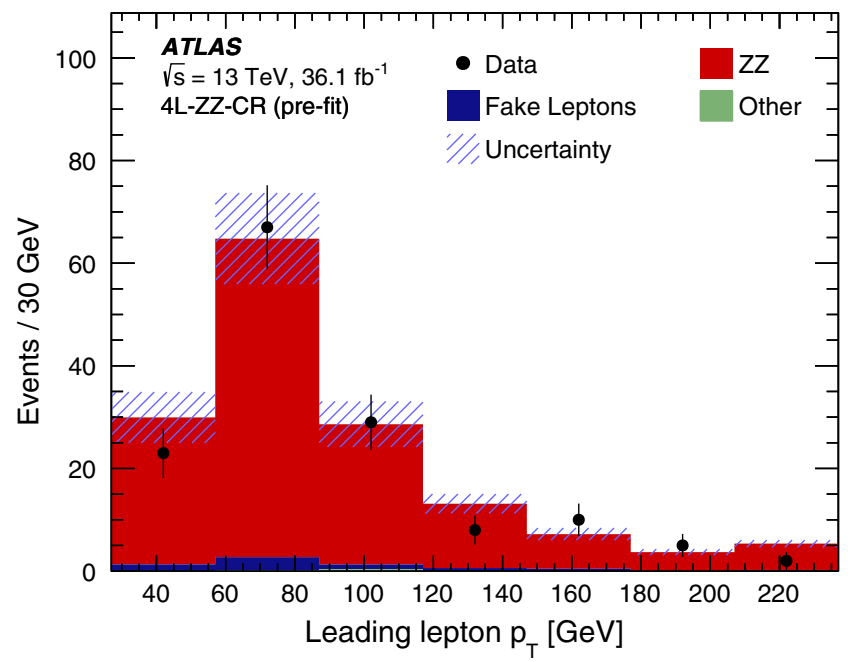

(a) a requirement of $E_{\mathrm{T}}^{\text {miss }}>40 \mathrm{GeV}$ is applied for events with $\left|m_{Z_{2}}-m_{Z}\right|<10 \mathrm{GeV}$.

To suppress events with fake leptons in the 1-b-tag multiplicity regions, additional requirements on the scalar sum of the transverse momenta of the third and fourth leptons $\left(p_{\mathrm{T} 34}\right)$ are imposed. In the $4 \ell$-SF- $1 \mathrm{~b}$ and $4 \ell$-DF- $1 \mathrm{~b}$ regions, events are required to satisfy $p_{\mathrm{T} 34}>25 \mathrm{GeV}$ and $p_{\mathrm{T} 34}>35 \mathrm{GeV}$, respectively, while in the other regions all leptons are required to satisfy $p_{\mathrm{T}}>10 \mathrm{GeV}$.

A control region used to determine the $Z Z$ normalization, referred to as $4 \ell$-ZZ-CR, is included in the fit and is defined to have exactly four reconstructed leptons, a $Z_{2}$ pair with OSSF leptons, the value of both $m_{Z_{1}}$ and $m_{Z_{2}}$ within $10 \mathrm{GeV}$ of the mass of the $Z$ boson, and $20 \mathrm{GeV}<E_{\mathrm{T}}^{\text {miss }}<$ $40 \mathrm{GeV}$. The leading lepton $p_{\mathrm{T}}$ and the jet multiplicity in this control region are shown in Fig. 8, and good agreement is seen between data and prediction.

The contribution from backgrounds containing fake leptons is estimated from simulation and corrected with scale factors determined in two control regions: one region enriched in $t \bar{t}$ events and one region enriched in $Z+$ jets events. The scale factors are extracted and applied separately for electron and muon fake-lepton candidates, and for leptons arising from heavy-flavor hadrons and other sources. Therefore, a total of four scale factors are determined. The scale factors are applied to all $\mathrm{MC}$ simulation events with fewer than four prompt leptons according to the number, flavor and origin of the fake leptons. It is verified that the scale factors for different generators used in the simulation are consistent with each other.

Figure 9 compares the data with the expected distributions for all four signal regions combined, showing good agreement between data and expectation.

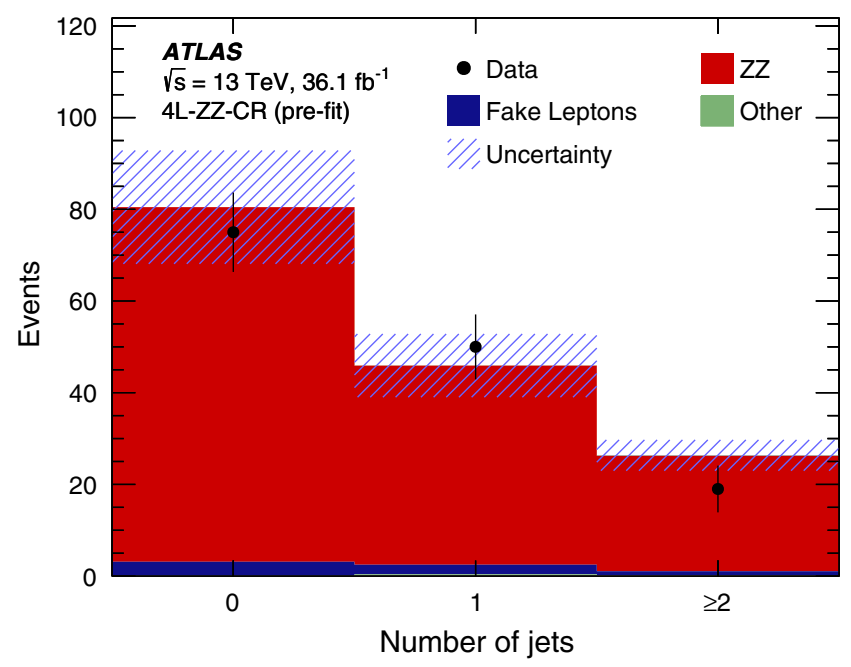

(b)

FIG. 8. Distribution of (a) the leading lepton transverse momentum $p_{\mathrm{T}}$ and (b) jet multiplicity in the $4 \ell$-ZZ-CR control region. The distributions are shown before the fit. The shaded band represents the total uncertainty. The last bin in each of the distributions includes the overflow. 


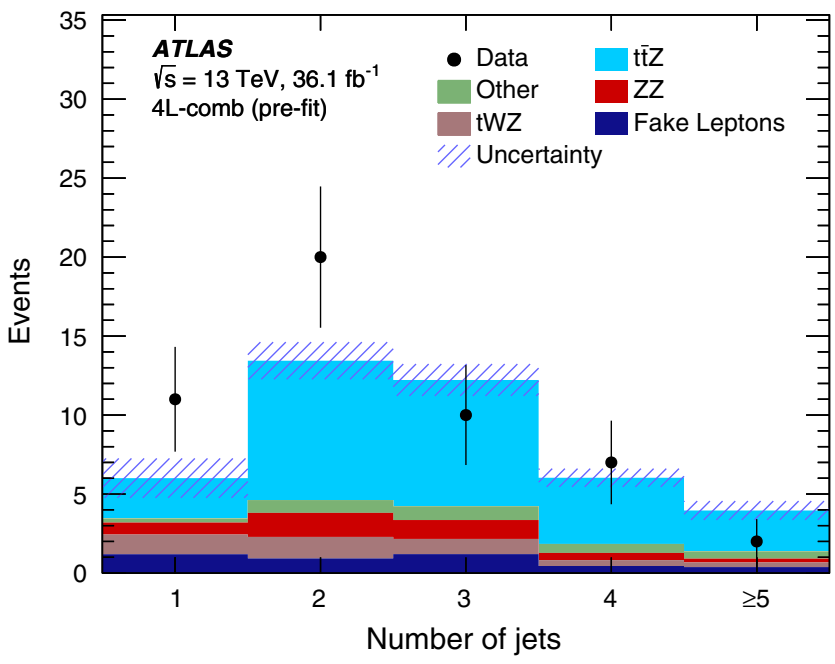

(a)

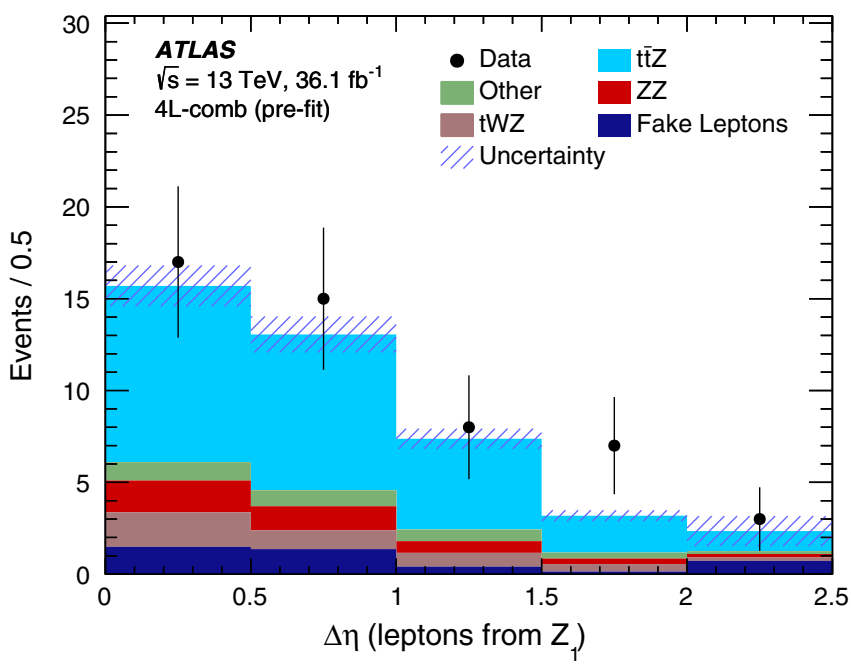

(c)

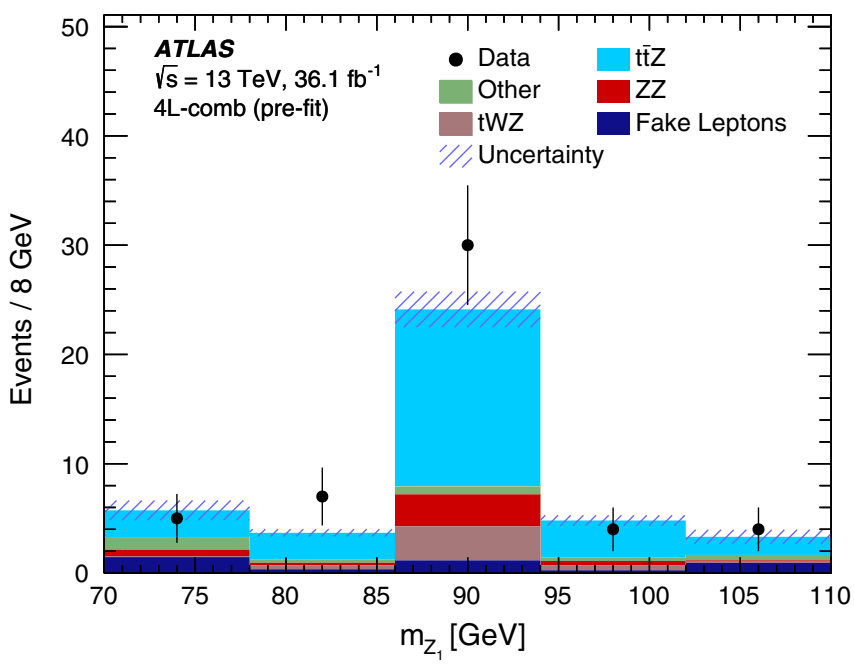

(b)

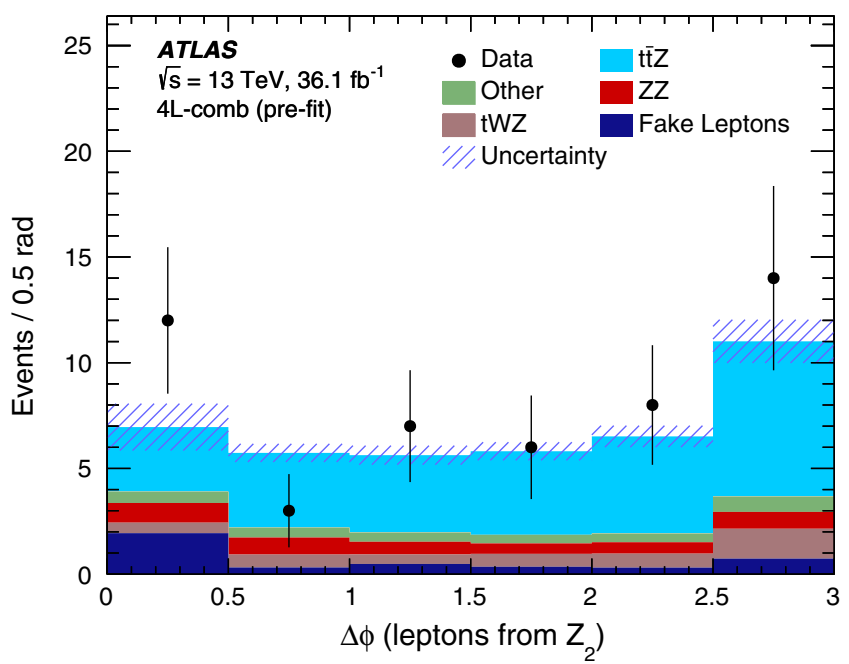

(d)

FIG. 9. Distributions, for all tetralepton signal regions combined, of (a) the number of jets, (b) the invariant mass of the OSSF lepton pair closest to the $Z$ boson mass, $m_{Z_{1}}$, (c) the pseudorapidity separation $\Delta \eta$ for that pair of leptons and (d) the azimuthal angle $\Delta \phi$ between the remaining two leptons. The "Other" background contains SM processes with small cross sections producing four prompt leptons. The distributions are shown before the fit. The shaded band represents the total uncertainty. The first and last bin include the underflow and overflow, respectively.

\section{SYSTEMATIC UNCERTAINTIES}

The signal and background yields in each signal and control region may be affected by several sources of systematic uncertainty. These are implemented as nuisance parameters in the fit, explained in Sec. VII, are constrained by Gaussian probability density functions and are described in the following subsections.

\section{A. Luminosity}

The uncertainty in the integrated luminosity of the dataset is $2.1 \%$. It is derived, following a methodology similar to that detailed in Ref. [23], and using the LUCID-2 detector for the baseline luminosity measurements [24], from a calibration of the luminosity scale using $x-y$ beamseparation scans. This systematic uncertainty affects all processes modeled using Monte Carlo simulations, apart from $Z+1 \mathrm{HF}, Z+2 \mathrm{HF}, W Z$ and $Z Z$, whose normalizations are taken from data control regions.

\section{B. Uncertainties associated with reconstructed objects}

Uncertainties associated with the lepton selection arise from the trigger, reconstruction, identification and isolation efficiencies, and lepton momentum scale and resolution [59,71-73].

Uncertainties associated with the jet selection arise from the jet energy scale (JES), the JVT requirement and the jet 
energy resolution (JER). The JES and its uncertainty are derived by combining information from test-beam data, collision data and simulation [64]. The uncertainties in the JER and JVT have a significant effect at low jet $p_{\mathrm{T}}$.

The efficiency of the flavor-tagging algorithm is measured for each jet flavor using control samples in data and in simulation. From these measurements, correction factors are derived to correct the tagging rates in the simulation. In the case of $b$-jets, correction factors and their uncertainties are estimated from data using dileptonic $t \bar{t}$ events [66]. In the case of $c$-jets, they are derived using jets from $W$ boson decays in $t \bar{t}$ events [74]. In the case of light-flavor jets, correction factors are derived using dijet events [75]. Sources of uncertainty affecting the $b$ - and $c$-tagging efficiencies are considered as a function of jet $p_{\mathrm{T}}$, including bin-to-bin correlations [76]. The uncertainty in the efficiency for tagging light-flavor jets depends on the jet $p_{\mathrm{T}}$ and on $\eta$. These systematic uncertainties are taken as uncorrelated between $b$-jets, $c$-jets, and light-flavor jets. An additional uncertainty is assigned to account for the extrapolation of the $b$-tagging efficiency measurement from the $p_{\mathrm{T}}$ region used to determine the correction factors to regions with higher transverse momentum.

The treatment of the uncertainties associated with reconstructed objects is common to all analysis channels, and thus these are considered as fully correlated among different analysis regions.

\section{Uncertainties in the signal modeling}

Four sources of systematic uncertainties in the theoretical predictions of the $t \bar{t} Z$ and $t \bar{t} W$ processes are considered. These signal modeling uncertainties are treated as uncorrelated between the two processes, and correlated among channels. Taking instead the uncertainties as correlated between the two processes has a negligible impact on the results. Acceptance effects due to the choice of scale and PDF in the nominal MG5_aMC+PYTHIA 8 (A14 tune) sample are considered. The renormalization and factorization scales $\mu_{\mathrm{R}}=\mu_{\mathrm{F}}$ are varied simultaneously by factors 2.0 and 0.5. In addition, the effects of a set of variations in the tune parameters (A14 eigentune variations), sensitive to initial- and final-state radiation, multiple parton interactions and color reconnection, are evaluated [36]. Studies performed at particle level show that the largest impact comes from variations in initial-state radiation [77]. The systematic uncertainty due to the choice of generator for the $t \bar{t} Z$ and $t \bar{t} W$ acceptance is estimated by comparing the nominal sample with one generated with SHERPA v2.2. The SHERPA sample uses the LO matrix element with up to one (two) additional parton(s) included in the matrix element calculation for $t \bar{t} Z(t \bar{t} W)$ and merged with the SHERPA parton shower [43] using the ME+PS@LO prescription. The NNPDF3 . ONLO PDF set is used in conjunction with a dedicated parton-shower tune developed by the SHERPA authors.

\section{Uncertainties in the background modeling}

The $Z+$ jets process is, together with $t \bar{t}$ production, the dominant background in the OS dilepton channel. Its normalization is extracted from data as described in Sec. VA, but the shape of the BDT distribution is obtained from simulation. To assess the systematic uncertainty in the shape, the renormalization, factorization and resummation scales used in the MC generation are varied by a factor of two with respect to the nominal values.

The normalization and shape of the $t \bar{t}$ background in the OS dilepton channel is obtained using the data-driven method detailed in Sec. VA. A systematic uncertainty arises from the factor used to obtain $t \bar{t}$ background yields in the same-flavor signal regions from corresponding opposite-flavor dilepton control regions. The uncertainty is due to the finite size of the samples of simulated events used, and the difference between the values of the factor obtained with the nominal PowHEG-Box+PythiA 8 sample and an alternative sample generated using MG5_aMC+PythIA 8. The total uncertainty in the factor is found to be $3 \%$.

In the trilepton regions sensitive to $t \bar{t} Z$, the normalization of the $W Z$ background is treated as a free parameter in the fit used to extract the $t \bar{t} Z$ and $t \bar{t} W$ signals. The uncertainty in the extrapolation of the $W Z$ background estimate from the control region to signal regions with specific jet and $b$-tag multiplicities is evaluated by comparing predictions obtained by varying the renormalization, factorization and resummation scales used in $\mathrm{MC}$ generation. The uncertainties vary from $30 \%$ to $50 \%$, depending on the signal region.

The normalization of the $Z Z$ background is treated as a free parameter in the fit. An additional uncertainty arises from the extrapolation from the $4 \ell$-ZZ-CR control region to the signal regions. It is assessed by varying the renormalization, factorization and resummation scales used in $\mathrm{MC}$ generation, and found to be in the range $20 \%-40 \%$.

The uncertainty in the $t \bar{t} H$ background is evaluated by varying the factorization and renormalization scales up and down by a factor of two with respect to the nominal values. It is found to be around $10 \%$.

An overall normalization uncertainty of $30 \%$ is assigned to the $t Z$ background, motivated by the measurements of this process presented in Refs. [78,79]. An additional uncertainty affecting the distribution of this background as a function of jet and $b$-tagged jet multiplicities is evaluated by varying the factorization and renormalization scales, as well as the amount of radiation in the Perugia2 012 parton-shower tune.

An uncertainty of $10 \%$ is assigned to the $t W Z$ background cross section, resulting from different prescriptions for removing the interference with the $t \bar{t} Z$ process. The shape uncertainty is evaluated by varying the factorization and renormalization scales up and down by a factor of two with respect to the nominal value. 
For other prompt-lepton backgrounds, uncertainties of $20 \%$ are assigned to the normalizations of the $\mathrm{WH}$ and $\mathrm{ZH}$ processes, based on calculations from Ref. [34]. An uncertainty of $50 \%$ is considered for triboson and samesign $W W$ processes.

A $10 \%$ uncertainty is applied to the charge-flip background, resulting from uncertainties in the charge-flip rates extracted from a control sample as described in Sec. V.

A 30\% uncertainty is assigned to the contribution from events with two prompt leptons or one prompt lepton and a photon conversion in the control regions used to measure the fake-lepton efficiency. In the SS dilepton channel regions and trilepton regions targeting $t \bar{t} W$, there are 22 nuisance parameters corresponding to the statistical uncertainty in the measurement of the fake-lepton efficiencies. One nuisance parameter is used for each $p_{\mathrm{T}}$ bin used in the

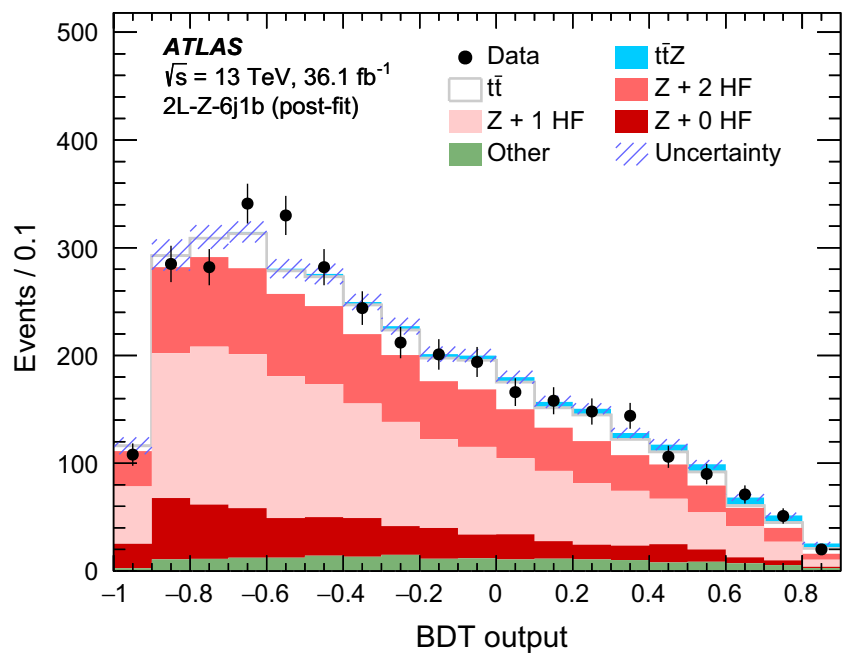

(a) measurement of the fake-lepton efficiencies. For fake-lepton efficiencies in events with one (at least two) $b$-tagged jet(s), seven (four) bins are used, and there is one nuisance parameter for each of the two lepton flavors. In the trilepton signal regions targeting $t \bar{t} Z$, where the fake-lepton background is less important, a simplified description of the fake-lepton uncertainties is used, with one nuisance parameter for each of the two lepton flavors. These nuisance parameters correspond to the maximum of the up and down shifts of the fake-lepton efficiencies resulting from statistical uncertainties and the prompt lepton background subtraction in the control regions used to measure the fake-lepton efficiency. The uncertainties in the fake-lepton background in the $t \bar{t} Z$ and $t \bar{t} W$ analysis regions are considered to be uncorrelated, due to the different lepton selection requirements used in the two sets of regions.

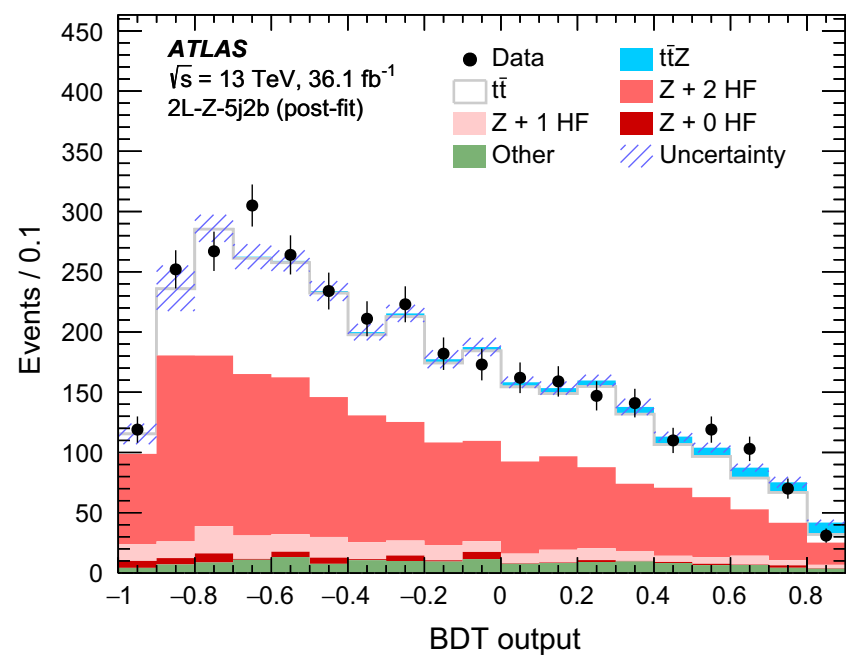

(b)

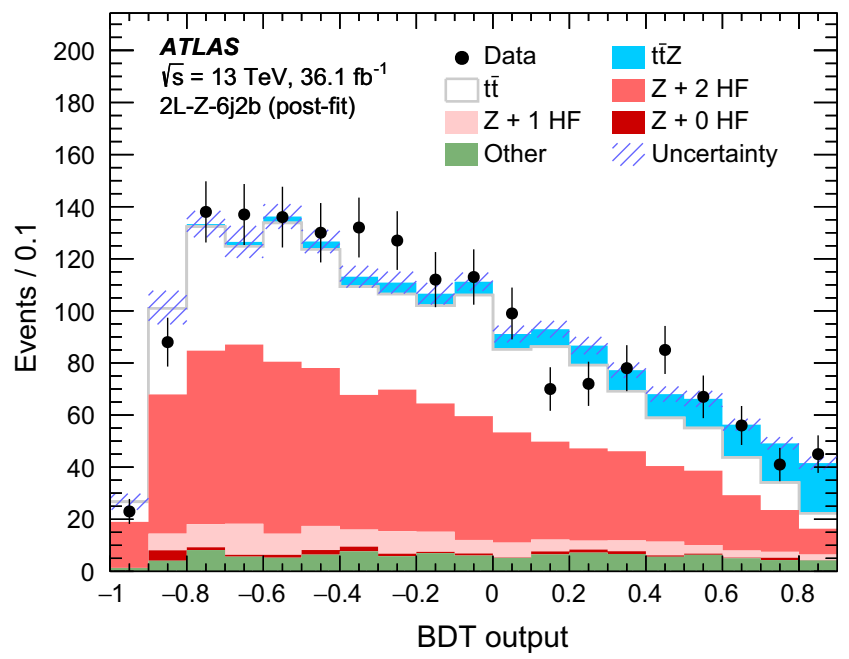

(c)

FIG. 10. The BDT distributions for the OS dilepton signal regions, (a) $2 \ell-Z-6 \mathrm{j} 1 \mathrm{~b}$, (b) $2 \ell-Z-5 \mathrm{j} 2 \mathrm{~b}$, (c) $2 \ell-Z-6 \mathrm{j} 2 \mathrm{~b}$. The distributions are shown after the fit. The "Other" background contains SM processes with small cross sections producing two opposite-sign prompt leptons. The shaded band represents the total uncertainty. The last bin of each distribution contains the overflow. 
Uncertainties in scale factors applied to the fake-lepton background are taken into account in the fake-lepton background yield in the tetralepton channel and the $t \bar{t} \gamma$ background contribution in the trilepton and $2 \ell$-SS channels. These uncertainties are associated with reconstructed objects and the limited sizes of control regions in which the scale factors are obtained. The scale factors have uncertainties between $10 \%$ and $50 \%$, depending on the fake-lepton flavor and source. The $2 \ell$-SS and trilepton fake-lepton systematic uncertainties from the matrix method are assumed to be uncorrelated with the systematic uncertainties in the fake-lepton scale factors.

\section{RESULTS}

The signal strengths $\mu_{\bar{t} \bar{Z} Z}$ and $\mu_{t \bar{t} W}$, defined as the ratios of the measured values of the inclusive production cross sections to the corresponding SM predictions discussed in Sec. III, are extracted simultaneously using a binned maximum-likelihood fit to the numbers of events in the dilepton, trilepton and tetralepton signal and control regions. In the OS dilepton channel signal regions $2 \ell$-Z$6 \mathrm{j} 1 \mathrm{~b}, 2 \ell-\mathrm{Z}-5 \mathrm{j} 2 \mathrm{~b}$ and $2 \ell-\mathrm{Z}-6 \mathrm{j} 2 \mathrm{~b}$, the BDT output distribution is fitted. In the SS dilepton channel, the twelve signal regions $2 \ell$-SSp- $1 b, 2 \ell$-SSm- $1 b, 2 \ell$-SSp- $2 b$ and $2 \ell$-SSm$2 b$ are fitted together with the twelve control regions $2 \ell$-SSp- $1 b$-CR, $\quad 2 \ell$-SSm- $1 b$-CR, $\quad 2 \ell$-SSp- $2 b$-CR and $2 \ell$-SSm- $2 b$-CR defined in Sec. V. The contribution from the $t \bar{t} W$ signal in the SS dilepton control regions is taken into account in the fit. The dependence of the fake-lepton background in these regions on the $t \bar{t} W$ signal strength is also taken into account. In the trilepton channel, the eight signal regions described in Sec. V are included in the fit, as is the $3 \ell$-WZ-CR control region. Finally, in the tetralepton

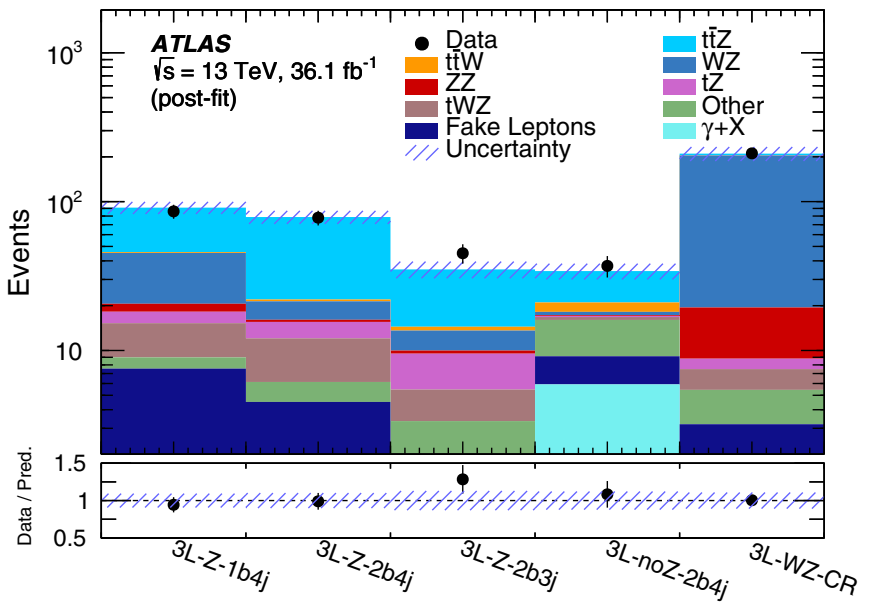

(a) channel, the four signal regions $4 \ell$-DF- $1 \mathrm{~b}, 4 \ell$-DF- $2 \mathrm{~b}, 4 \ell-$ SF- $1 \mathrm{~b}$ and $4 \ell$-SF- $2 \mathrm{~b}$ and the control region $4 \ell$-ZZ-CR are included in the fit.

The fit is based on the profile-likelihood technique, where systematic uncertainties are included in the fit as nuisance parameters constrained by Gaussian functions. None of the uncertainty parameters are found to be significantly constrained or pulled in the fit. The calculation of confidence intervals and hypothesis testing is performed using a modified frequentist method as implemented in RooStats [80,81].

Figure 10 shows the BDT output distribution in signal regions $2 \ell-\mathrm{Z}-6 \mathrm{j} 1 \mathrm{~b}, 2 \ell-\mathrm{Z}-5 \mathrm{j} 2 \mathrm{~b}$ and $2 \ell-\mathrm{Z}-6 \mathrm{j} 2 \mathrm{~b}$ after performing the fit. Figures 11 and 12 summarize the comparison between data and the post-fit signal and background yields for regions sensitive to $t \bar{t} Z$ and $t \bar{t} W$ production, together with the relevant control regions. In all cases, good agreement between observed values and the expectation is seen. The normalization corrections for the $W Z$ and $Z Z$ backgrounds with respect to the predictions are obtained from the fits as described in Sec. V and found to be compatible with unity: $0.91 \pm 0.10$ for the $W Z$ background and $1.11 \pm 0.17$ for the $Z Z$ background. The normalizations of the $Z+1 \mathrm{HF}$ and $Z+2 \mathrm{HF}$ backgrounds are mainly constrained in the low BDT output bins of the OS dilepton channel signal regions, where the signal contamination is low. Their values are found to be $1.19 \pm$ 0.25 and $1.09 \pm 0.13$, respectively.

In addition to the combined fit described above, fits in individual channels are performed. The $t \bar{t} Z$ signal strength is extracted through fits to the opposite-sign dilepton regions alone, to the trilepton channel regions alone and to the tetralepton channel signal regions alone. The $t \bar{t} W$ signal strength is extracted using the four trilepton signal

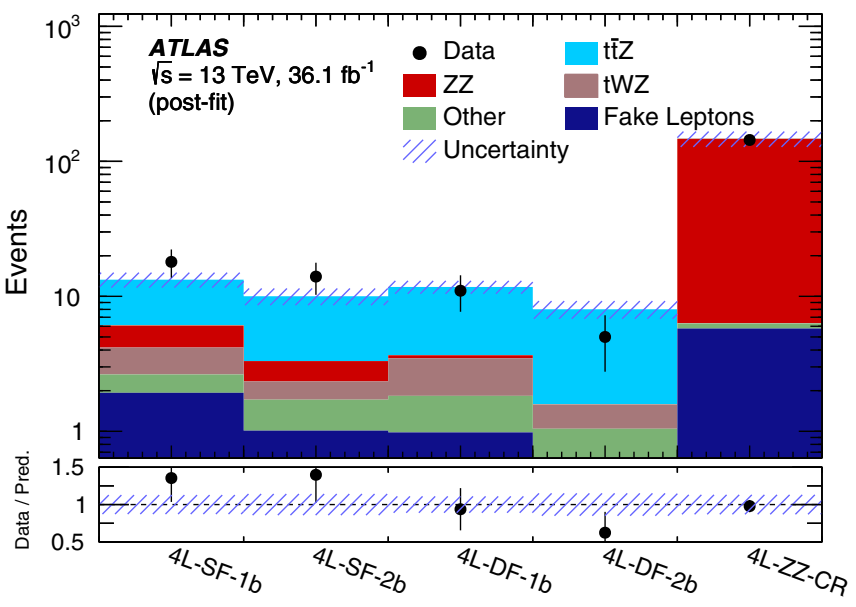

(b)

FIG. 11. Event yields in data compared with the results of the fit that extracts $\sigma_{t \bar{t} Z}$ and $\sigma_{t \bar{t} W}$ simultaneously in the (a) trilepton and (b) tetralepton signal regions targeting the $t \bar{t} Z$ process. Yields for the control regions used to extract the normalization of the $W Z$ and $Z Z$ backgrounds are also shown. The "Other" background summarizes all small SM backgrounds described in Sec. III. The shaded band represents the total uncertainty. 


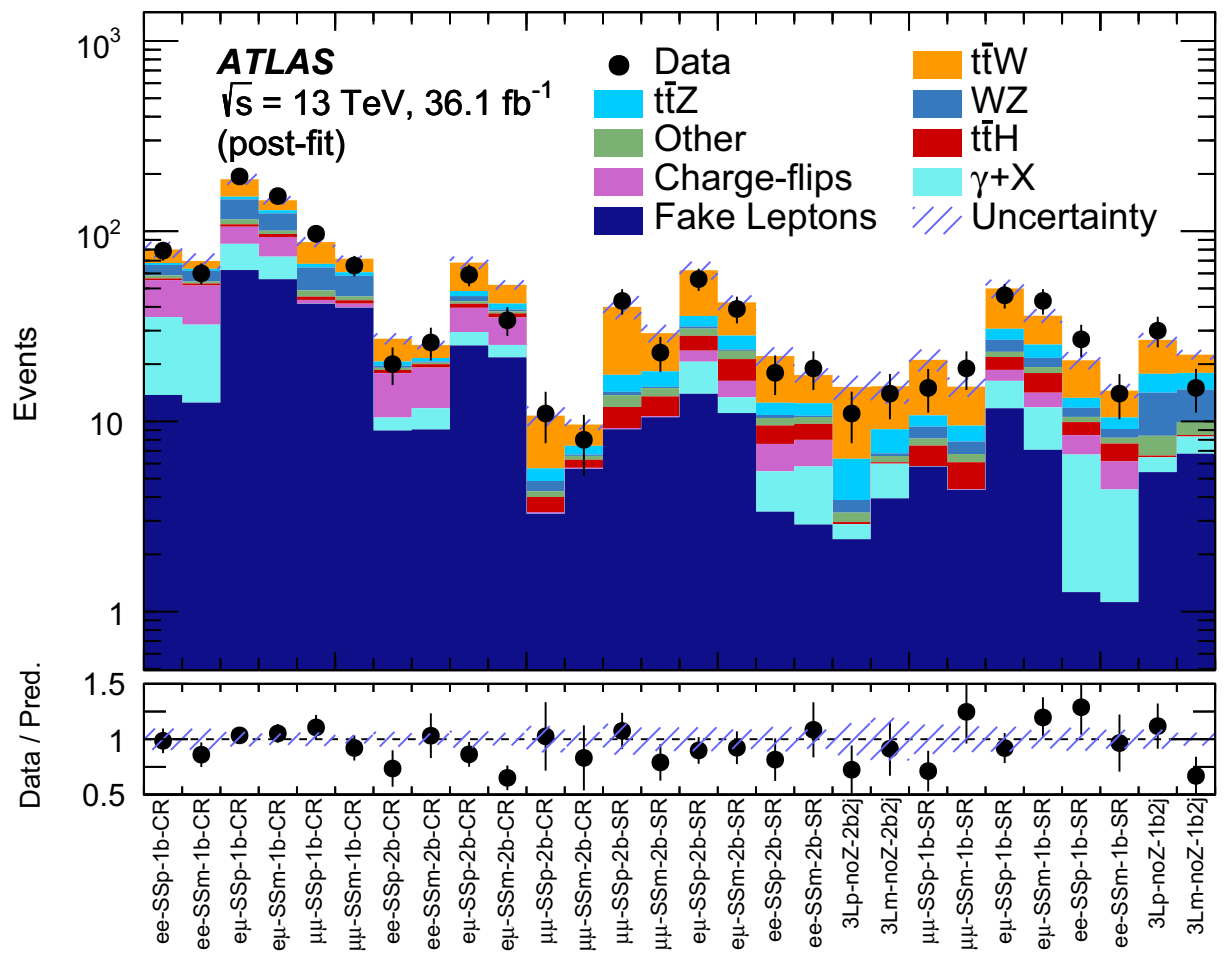

FIG. 12. Event yields in data compared with the results of the fit that extracts $\sigma_{\bar{t} \bar{t} Z}$ and $\sigma_{\bar{t} \bar{t} W}$ simultaneously in the regions targeting the $t \bar{t} W$ process. The "Other" background summarizes all small SM backgrounds described in Sec. III. The shaded band represents the total uncertainty.

regions targeting $t \bar{t} W$ and the same-sign dilepton regions considered in the combined fit. The measured values of the signal strengths $\mu_{t \bar{t} Z}$ and $\mu_{\bar{t} \bar{W}}$ are reported in Table VII for each channel separately and for the combined fit. Agreement is observed for the measured values between all the different fit configurations.

The measured signal strengths from the combined fit and their uncertainties are converted to inclusive cross-section measurements using the signal simulation described in Sec. III and the central values of the theoretical predictions. The results are: $\sigma_{t \bar{t} Z}=0.95 \pm 0.08_{\text {stat }} \pm 0.10_{\text {syst }} \mathrm{pb}=$ $0.95 \pm 0.13 \mathrm{pb}$ and $\sigma_{t \bar{t} W}=0.87 \pm 0.13_{\text {stat }} \pm 0.14_{\text {syst }} \mathrm{pb}=$ $0.87 \pm 0.19 \mathrm{pb}$. Figure 13 shows a comparison of the fit results with theoretical predictions, $\sigma_{t \bar{t} Z}^{\text {th }}=0.88_{-0.11}^{+0.09} \mathrm{pb}$ and $\sigma_{t \bar{t} W}^{\text {th }}=0.60_{-0.07}^{+0.08} \mathrm{pb}$, demonstrating good agreement between the measured and predicted cross sections.

TABLE VII. Measured signal strengths of $t \bar{t} Z$ and $t \bar{t} W$ for different fit configurations and the combined fit. The uncertainties include statistical and systematic components.

\begin{tabular}{lcc}
\hline \hline Fit configuration & $\mu_{t \bar{t} Z}$ & $\mu_{t \bar{t} W}$ \\
\hline Combined & $1.08 \pm 0.14$ & $1.44 \pm 0.32$ \\
$2 \ell$-OS & $0.73 \pm 0.28$ & $\cdots$ \\
$3 \ell t \bar{t} Z$ & $1.08 \pm 0.18$ & $\cdots$ \\
$2 \ell$-SS and $3 \ell t \bar{t} W$ & $\cdots$ & $1.41 \pm 0.33$ \\
$4 \ell$ & $1.21 \pm 0.29$ & $\cdots$ \\
\hline \hline
\end{tabular}

For the $t \bar{t} Z$ process, both the observed and the expected significances are found to be much larger than five standard deviations. For the $t \bar{t} W$ process, an excess of events over the expected background-only hypothesis is found with an

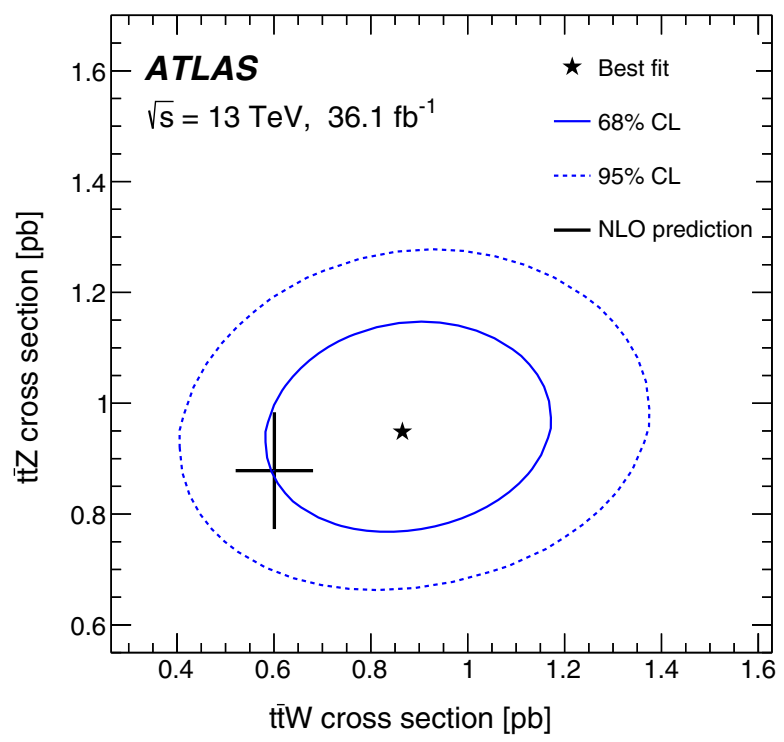

FIG. 13. The result of the simultaneous fit to the $t \bar{t} Z$ and $t \bar{t} W$ cross sections along with the $68 \%$ and $95 \%$ confidence level (C.L.) contours. The cross shows the SM calculations and their uncertainties, including renormalization and factorization scale uncertainties as well as uncertainties including $\alpha_{\mathrm{S}}$ variations. 
TABLE VIII. List of relative uncertainties in the measured cross sections of the $t \bar{t} Z$ and $t \bar{t} W$ processes from the fit, grouped in categories. All uncertainties are symmetrized. The sum in quadrature may not be equal to the total due to correlations between uncertainties introduced by the fit.

\begin{tabular}{lcc}
\hline \hline Uncertainty & $\sigma_{t \bar{t} Z}$ & $\sigma_{t \bar{t} W}$ \\
\hline Luminosity & $2.9 \%$ & $4.5 \%$ \\
Simulated sample statistics & $2.0 \%$ & $5.3 \%$ \\
Data-driven background statistics & $2.5 \%$ & $6.3 \%$ \\
JES/JER & $1.9 \%$ & $4.1 \%$ \\
Flavor tagging & $4.2 \%$ & $3.7 \%$ \\
Other object-related & $3.7 \%$ & $2.5 \%$ \\
Data-driven background normalization & $3.2 \%$ & $3.9 \%$ \\
Modeling of backgrounds from simulation & $5.3 \%$ & $2.6 \%$ \\
Background cross sections & $2.3 \%$ & $4.9 \%$ \\
Fake leptons and charge misID & $1.8 \%$ & $5.7 \%$ \\
$t \bar{t} Z$ modeling & $4.9 \%$ & $0.7 \%$ \\
$t \bar{t} W$ modeling & $0.3 \%$ & $8.5 \%$ \\
\hline Total systematic & $10 \%$ & $16 \%$ \\
Statistical & $8.4 \%$ & $15 \%$ \\
\hline Total & $13 \%$ & $22 \%$ \\
\hline \hline
\end{tabular}

observed (expected) significance of 4.3 (3.4) standard deviations. The significance values are computed using the asymptotic approximation described in Ref. [82].

Table VIII shows the uncertainties in the measured $t \bar{t} Z$ and $t \bar{t} W$ cross sections, grouped in categories, along with the total uncertainties. For both processes, the precision of the measurement is affected by statistical and systematic uncertainties in similar proportions. For the $t \bar{t} Z$ determination, the dominant systematic uncertainty sources are the modeling of the backgrounds and of the signal. For the $\bar{t} W$ determination, the dominant systematic uncertainty sources are the modeling of the signal and the limited amount of data available in the control regions and simulated event samples.

\section{INTERPRETATION}

The effective field theory (EFT) framework provides a model-independent approach to the parametrization of possible deviations from the SM predictions. In this framework, effects due to BSM physics are described by adding additional operators of dimension six or higher to the SM Lagrangian. Each EFT operator $\mathcal{O}_{i}$ is associated with a Wilson coefficient $C_{i}$, and the operators enter the modified Lagrangian in the form $\left(C_{i} / \Lambda^{2}\right) \mathcal{O}_{i}$, where $\Lambda$ is the characteristic energy scale of the BSM physics.

The complete set of independent, gauge-invariant and baryon-number conserving EFT operators at dimension six contains 59 different operators [83,84]. In the present analysis, five of these operators are considered, all of which modify the $t t Z$ vertex: $\mathcal{O}_{\phi Q}^{(3)}, \mathcal{O}_{\phi Q}^{(1)}, \mathcal{O}_{\phi t}, \mathcal{O}_{t W}, \mathcal{O}_{t B}$.
TABLE IX. Effective field theory operators considered and their form in terms of SM fields. The notation of Ref. [85] is used.

\begin{tabular}{lc}
\hline \hline Operator & Expression \\
\hline $\mathcal{O}_{\phi Q}^{(3)}$ & $\left(\phi^{\dagger} i \stackrel{\leftrightarrow}{D}{ }_{\mu}^{I} \phi\right)\left(\bar{Q} \gamma^{\mu} \tau^{I} Q\right)$ \\
$\mathcal{O}_{\phi Q}^{(1)}$ & $\left(\phi^{\dagger} i \stackrel{\leftrightarrow}{D} \phi\right)\left(\bar{Q} \gamma^{\mu} Q\right)$ \\
$\mathcal{O}_{\phi t}$ & $\left(\phi^{\dagger} i \stackrel{\leftrightarrow}{D} \phi\right)\left(\bar{t} \gamma^{\mu} t\right)$ \\
$\mathcal{O}_{t W}$ & $\left(\bar{Q} \sigma^{\mu \nu} \tau^{I} t\right) \tilde{\phi} W_{\mu \nu}^{I}$ \\
$\mathcal{O}_{t B}$ & $\left(\bar{Q} \sigma^{\mu \nu} t\right) \tilde{\phi} B_{\mu \nu}$ \\
\hline \hline
\end{tabular}

The operators are defined in Table IX, following Ref. [85]. The first two operators enter the $t t Z$ vertex as a linear combination, such that the measurement is sensitive to the difference $\mathcal{C}_{\phi Q}^{(3)}-\mathcal{C}_{\phi Q}^{(1)}$. For this paper, the effect of this combination is evaluated by varying $\mathcal{C}_{\phi Q}^{(3)}$ with $\mathcal{C}_{\phi Q}^{(1)}$ set to zero.

Considering only one EFT operator at a time, any observable, such as the $t \bar{t} Z$ event rate in a certain signal region, can be expressed as a quadratic function of the coefficient $C_{i}$ :

$\sigma_{\mathrm{tot}, i}=\sigma_{\mathrm{SM}}+\frac{\mathcal{C}_{i}}{(\Lambda / 1 \mathrm{TeV})^{2}} \sigma_{i}^{(1)}+\frac{\mathcal{C}_{i}^{2}}{(\Lambda / 1 \mathrm{TeV})^{4}} \sigma_{i i}^{(2)}$.

The term linear in $C_{i}$ on the right-hand side of Eq. (1) results from the interference of the BSM operators with the SM. For $\mathcal{C}_{i} / \Lambda^{2}$ of order $1 \mathrm{TeV}^{-2}$, the interference term dominates in Eq. (1) for $\mathcal{O}_{\phi Q}^{(3)}$ and $\mathcal{O}_{\phi t}$, while the quadratic term dominates for $\mathcal{O}_{t W}$ and $\mathcal{O}_{t B}$.

The values of $\sigma_{i}^{(1)}$ and $\sigma_{i i}^{(2)}$ are computed using simulated event samples generated with MG5_aMC interfaced to Pythia $8[11,86,87]$. The computation is performed at NLO, separately for all trilepton and tetralepton signal regions. The detector reconstruction efficiency is verified to be compatible between SM $t \bar{t} Z$ samples and samples with nonzero values of $C_{i}$, for ranges of $C_{i}$ considered here.

A fit is then performed to extract $\mathcal{C}_{i} / \Lambda^{2}$. The fit is similar to the one described in Sec. VII, except that only the four trilepton and four tetralepton signal regions targeting $t \bar{t} Z$ are used and a normalization uncertainty of $12 \%$, corresponding to the uncertainty in the NLO crosssection computation, is applied to the SM $t \bar{t} Z$ prediction. Uncertainties resulting from the limited sizes of $\mathrm{MC}$ samples used to derive the values of $\sigma_{i}^{(1)}$ and $\sigma_{i i}^{(2)}$ are propagated to the measured values of $\mathcal{C}_{i} / \Lambda^{2}$.

The profile-likelihood test statistic is defined as $-\Delta \log (L)=\log \left(\mathcal{L}\left(\hat{C}_{i}\right) / \mathcal{L}\left(C_{i}\right)\right)$, where $\mathcal{L}$ is the profile likelihood as a function of the Wilson coefficient $C_{i}$, and $\hat{C}_{i}$ is the best-fit value of $C_{i}$. Approximate confidence intervals for the Wilson coefficients are computed using the 
TABLE X. The expected and observed $68 \%$ and $95 \%$ confidence intervals, which include the value 0 , for $\mathcal{C}_{i} / \Lambda^{2}$ for the EFT coefficients $\mathcal{C}_{\phi Q}^{(3)}, \mathcal{C}_{\phi t}, \mathcal{C}_{t B}$ and $\mathcal{C}_{t W}$. The intervals for $\mathcal{C}_{\phi Q}^{(3)}$ are derived setting $\mathcal{C}_{\phi Q}^{(1)}$ to zero; the measurement is sensitive to the difference $\mathcal{C}_{\phi Q}^{(3)}-\mathcal{C}_{\phi Q}^{(1)}$. All results are obtained by varying one coefficient at the time and are given in units of $1 / \mathrm{TeV}^{2}$. Previous indirect $68 \%$ C.L. constraints [88] are also quoted. The previous direct constraints at $95 \%$ C.L. are obtained from Ref. [10] for $\mathcal{C}_{\phi t}$ and $\mathcal{C}_{t B}$ and from Refs. [89,90] for $\mathcal{C}_{\phi Q}^{(3)}$ and $\mathcal{C}_{t W}$, using Ref. [83] to translate the measurements into limits on the coefficients. Limits from fits for the EFT coefficients with only the linear term are also shown.

\begin{tabular}{lcccc}
\hline \hline Coefficients & $\mathcal{C}_{\phi Q}^{(3)} / \Lambda^{2}$ & $\mathcal{C}_{\phi t} / \Lambda^{2}$ & $\mathcal{C}_{t B} / \Lambda^{2}$ & $\mathcal{C}_{t W} / \Lambda^{2}$ \\
\hline Previous indirect constraints at 68\% C.L. & {$[-4.7,0.7]$} & {$[-0.1,3.7]$} & {$[-0.5,10]$} & {$[-1.6,0.8]$} \\
Previous direct constraints at 95\% C.L. & {$[-1.3,1.3]$} & {$[-9.7,8.3]$} & {$[-6.9,4.6]$} & {$[-0.2,0.7]$} \\
Expected limit at 68\% C.L. & {$[-2.1,1.9]$} & {$[-3.8,2.7]$} & {$[-2.9,3.0]$} & {$[-1.8,1.9]$} \\
Expected limit at 95\% C.L. & {$[-4.5,3.6]$} & {$[-23,4.9]$} & {$[-4.2,4.3]$} & {$[-2.6,2.6]$} \\
Observed limit at 68\% C.L. & {$[-1.0,2.7]$} & {$[-2.0,3.5]$} & {$[-3.7,3.5]$} & {$[-2.2,2.1]$} \\
Observed limit at 95\% C.L. & {$[-3.3,4.2]$} & {$[-25,5.5]$} & {$[-5.0,5.0]$} & {$[-2.9,2.9]$} \\
Expected limit at 68\% C.L. (linear) & {$[-1.9,2.0]$} & {$[-3.0,3.2]$} & $\ldots$ & $\ldots$ \\
Expected limit at 95\% C.L. (linear) & {$[-3.7,4.0]$} & {$[-5.8,6.3]$} & $\ldots$ & $\ldots$ \\
Observed limit at 68\% C.L. (linear) & {$[-1.0,2.9]$} & {$[-1.8,4.4]$} & $\ldots$ & $\ldots$ \\
Observed limit at 95\% C.L. (linear) & {$[-2.9,4.9]$} & {$[-4.8,7.5]$} & $\ldots$ & $\ldots$ \\
\hline \hline
\end{tabular}

formula $-\Delta \log (L)=\varepsilon$, where the threshold $\varepsilon$ is set to 0.5

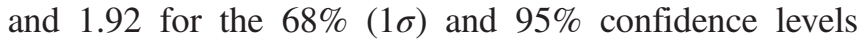
(C.L.), respectively.

The confidence intervals for $C_{i}$ are computed considering only the minimum of $\lambda\left(C_{i}\right)$ near $C_{i}=0$. For coefficient $\mathcal{C}_{\phi Q}^{(3)}\left(\mathcal{C}_{\phi t}\right)$, another, deeper minimum exists for negative values of $C_{i} \sim 30(20)$, which is excluded by previous constraints. The $68 \%$ and $95 \%$ confidence intervals are shown in Table X, together with previous constraints on the EFT coefficients obtained from Refs. [10,88-90]. The lower boundary of the $95 \%$ confidence interval for $\mathcal{C}_{\phi t}$ is at large negative values, which are excluded by indirect constraints. The $t \bar{t} Z$ measurement provides competitive constraints for positive $\mathcal{C}_{\phi t}$ values. The full likelihood scans are shown in Fig. 14 in the Appendix.

The fits are repeated while assuming that the quadratic terms are zero, and the results of these fits are also reported in Table X. For $\mathcal{C}_{t W}$ and $\mathcal{C}_{t B}$, where the quadratic terms dominate, the fits do not converge. Compared with the nominal fits for $\mathcal{C}_{\phi Q}^{(3)}$ and $\mathcal{C}_{\phi t}$, the limits shift to larger values, consistent with removing a positive term from the prediction. The most notable change is the improvement in the lower limit for $\mathcal{C}_{\phi t}$ at $95 \%$ C.L., as the second minimum disappears when a linear expression is assumed.

\section{CONCLUSION}

Measurements of the production cross sections of a topquark pair in association with a $Z$ or $W$ boson using $36.1 \mathrm{fb}^{-1}$ of data collected by the ATLAS detector in $\sqrt{s}=$ $13 \mathrm{TeV} p p$ collisions at the LHC are presented. Final states with two same- or opposite-sign leptons, three leptons or four leptons are analyzed. The $t \bar{t} Z$ and $t \bar{t} W$ production cross sections are determined to be $\sigma_{t \bar{t} Z}=0.95 \pm 0.08_{\text {stat }} \pm$ $0.10_{\text {syst }} \mathrm{pb}=0.95 \pm 0.13 \mathrm{pb}$ and $\sigma_{\bar{t} \bar{t} W}=0.87 \pm 0.13_{\text {stat }} \pm$ $0.14_{\text {syst }} \mathrm{pb}=0.87 \pm 0.19 \mathrm{pb}$. The measured values are consistent with the SM predictions. The measurements are used to derive confidence intervals for the Wilson coefficients of dimension- 6 effective field theory operators involving the top quark and the $Z$ boson.

\section{ACKNOWLEDGMENTS}

We thank CERN for the very successful operation of the LHC, as well as the support staff from our institutions without whom ATLAS could not be operated efficiently. We acknowledge the support of ANPCyT, Argentina; YerPhI, Armenia; ARC, Australia; BMWFW and FWF, Austria; ANAS, Azerbaijan; SSTC, Belarus; CNPq and FAPESP, Brazil; NSERC, NRC and CFI, Canada; CERN; CONICYT, Chile; CAS, MOST and NSFC, China; COLCIENCIAS, Colombia; MSMT CR, MPO CR and VSC CR, Czech Republic; DNRF and DNSRC, Denmark; IN2P3-CNRS, CEA-DRF/IRFU, France; SRNSFG, Georgia; BMBF, HGF, and MPG, Germany; GSRT, Greece; RGC, Hong Kong SAR, China; ISF and Benoziyo Center, Israel; INFN, Italy; MEXT and JSPS, Japan; CNRST, Morocco; NWO, Netherlands; RCN, Norway; MNiSW and NCN, Poland; FCT, Portugal; MNE/IFA, Romania; MES of Russia and NRC KI, Russian Federation; JINR; MESTD, Serbia; MSSR, Slovakia; ARRS and MIZŠ, Slovenia; DST/NRF, South Africa; MINECO, Spain; SRC and Wallenberg Foundation, Sweden; SERI, SNSF and Cantons of Bern and Geneva, Switzerland; MOST, Taiwan; TAEK, Turkey; STFC, 
United Kingdom; DOE and NSF, United States of America. In addition, individual groups and members have received support from BCKDF, CANARIE, CRC and Compute Canada, Canada; COST, ERC, ERDF, Horizon 2020, and Marie Skłodowska-Curie Actions, European Union; Investissements d' Avenir Labex and Idex, ANR, France; DFG and AvH Foundation, Germany; Herakleitos, Thales and Aristeia programmes co-financed by EU-ESF and the Greek NSRF, Greece; BSF-NSF and GIF, Israel; CERCA Programme Generalitat de Catalunya, Spain; The Royal
Society and Leverhulme Trust, United Kingdom. The crucial computing support from all WLCG partners is acknowledged gratefully, in particular from CERN, the ATLAS Tier-1 facilities at TRIUMF (Canada), NDGF (Denmark, Norway, Sweden), CC-IN2P3 (France), KIT/ GridKA (Germany), INFN-CNAF (Italy), NL-T1 (Netherlands), PIC (Spain), ASGC (Taiwan), RAL (UK) and BNL (USA), the Tier-2 facilities worldwide and large non-WLCG resource providers. Major contributors of computing resources are listed in Ref. [91].

\section{APPENDIX: ADDITIONAL MATERIAL}

The Appendix shows the profile-likelihood test statistic for the four EFT coefficients and the input variables used for the OS dilepton BDT.

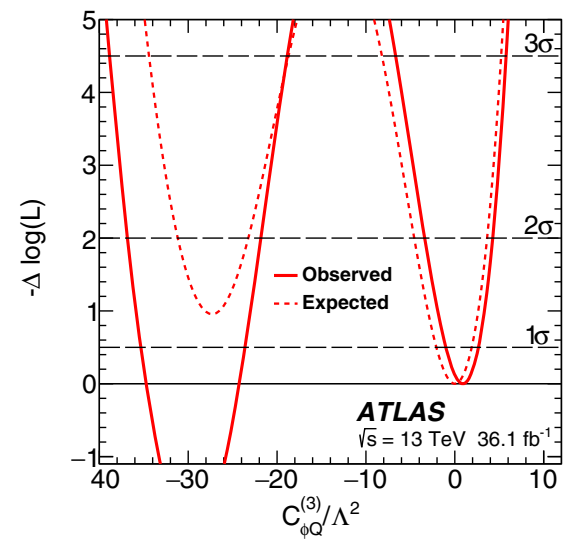

(a)

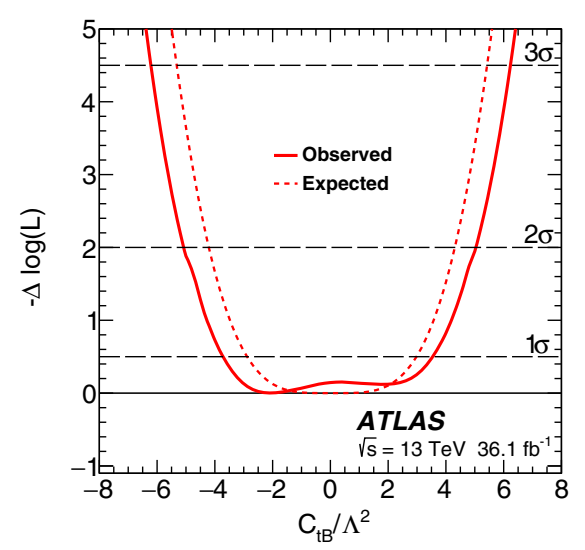

(c)

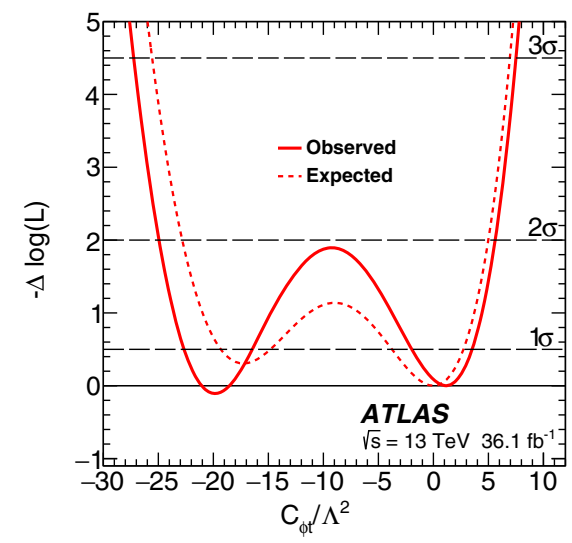

(b)

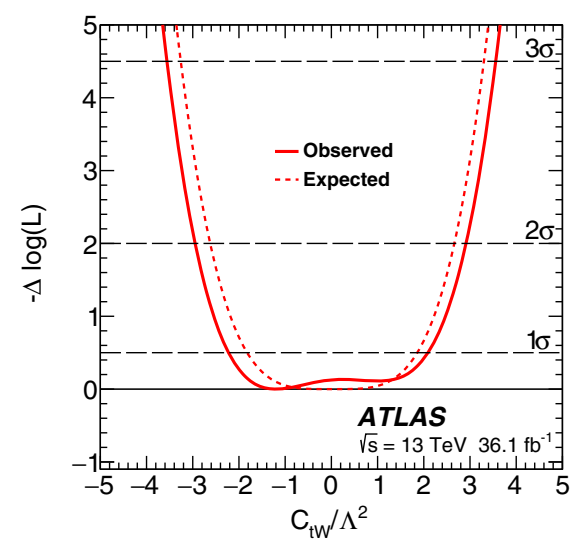

(d)

FIG. 14. The value of the profile-likelihood test statistic as a function of $c / \Lambda^{2}$, for (a) $\mathcal{C}_{\phi Q}^{(3)}$, (b) $\mathcal{C}_{\phi t}$, (c) $\mathcal{C}_{t B}$, and (d) $\mathcal{C}_{t W}$. In the $\mathcal{C}_{\phi Q}^{(3)}$ and $\mathcal{C}_{\phi t}$ distributions, another, deeper minimum exists for large negative values of $C_{i}$, which is excluded by indirect measurements. There, the vertical axis is chosen such that the value of the likelihood at the minimum near $C_{i}=0$ is zero. 
TABLE XI. The definitions and ranking of input variables for the BDT in the OS dilepton analysis. Jets and leptons are ordered in descending order of $p_{\mathrm{T}}$. Only the first eight jets are considered when calculating the input variables.

\begin{tabular}{|c|c|c|c|}
\hline \multirow[b]{2}{*}{ Definition } & \multicolumn{3}{|c|}{ Ranking } \\
\hline & $6 \mathrm{j} 1 \mathrm{~b}$ & $5 \mathrm{j} 2 \mathrm{~b}$ & $6 \mathrm{j} 2 \mathrm{~b}$ \\
\hline$p_{\mathrm{T}}$ of the lepton pair & 8 & 11 & 8 \\
\hline$p_{\mathrm{T}}$ of the fourth jet & 6 & 12 & 6 \\
\hline$p_{\mathrm{T}}$ of the fifth jet & .. & 14 & $\cdots$ \\
\hline$p_{\mathrm{T}}$ of the sixth jet & 9 & $\cdots$ & 11 \\
\hline$\Delta R_{\eta}$ between the two leptons & 7 & 8 & 12 \\
\hline Number of jet pairs with mass within a window of $30 \mathrm{GeV}$ around $85 \mathrm{GeV}$ & 4 & 6 & 4 \\
\hline $\begin{array}{l}\text { Number of three-jet combinations (containing exactly one } b \text {-tagged jet) compatible with a top quark } \\
\qquad\left(\left|m_{b j j}-m_{t}\right|<15 \mathrm{GeV}\right) \text { and }\left(\left|m_{j j}-m_{W}\right|<15 \mathrm{GeV}\right)\end{array}$ & .. & $\cdots$ & 17 \\
\hline Invariant mass of the two jets with the smallest $\Delta R_{\eta}$ & 13 & 7 & 14 \\
\hline Invariant mass of the two untagged jets with the highest $p_{\mathrm{T}}$ & 15 & 13 & .. \\
\hline Invariant mass of the two jets with the highest value of the $b$-tagging discriminant & $\cdots$ & 10 & 9 \\
\hline Scalar sum of $p_{\mathrm{T}}$ divided by the sum of energy of all jets & 2 & 1 & 2 \\
\hline Average $\Delta R_{\eta}$ of all jet pairs & 5 & 4 & 5 \\
\hline Maximum invariant mass of a lepton and the $b$-tagged jet with the smallest $\Delta R_{\eta}$ & 14 & $\cdots$ & 13 \\
\hline First Fox-Wolfram moment built from jets and leptons & 3 & 2 & 1 \\
\hline Sum of jet $p_{\mathrm{T}}$, using up to six jets & 12 & 5 & 10 \\
\hline$\eta$ of dilepton system & 1 & 3 & 3 \\
\hline Sum of the two closest two-jet invariant masses from $\mathrm{jjj}_{1}$ and $\mathrm{jjj}_{2}$ divided by two & 10 & $\cdots$ & 15 \\
\hline$\Delta R_{\eta}$ between two jets with the highest value of the $b$-tagging discriminant in the event & .. & 9 & 7 \\
\hline$p_{\mathrm{T}}$ of the $b$-tagged jet with the highest $p_{\mathrm{T}}$ & 11 & $\ldots$ & 16 \\
\hline
\end{tabular}

[1] ATLAS Collaboration, Evidence for the associated production of the Higgs boson and a top quark pair with the ATLAS detector, Phys. Rev. D 97, 072003 (2018).

[2] CMS Collaboration, Evidence for associated production of a Higgs boson with a top quark pair in final states with electrons, muons, and hadronically decaying $\tau$ leptons at $\sqrt{s}=13 \mathrm{TeV}$, J. High Energy Phys. 08 (2018) 066.

[3] CMS Collaboration, Observation of $t \bar{t} H$ Production, Phys. Rev. Lett. 120, 231801 (2018).

[4] ATLAS Collaboration, Observation of Higgs boson production in association with a top quark pair at the LHC with the ATLAS detector, Phys. Lett. B 784, 173 (2018).

[5] ATLAS Collaboration, Observation of top-quark pair production in association with a photon and measurement of the $t \bar{t} \gamma$ production cross section in $p p$ collisions at $\sqrt{s}=7 \mathrm{TeV}$ using the ATLAS detector, Phys. Rev. D 91, 072007 (2015).

[6] ATLAS Collaboration, Measurement of the $t \bar{t} \gamma$ production cross section in proton-proton collisions at $\sqrt{s}=8 \mathrm{TeV}$ with the ATLAS detector, J. High Energy Phys. 11 (2017) 086.

[7] CMS Collaboration, Measurement of the semileptonic $t \bar{t}+$ $\gamma$ production cross section in $p p$ collisions at $\sqrt{s}=8 \mathrm{TeV}$, J. High Energy Phys. 10 (2017) 006.
[8] ATLAS Collaboration, Measurements of inclusive and differential fiducial cross-sections of $t \bar{t} \gamma$ production in leptonic final states at $\sqrt{s}=13 \mathrm{TeV}$ in ATLAS, arXiv: 1812.01697.

[9] J. A. Dror, M. Farina, E. Salvioni, and J. Serra, Strong tW scattering at the LHC, J. High Energy Phys. 01 (2016) 071.

[10] A. Buckley, C. Englert, J. Ferrando, D. J. Miller, L. Moore, M. Russell, and C.D. White, Constraining top quark effective theory in the LHC Run II era, J. High Energy Phys. 04 (2016) 015.

[11] O. Bessidskaia Bylund, F. Maltoni, I. Tsinikos, E. Vryonidou, and C. Zhang, Probing top quark neutral couplings in the Standard Model effective field theory at NLO in QCD, J. High Energy Phys. 05 (2016) 052.

[12] S. Weinberg, Phenomenological Lagrangians, Physica 96A, 327 (1979).

[13] W. Buchmüller and D. Wyler, Effective Lagrangian analysis of new interactions and flavour conservation, Nucl. Phys. B268, 621 (1986).

[14] C. N. Leung, S. T. Love, and S. Rao, Low-energy manifestations of a new interactions scale: Operator analysis, Z. Phys. C 31, 433 (1986).

[15] ATLAS Collaboration, Measurement of the $t \bar{t} W$ and $t \bar{t} Z$ production cross sections in $p p$ collisions at $\sqrt{s}=8 \mathrm{TeV}$ with the ATLAS detector, J. High Energy Phys. 11 (2015) 172 . 
[16] ATLAS Collaboration, Measurement of the $t \bar{t} Z$ and $t \bar{t} W$ production cross sections in multilepton final states using $3.2 \mathrm{fb}^{-1}$ of $p p$ collisions at $\sqrt{s}=13 \mathrm{TeV}$ with the ATLAS detector, Eur. Phys. J. C 77, 40 (2017).

[17] CMS Collaboration, Observation of top quark pairs produced in association with a vector boson in $p p$ collisions at $\sqrt{s}=8 \mathrm{TeV}$, J. High Energy Phys. 01 (2016) 096.

[18] CMS Collaboration, Measurement of the cross section for top quark pair production in association with a $W$ or $Z$ boson in proton-proton collisions at $\sqrt{s}=13 \mathrm{TeV}$, J. High Energy Phys. 08 (2018) 011.

[19] ATLAS Collaboration, The ATLAS experiment at the CERN Large Hadron Collider, J. Instrum. 3, S08003 (2008).

[20] ATLAS Collaboration, Study of the mechanical stability of the ATLAS insertable B-layer, CERN Report No. ATLINDET-PUB-2015-001， 2015, https://cds.cern.ch/record/ 2022587.

[21] B. Abbott et al., Production and integration of the ATLAS insertable B-layer, J. Instrum. 13, T05008 (2018).

[22] ATLAS Collaboration, Performance of the ATLAS trigger system in 2015, Eur. Phys. J. C 77, 317 (2017).

[23] ATLAS Collaboration, Luminosity determination in $p p$ collisions at $\sqrt{s}=8 \mathrm{TeV}$ using the ATLAS detector at the LHC, Eur. Phys. J. C 76, 653 (2016).

[24] G. Avoni et al., The new LUCID-2 detector for luminosity measurement and monitoring in ATLAS, J. Instrum. 13, P07017 (2018).

[25] D. J. Lange, The EvtGen particle decay simulation package, Nucl. Instrum. Methods Phys. Res., Sect. A 462, 152 (2001).

[26] T. Gleisberg, S. Höche, F. Krauss, M. Schönherr, S. Schumann, F. Siegert, and J. Winter, Event generation with SHERPA 1.1, J. High Energy Phys. 02 (2009) 007.

[27] ATLAS Collaboration, The ATLAS simulation infrastructure, Eur. Phys. J. C 70, 823 (2010).

[28] S. Agostinelli et al., Geant4: A simulation toolkit, Nucl. Instrum. Methods Phys. Res., Sect. A 506, 250 (2003).

[29] ATLAS Collaboration, The simulation principle and performance of the ATLAS fast calorimeter simulation FastCaloSim, CERN Report No. ATL-PHYS-PUB-2010-013, 2010, https://cds.cern.ch/record/1300517.

[30] T. Sjöstrand, S. Ask, J. R. Christiansen, R. Corke, N. Desai, P. Ilten, S. Mrenna, S. Prestel, C. O. Rasmussen, and P. Z. Skands, An introduction to PYTHIA 8.2, Comput. Phys. Commun. 191, 159 (2015).

[31] ATLAS Collaboration, Further ATLAS tunes of Pythia 6 and Pythia 8, CERN Report No. ATL-PHYS-PUB-2011014, 2011, https://cds.cern.ch/record/1400677.

[32] J. Alwall, R. Frederix, S. Frixione, V. Hirschi, F. Maltoni, O. Mattelaer, H.-S. Shao, T. Stelzer, P. Torrielli, and M. Zaro, The automated computation of tree-level and next-toleading order differential cross sections, and their matching to parton shower simulations, J. High Energy Phys. 07 (2014) 079.

[33] S. Frixione, V. Hirschi, D. Pagani, H.-S. Shao, and M. Zaro, Electroweak and QCD corrections to top-pair hadroproduction in association with heavy bosons, J. High Energy Phys. 06 (2015) 184.

[34] D. de Florian et al., Handbook of LHC Higgs cross sections: 4. Deciphering the nature of the Higgs sector, arXiv: 1610.07922, DOI: 10.23731/CYRM-2017-002.

[35] R. D. Ball et al., Parton distributions for the LHC run II, J. High Energy Phys. 04 (2015) 040.

[36] ATLAS Collaboration, ATLAS Pythia 8 tunes to $7 \mathrm{TeV}$ data, CERN Report No. ATL-PHYS-PUB-2014-021, 2014, https://cds.cern.ch/record/1966419.

[37] R. D. Ball et al., Parton distributions with LHC data, Nucl. Phys. B867, 244 (2013).

[38] T. Sjöstrand, S. Mrenna, and P.Z. Skands, PYTHIA 6.4 physics and manual, J. High Energy Phys. 05 (2006) 026.

[39] J. Pumplin, D. R. Stump, J. Huston, H.-L. Lai, P. Nadolsky, and W.-K. Tung, New generation of parton distributions with uncertainties from global QCD analysis, J. High Energy Phys. 07 (2002) 012.

[40] P. Z. Skands, Tuning Monte Carlo generators: The Perugia tunes, Phys. Rev. D 82, 074018 (2010).

[41] T. Gleisberg and S. Höche, Comix, a new matrix element generator, J. High Energy Phys. 12 (2008) 039.

[42] F. Cascioli, P. Maierhöfer, and S. Pozzorini, Scattering Amplitudes with Open Loops, Phys. Rev. Lett. 108, 111601 (2012).

[43] S. Schumann and F. Krauss, A parton shower algorithm based on Catani-Seymour dipole factorisation, J. High Energy Phys. 03 (2008) 038.

[44] S. Höche, F. Krauss, M. Schönherr, and F. Siegert, QCD matrix elements + parton showers. The NLO case, J. High Energy Phys. 04 (2013) 027.

[45] R. Gavin, Y. Li, F. Petriello, and S. Quackenbush, FEWZ 2.0: A code for hadronic $\mathrm{Z}$ production at next-tonext-to-leading order, Comput. Phys. Commun. 182, 2388 (2011).

[46] H.-L. Lai, M. Guzzi, J. Huston, Z. Li, P. M. Nadolsky, J. Pumplin, and C.-P. Yuan, New parton distributions for collider physics, Phys. Rev. D 82, 074024 (2010).

[47] S. Frixione, G. Ridolfi, and P. Nason, A positive-weight next-to-leading-order Monte Carlo for heavy flavour hadroproduction, J. High Energy Phys. 09 (2007) 126.

[48] P. Nason, A new method for combining NLO QCD with shower Monte Carlo algorithms, J. High Energy Phys. 11 (2004) 040.

[49] S. Frixione, P. Nason, and C. Oleari, Matching NLO QCD computations with parton shower simulations: the POWHEG method, J. High Energy Phys. 11 (2007) 070.

[50] S. Alioli, P. Nason, C. Oleari, and E. Re, A general framework for implementing NLO calculations in shower Monte Carlo programs: The POWHEG BOX, J. High Energy Phys. 06 (2010) 043.

[51] M. Czakon and A. Mitov, Top++: A program for the calculation of the top-pair cross-section at hadron colliders, Comput. Phys. Commun. 185, 2930 (2014).

[52] E. Re, Single-top Wt-channel production matched with parton showers using the POWHEG method, Eur. Phys. J. C 71, 1547 (2011). 
[53] M. Aliev, H. Lacker, U. Langenfeld, S. Moch, P. Uwer, and M. Wiedermann, HATHOR: Hadronic top and heavy quarks cross section calculator, Comput. Phys. Commun. 182, 1034 (2011).

[54] P. Kant, O. M. Kind, T. Kintscher, T. Lohse, T. Martini, S. Mölbitz, P. Rieck, and P. Uwer, HatHor for single top-quark production: Updated predictions and uncertainty estimates for single top-quark production in hadronic collisions, Comput. Phys. Commun. 191, 74 (2015).

[55] N. Kidonakis, Top quark production, arXiv:1311.0283.

[56] V. Barger, W.-Y. Keung, and B. Yencho, Triple-top signal of new physics at the LHC, Phys. Lett. B 687, 70 (2010).

[57] G. Bevilacqua and M. Worek, Constraining BSM physics at the LHC: Four top final states with NLO accuracy in perturbative QCD, J. High Energy Phys. 07 (2012) 111.

[58] ATLAS Collaboration, Electron efficiency measurements with the ATLAS detector using 2012 LHC proton-proton collision data, Eur. Phys. J. C 77, 195 (2017).

[59] ATLAS Collaboration, Muon reconstruction performance of the ATLAS detector in proton-proton collision data at $\sqrt{s}=13$ TeV, Eur. Phys. J. C 76, 292 (2016).

[60] M. Cacciari, G. P. Salam, and G. Soyez, The anti- $k_{t}$ jet clustering algorithm, J. High Energy Phys. 04 (2008) 063.

[61] M. Cacciari and G. P. Salam, Dispelling the $N^{3}$ myth for the $k_{t}$ jet-finder, Phys. Lett. B 641, 57 (2006).

[62] ATLAS Collaboration, Topological cell clustering in the ATLAS calorimeters and its performance in LHC Run 1, Eur. Phys. J. C 77, 490 (2017).

[63] M. Cacciari, G. P. Salam, and G. Soyez, The catchment area of jets, J. High Energy Phys. 04 (2008) 005.

[64] ATLAS Collaboration, Jet energy scale measurements and their systematic uncertainties in proton-proton collisions at $\sqrt{s}=13 \mathrm{TeV}$ with the ATLAS detector, Phys. Rev. D 96, 072002 (2017).

[65] ATLAS Collaboration, Performance of pile-up mitigation techniques for jets in $p p$ collisions at $\sqrt{s}=8 \mathrm{TeV}$ using the ATLAS detector, Eur. Phys. J. C 76, 581 (2016).

[66] ATLAS Collaboration, Measurements of $b$-jet tagging efficiency with the ATLAS detector using $t \bar{t}$ events at $\sqrt{s}=13 \mathrm{TeV}$, J. High Energy Phys. 08 (2018) 089.

[67] ATLAS Collaboration, Performance of algorithms that reconstruct missing transverse momentum in $\sqrt{s}=8 \mathrm{TeV}$ proton-proton collisions in the ATLAS detector, Eur. Phys. J. C 77, 241 (2017).

[68] ATLAS Collaboration, Performance of missing transverse momentum reconstruction with the ATLAS detector using proton-proton collisions at $\sqrt{s}=13 \mathrm{TeV}$, Eur. Phys. J. C 78, 903 (2018).

[69] ATLAS Collaboration, Measurement of the top quark-pair production cross section with ATLAS in $p p$ collisions at $\sqrt{s}=7$ TeV, Eur. Phys. J. C 71, 1577 (2011).

[70] G. C. Fox and S. Wolfram, Observables for the Analysis of Event Shapes in $e^{+} e^{-}$Annihilation and Other Processes, Phys. Rev. Lett. 41, 1581 (1978).
[71] ATLAS Collaboration, Electron reconstruction and identification efficiency measurements with the ATLAS detector using the 2011 LHC proton-proton collision data, Eur. Phys. J. C 74, 2941 (2014).

[72] ATLAS Collaboration, Electron efficiency measurements with the ATLAS detector using the 2012 LHC protonproton collision data, CERN Report No. ATLAS-CONF2014-032, 2014, https://cds.cern.ch/record/1706245.

[73] ATLAS Collaboration, Electron identification measurements in ATLAS using $\sqrt{s}=13 \mathrm{TeV}$ data with $50 \mathrm{~ns}$ bunch spacing, CERN Report No. ATL-PHYS-PUB-2015041, 2015, https://cds.cern.ch/record/2048202.

[74] ATLAS Collaboration, Measurement of $b$-tagging efficiency of $c$-jets in $t \bar{t}$ events using a likelihood approach with the ATLAS detector, CERN Report No. ATLASCONF-2018-001, 2018, https://cds.cern.ch/record/2306649.

[75] ATLAS Collaboration, Calibration of light-flavour $b$-jet mistagging rates using ATLAS proton-proton collision data at $\sqrt{s}=13 \mathrm{TeV}$, CERN Report No. ATLAS-CONF-2018006, 2018, https://cds.cern.ch/record/2314418.

[76] ATLAS Collaboration, Calibration of $b$-tagging using dileptonic top pair events in a combinatorial likelihood approach with the ATLAS experiment, CERN Report No. ATLASCONF-2014-004, 2014, https://cds.cern.ch/record/1664335.

[77] ATLAS Collaboration, Modelling of the $t \bar{t} H$ and $t \bar{t} V(V=$ $W, Z$ ) processes for $\sqrt{s}=13 \mathrm{TeV}$ ATLAS analyses, CERN Report No. ATL-PHYS-PUB-2016-005, 2016, https://cds .cern.ch/record/2120826.

[78] ATLAS Collaboration, Measurement of the production cross-section of a single top quark in association with a $Z$ boson in proton-proton collisions at $13 \mathrm{TeV}$ with the ATLAS detector, Phys. Lett. B 780, 557 (2018).

[79] CMS Collaboration, Measurement of the associated production of a single top quark and a $Z$ boson in $p p$ collisions at $\sqrt{s}=13 \mathrm{TeV}$, Phys. Lett. B 779, 358 (2018).

[80] W. Verkerke and D. Kirkby, The RooFit toolkit for data modeling, arXiv:physics/0306116.

[81] W. Verkerke and D. Kirkby, RooFit users manual v2.91, http://roofit.sourceforge.net.

[82] G. Cowan, K. Cranmer, E. Gross, and O. Vitells, Asymptotic formulae for likelihood-based tests of new physics, Eur. Phys. J. C 71, 1554 (2011).

[83] J. A. Aguilar-Saavedra, A minimal set of top anomalous couplings, Nucl. Phys. B812, 181 (2009).

[84] B. Grzadkowski, M. Iskrzynski, M. Misiak, and J. Rosiek, Dimension-six terms in the Standard Model Lagrangian, J. High Energy Phys. 10 (2010) 085.

[85] J. A. Aguilar-Saavedra et al., Interpreting top-quark LHC measurements in the Standard-Model effective field theory, arXiv: 1802.07237.

[86] N. D. Christensen and C. Duhr, FeynRules: Feynman rules made easy, Comput. Phys. Commun. 180, 1614 (2009).

[87] C. Degrande, C. Duhr, B. Fuks, D. Grellscheid, O. Mattelaer, and T. Reiter, UFO: The Universal FeynRules Output, Comput. Phys. Commun. 183, 1201 (2012).

[88] C. Zhang, N. Greiner, and S. Willenbrock, Constraints on nonstandard top quark couplings, Phys. Rev. D 86, 014024 (2012). 
[89] CMS Collaboration, Measurement of the $t$-channel singletop-quark production cross section and of the $\left|V_{t b}\right| \mathrm{CKM}$ matrix element in $p p$ collisions at $\sqrt{s}=8 \mathrm{TeV}$, J. High Energy Phys. 06 (2014) 090.

[90] ATLAS Collaboration, Measurement of the $W$ boson polarisation in $t \bar{t}$ events from $p p$ collisions at $\sqrt{s}=8 \mathrm{TeV}$ in the lepton + jets channel with ATLAS, Eur. Phys. J. C 77, 264 (2017).

[91] ATLAS Collaboration, ATLAS computing acknowledgements, CERN Report No. ATL-GEN-PUB-2016-002, https://cds.cern.ch/record/2202407.

M. Aaboud ${ }^{34 \mathrm{~d}}$ G. Aad, ${ }^{99}$ B. Abbott, ${ }^{125}$ D. C. Abbott, ${ }^{100}$ O. Abdinov, ${ }^{13, a}$ B. Abeloos, ${ }^{129}$ D. K. Abhayasinghe, ${ }^{91}$ S. H. Abidi, ${ }^{164}$ O. S. AbouZeid,${ }^{39}$ N. L. Abraham, ${ }^{153}$ H. Abramowicz, ${ }^{158}$ H. Abreu, ${ }^{157}$ Y. Abulaiti, ${ }^{6}$ B. S. Acharya, ${ }^{64 a, 64 b, b}$ S. Adachi, ${ }^{160}$ L. Adam, ${ }^{97}$ L. Adamczyk, ${ }^{81 a}$ L. Adamek, ${ }^{164}$ J. Adelman, ${ }^{119}$ M. Adersberger, ${ }^{112}$ A. Adiguzel, ${ }^{12 c, c}$ T. Adye, ${ }^{141}$ A. A. Affolder, ${ }^{143}$ Y. Afik, ${ }^{157}$ C. Agheorghiesei, ${ }^{27 \mathrm{c}}$ J. A. Aguilar-Saavedra, ${ }^{137 f, 137 \mathrm{a}, \mathrm{d}}$ F. Ahmadov, ${ }^{77, \mathrm{e}}$ G. Aielli, ${ }^{71 \mathrm{a}, 71 \mathrm{~b}}$ S. Akatsuka, ${ }^{83}$ T. P. A. Åkesson, ${ }^{94}$ E. Akilli, ${ }^{52}$ A. V. Akimov ${ }^{108}$ G. L. Alberghi, ${ }^{23 b, 23 a}$ J. Albert,${ }^{173}$ P. Albicocco, ${ }^{49}$ M. J. Alconada Verzini, ${ }^{86}$ S. Alderweireldt, ${ }^{117}$ M. Aleksa, ${ }^{35}$ I. N. Aleksandrov, ${ }^{77}$ C. Alexa, ${ }^{27 b}$ D. Alexandre, ${ }^{19}$ T. Alexopoulos, ${ }^{10}$ M. Alhroob, ${ }^{125}$ B. Ali, ${ }^{139}$ G. Alimonti, ${ }^{66 a}$ J. Alison, ${ }^{36}$ S. P. Alkire, ${ }^{145}$ C. Allaire, ${ }^{129}$ B. M. M. Allbrooke, ${ }^{153}$ B. W. Allen, ${ }^{128}$ P. P. Allport, ${ }^{21}$ A. Aloisio, ${ }^{67 a, 67 b}$ A. Alonso, ${ }^{39}$ F. Alonso, ${ }^{86}$ C. Alpigiani, ${ }^{145}$ A. A. Alshehri,${ }^{55}$ M. I. Alstaty ${ }^{99}$ B. Alvarez Gonzalez, ${ }^{35}$ D. Álvarez Piqueras, ${ }^{171}$ M. G. Alviggi,${ }^{67 a, 67 b}$ B. T. Amadio, ${ }^{18}$ Y. Amaral Coutinho, ${ }^{78 b}$ A. Ambler, ${ }^{101}$ L. Ambroz, ${ }^{132}$ C. Amelung, ${ }^{26}$ D. Amidei, ${ }^{103}$ S. P. Amor Dos Santos, ${ }^{137 a, 137 c}$ S. Amoroso, ${ }^{44}$ C. S. Amrouche,${ }^{52}$ F. An, ${ }^{76}$ C. Anastopoulos ${ }^{146}$ L. S. Ancu, ${ }^{52}$ N. Andari, ${ }^{142}$ T. Andeen, ${ }^{11}$ C. F. Anders,${ }^{59 b}$ J. K. Anders,${ }^{20}$ K. J. Anderson ${ }^{36}$ A. Andreazza, ${ }^{66 a, 66 b}$ V. Andrei, ${ }^{59 a}$ C. R. Anelli, ${ }^{173}$ S. Angelidakis, ${ }^{37}$ I. Angelozzi, ${ }^{118}$ A. Angerami, ${ }^{38}$ A. V. Anisenkov, ${ }^{120 b, 120 a}$ A. Annovi, ${ }^{69 a}$ C. Antel, ${ }^{59 a}$ M. T. Anthony, ${ }^{146}$ M. Antonelli, ${ }^{49}$ D. J. A. Antrim, ${ }^{168}$ F. Anulli, ${ }^{70 a}$ M. Aoki, ${ }^{79}$ J. A. Aparisi Pozo, ${ }^{171}$ L. Aperio Bella, ${ }^{35}$ G. Arabidze, ${ }^{104}$ J. P. Araque, ${ }^{137 a}$ V. Araujo Ferraz,${ }^{78 b}$ R. Araujo Pereira, ${ }^{78 b}$ A. T. H. Arce, ${ }^{47}$ R. E. Ardell, ${ }^{91}$ F. A. Arduh,${ }^{86}$ J-F. Arguin, ${ }^{107}$ S. Argyropoulos, ${ }^{75}$ J.-H. Arling, ${ }^{44}$ A. J. Armbruster, ${ }^{35}$ L. J. Armitage, ${ }^{90}$ A. Armstrong, ${ }^{168}$ O. Arnaez, ${ }^{164}$ H. Arnold, ${ }^{118}$ M. Arratia, ${ }^{31}$ O. Arslan, ${ }^{24}$ A. Artamonov, ${ }^{109, a}$ G. Artoni, ${ }^{132}$ S. Artz ${ }^{97}$ S. Asai ${ }^{160}$ N. Asbah,${ }^{57}$ E. M. Asimakopoulou, ${ }^{169}$ L. Asquith ${ }^{153}$ K. Assamagan, ${ }^{29}$ R. Astalos,${ }^{28 a}$ R. J. Atkin, ${ }^{32 a}$ M. Atkinson, ${ }^{170}$ N. B. Atlay, ${ }^{148}$ K. Augsten, ${ }^{139}$ G. Avolio, ${ }^{35}$ R. Avramidou, ${ }^{58 a}$ M. K. Ayoub, ${ }^{15 a}$ A. M. Azoulay, ${ }^{165 b}$

G. Azuelos, ${ }^{107, f}$ A. E. Baas, ${ }^{59 \mathrm{a}}$ M. J. Baca, ${ }^{21}$ H. Bachacou, ${ }^{142}$ K. Bachas,${ }^{65 a, 65 b}$ M. Backes,${ }^{132}$ P. Bagnaia, ${ }^{70 a, 70 b}$ M. Bahmani, ${ }^{82}$ H. Bahrasemani, ${ }^{149}$ A. J. Bailey,${ }^{171}$ V. R. Bailey, ${ }^{170}$ J. T. Baines,${ }^{141}$ M. Bajic, ${ }^{39}$ C. Bakalis ${ }^{10}$ O. K. Baker, ${ }^{180}$ P. J. Bakker, ${ }^{118}$ D. Bakshi Gupta, ${ }^{8}$ S. Balaji ${ }^{154}$ E. M. Baldin, ${ }^{120 b, 120 a}$ P. Balek, ${ }^{177}$ F. Balli, ${ }^{142}$ W. K. Balunas, ${ }^{134}$ J. Balz, ${ }^{97}$ E. Banas, ${ }^{82}$ A. Bandyopadhyay, ${ }^{24}$ S. Banerjee, ${ }^{178, g}$ A. A. E. Bannoura, ${ }^{179}$ L. Barak ${ }^{158}$ W. M. Barbe,${ }^{37}$ E. L. Barberio, ${ }^{102}$ D. Barberis, ${ }^{53 b, 53 a}$ M. Barbero, ${ }^{99}$ T. Barillari, ${ }^{113}$ M-S. Barisits, ${ }^{35}$ J. Barkeloo, ${ }^{128}$ T. Barklow, ${ }^{150}$ R. Barnea, ${ }^{157}$ S. L. Barnes,${ }^{58 c}$ B. M. Barnett, ${ }^{141}$ R. M. Barnett, ${ }^{18}$ Z. Barnovska-Blenessy, ${ }^{58 a}$ A. Baroncelli, ${ }^{72 a}$ G. Barone, ${ }^{29}$ A. J. Barr, ${ }^{132}$

L. Barranco Navarro, ${ }^{171}$ F. Barreiro, ${ }^{96}$ J. Barreiro Guimarães da Costa, ${ }^{15 a}$ R. Bartoldus,${ }^{150}$ A. E. Barton, ${ }^{87}$ P. Bartos, ${ }^{28 a}$ A. Basalaev, ${ }^{135}$ A. Bassalat, ${ }^{129}$ R. L. Bates, ${ }^{55}$ S. J. Batista, ${ }^{164}$ S. Batlamous, ${ }^{34 \mathrm{e}}$ J. R. Batley, ${ }^{31}$ M. Battaglia, ${ }^{143}$ M. Bauce, ${ }^{70 a}, 70 \mathrm{~b}$ F. Bauer, ${ }^{142}$ K. T. Bauer, ${ }^{168}$ H. S. Bawa, ${ }^{150}$ J. B. Beacham, ${ }^{123}$ T. Beau, ${ }^{133}$ P. H. Beauchemin, ${ }^{167}$ P. Bechtle, ${ }^{24}$ H. C. Beck, ${ }^{51}$ H. P. Beck,${ }^{20, \mathrm{~h}}$ K. Becker, ${ }^{50}$ M. Becker ${ }^{97}$ C. Becot, ${ }^{44}$ A. Beddall, ${ }^{12 \mathrm{~d}}$ A. J. Beddall, ${ }^{12 \mathrm{a}}$ V. A. Bednyakov, ${ }^{77}$ M. Bedognetti, ${ }^{118}$ C. P. Bee, ${ }^{152}$ T. A. Beermann, ${ }^{74}$ M. Begalli, ${ }^{78 b}$ M. Begel,${ }^{29}$ A. Behera, ${ }^{152}$ J. K. Behr, ${ }^{44}$ F. Beisiegel, ${ }^{24}$ A. S. Bell,${ }^{92}$ G. Bella, ${ }^{158}$ L. Bellagamba, ${ }^{23 b}$ A. Bellerive, ${ }^{33}$ M. Bellomo, ${ }^{157}$ P. Bellos,${ }^{9}$ K. Belotskiy, ${ }^{110}$ N. L. Belyaev, ${ }^{110}$ O. Benary, ${ }^{158, a}$ D. Benchekroun, ${ }^{34 a}$ M. Bender, ${ }^{112}$ N. Benekos, ${ }^{10}$ Y. Benhammou, ${ }^{158}$ E. Benhar Noccioli, ${ }^{180}$ J. Benitez, ${ }^{75}$ D. P. Benjamin, ${ }^{6}$ M. Benoit,${ }^{52}$ J. R. Bensinger, ${ }^{26}$ S. Bentvelsen, ${ }^{118}$ L. Beresford, ${ }^{132}$ M. Beretta, ${ }^{49}$ D. Berge,${ }^{44}$ E. Bergeaas Kuutmann, ${ }^{169}$ N. Berger, ${ }^{5}$ B. Bergmann, ${ }^{139}$ L. J. Bergsten, ${ }^{26}$ J. Beringer, ${ }^{18}$ S. Berlendis, ${ }^{7}$ N. R. Bernard, ${ }^{100}$ G. Bernardi, ${ }^{133}$ C. Bernius, ${ }^{150}$ F. U. Bernlochner ${ }^{24}$ T. Berry, ${ }^{91}$ P. Berta,${ }^{97}$ C. Bertella, ${ }^{15 a}$ G. Bertoli, ${ }^{43 a, 43 b}$ I. A. Bertram ${ }^{87}$ G. J. Besjes ${ }^{39}$

O. Bessidskaia Bylund, ${ }^{179}$ M. Bessner, ${ }^{44}$ N. Besson, ${ }^{142}$ A. Bethani, ${ }^{98}$ S. Bethke, ${ }^{113}$ A. Betti, ${ }^{24}$ A. J. Bevan, ${ }^{90}$ J. Beyer, ${ }^{113}$ R. Bi, ${ }^{136}$ R. M. Bianchi, ${ }^{136}$ O. Biebel, ${ }^{112}$ D. Biedermann, ${ }^{19}$ R. Bielski, ${ }^{35}$ K. Bierwagen, ${ }^{97}$ N. V. Biesuz,${ }^{69 a, 69 b}$ M. Biglietti, ${ }^{72 a}$ T. R. V. Billoud, ${ }^{107}$ M. Bindi, ${ }^{51}$ A. Bingul, ${ }^{12 \mathrm{~d}}$ C. Bini, ${ }^{70 a, 70 \mathrm{~b}}$ S. Biondi, ${ }^{23 \mathrm{~b}, 23 \mathrm{a}}$ M. Birman, ${ }^{177}$ T. Bisanz, ${ }^{51}$ J. P. Biswal, ${ }^{158}$ C. Bittrich, ${ }^{46}$ D. M. Bjergaard, ${ }^{47}$ J. E. Black, ${ }^{150}$ K. M. Black,${ }^{25}$ T. Blazek, ${ }^{28 a}$ I. Bloch, ${ }^{44}$ C. Blocker, ${ }^{26}$ A. Blue, ${ }^{55}$ U. Blumenschein, ${ }^{90}$ Dr. Blunier, ${ }^{144 a}$ G. J. Bobbink, ${ }^{118}$ V. S. Bobrovnikov, ${ }^{120 b, 120 a}$ S. S. Bocchetta, ${ }^{94}$ A. Bocci, ${ }^{47}$ D. Boerner, ${ }^{179}$ D. Bogavac, ${ }^{112}$ A. G. Bogdanchikov, ${ }^{120 b, 120 a}$ C. Bohm, ${ }^{43 a}$ V. Boisvert, ${ }^{91}$ P. Bokan,${ }^{51,169}$ T. Bold, ${ }^{81 a}$ A. S. Boldyrev, ${ }^{111}$ A. E. Bolz,${ }^{59 b}$ M. Bomben, ${ }^{133}$ M. Bona,${ }^{90}$ J. S. Bonilla, ${ }^{128}$ M. Boonekamp, ${ }^{142}$ H. M. Borecka-Bielska, ${ }^{88}$ 
A. Borisov, ${ }^{121}$ G. Borissov, ${ }^{87}$ J. Bortfeldt, ${ }^{35}$ D. Bortoletto, ${ }^{132}$ V. Bortolotto, ${ }^{71,71 b}$ D. Boscherini,${ }^{23 b}$ M. Bosman, ${ }^{14}$ J. D. Bossio Sola, ${ }^{30}$ K. Bouaouda, ${ }^{34 a}$ J. Boudreau, ${ }^{136}$ E. V. Bouhova-Thacker, ${ }^{87}$ D. Boumediene, ${ }^{37}$ C. Bourdarios, ${ }^{129}$ S. K. Boutle,${ }^{55}$ A. Boveia, ${ }^{123}$ J. Boyd, ${ }^{35}$ D. Boye,${ }^{32 b, i}$ I. R. Boyko, ${ }^{77}$ A. J. Bozson, ${ }^{91}$ J. Bracinik, ${ }^{21}$ N. Brahimi, ${ }^{99}$ A. Brandt, ${ }^{8}$ G. Brandt, ${ }^{179}$ O. Brandt, ${ }^{59 a}$ F. Braren, ${ }^{44}$ U. Bratzler ${ }^{161}$ B. Brau, ${ }^{100}$ J. E. Brau, ${ }^{128}$ W. D. Breaden Madden,${ }^{55}$ K. Brendlinger, ${ }^{44}$ L. Brenner, ${ }^{44}$ R. Brenner, ${ }^{169}$ S. Bressler, ${ }^{177}$ B. Brickwedde, ${ }^{97}$ D. L. Briglin, ${ }^{21}$ D. Britton, ${ }^{55}$ D. Britzger, ${ }^{113}$ I. Brock, ${ }^{24}$ R. Brock, ${ }^{104}$ G. Brooijmans, ${ }^{38}$ T. Brooks, ${ }^{91}$ W. K. Brooks, ${ }^{144 b}$ E. Brost,${ }^{119}$ J. H Broughton, ${ }^{21}$ P. A. Bruckman de Renstrom,${ }^{82}$ D. Bruncko, ${ }^{28 b}$ A. Bruni, ${ }^{23 b}$ G. Bruni, ${ }^{23 b}$ L. S. Bruni, ${ }^{118}$ S. Bruno, ${ }^{71 a, 71 b}$ B. H. Brunt, ${ }^{31}$ M. Bruschi, ${ }^{23 b}$ N. Bruscino, ${ }^{136}$ P. Bryant ${ }^{36}$ L. Bryngemark, ${ }^{94}$ T. Buanes, ${ }^{17}$ Q. Buat,${ }^{35}$ P. Buchholz, ${ }^{148}$ A. G. Buckley, ${ }^{55}$ I. A. Budagov, ${ }^{77}$ M. K. Bugge, ${ }^{131}$ F. Bührer, ${ }^{50}$ O. Bulekov, ${ }^{110}$ D. Bullock, ${ }^{8}$ T. J. Burch, ${ }^{119}$ S. Burdin, ${ }^{88}$ C. D. Burgard,${ }^{118}$ A. M. Burger, ${ }^{5}$ B. Burghgrave,${ }^{8}$ K. Burka, ${ }^{82}$ S. Burke, ${ }^{141}$ I. Burmeister, ${ }^{45}$ J. T. P. Burr, ${ }^{132}$ V. Büscher, ${ }^{97}$ E. Buschmann, ${ }^{51}$ P. Bussey,${ }^{55}$ J. M. Butler, ${ }^{25}$ C. M. Buttar, ${ }^{55}$ J. M. Butterworth, ${ }^{92}$ P. Butti ${ }^{35}$ W. Buttinger, ${ }^{35}$ A. Buzatu, ${ }^{155}$ A. R. Buzykaev, ${ }^{120 b, 120 a}$ G. Cabras, ${ }^{23 b, 23 a}$ S. Cabrera Urbán, ${ }^{171}$ D. Caforio, ${ }^{139}$ H. Cai, ${ }^{170}$ V. M. M. Cairo, ${ }^{2}$ O. Cakir, ${ }^{4 a}$ N. Calace, ${ }^{35}$ P. Calafiura, ${ }^{18}$ A. Calandri, ${ }^{99}$ G. Calderini, ${ }^{133}$ P. Calfayan, ${ }^{63}$ G. Callea, ${ }^{55}$ L. P. Caloba, ${ }^{78 b}$ S. Calvente Lopez, ${ }^{96}$ D. Calvet, ${ }^{37}$ S. Calvet, ${ }^{37}$ T. P. Calvet, ${ }^{152}$ M. Calvetti, ${ }^{69 a, 69 b}$ R. Camacho Toro, ${ }^{133}$ S. Camarda, ${ }^{35}$ D. Camarero Munoz, ${ }^{96}$ P. Camarri, ${ }^{71 a, 71 b}$ D. Cameron, ${ }^{131}$ R. Caminal Armadans, ${ }^{100}$ C. Camincher, ${ }^{35}$ S. Campana, ${ }^{35}$ M. Campanelli, ${ }^{92}$ A. Camplani, ${ }^{39}$ A. Campoverde,${ }^{148}$ V. Canale, ${ }^{67 a, 67 b}$ M. Cano Bret, ${ }^{58 \mathrm{c}}$ J. Cantero, ${ }^{126}$ T. Cao, ${ }^{158}$ Y. Cao, ${ }^{170}$ M. D. M. Capeans Garrido, ${ }^{35}$ I. Caprini, ${ }^{27 b}$ M. Caprini, ${ }^{27 b}$ M. Capua, ${ }^{40 b, 40 a}$ R. M. Carbone, ${ }^{38}$ R. Cardarelli, ${ }^{71 a}$ F. C. Cardillo, ${ }^{146}$ I. Carli, ${ }^{140}$ T. Carli, ${ }^{35}$ G. Carlino, ${ }^{67 a}$ B. T. Carlson, ${ }^{136}$ L. Carminati, ${ }^{66 a, 66 b}$ R. M. D. Carney, ${ }^{43 a, 43 b}$ S. Caron, ${ }^{117}$ E. Carquin, ${ }^{144 b}$ S. Carrá, ${ }^{66 a, 66 b}$ J. W. S. Carter, ${ }^{164}$ D. Casadei, ${ }^{32 b}$ M. P. Casado, ${ }^{14, j}$ A. F. Casha, ${ }^{164}$ D. W. Casper, ${ }^{168}$ R. Castelijn,${ }^{118}$ F. L. Castillo, ${ }^{171}$ V. Castillo Gimenez, ${ }^{171}$ N. F. Castro, ${ }^{137 a, 137 \mathrm{e}}$ A. Catinaccio, ${ }^{35}$ J. R. Catmore, ${ }^{131}$ A. Cattai,${ }^{35}$ J. Caudron, ${ }^{24}$ V. Cavaliere,${ }^{29}$ E. Cavallaro, ${ }^{14}$ D. Cavalli, ${ }^{66 a}$ M. Cavalli-Sforza, ${ }^{14}$ V. Cavasinni, ${ }^{69 a, 69 b}$ E. Celebi, ${ }^{12 b}$ F. Ceradini, ${ }^{72 a, 72 b}$ L. Cerda Alberich, ${ }^{171}$ A. S. Cerqueira, ${ }^{78 a}$ A. Cerri, ${ }^{153}$ L. Cerrito, ${ }^{71 a, 71 b}$ F. Cerutti, ${ }^{18}$ A. Cervelli, ${ }^{23 b, 23 a}$ S. A. Cetin, ${ }^{12 b}$ A. Chafaq, ${ }^{34 a}$ D. Chakraborty, ${ }^{119}$ S. K. Chan, ${ }^{57}$ W. S. Chan, ${ }^{118}$ W. Y. Chan, ${ }^{88}$ J. D. Chapman, ${ }^{31}$ B. Chargeishvili, ${ }^{156 b}$ D. G. Charlton, ${ }^{21}$ C. C. Chau, ${ }^{33}$ C. A. Chavez Barajas, ${ }^{153}$ S. Che, ${ }^{123}$ A. Chegwidden, ${ }^{104}$ S. Chekanov, ${ }^{6}$ S. V. Chekulaev, ${ }^{165 a}$ G. A. Chelkov, ${ }^{77, k}$ M. A. Chelstowska, ${ }^{35}$ B. Chen,${ }^{76}$ C. Chen, ${ }^{58 a}$ C. H. Chen, ${ }^{76}$ H. Chen, ${ }^{29}$ J. Chen, ${ }^{58 \mathrm{a}}$ J. Chen, ${ }^{38}$ S. Chen, ${ }^{134}$ S. J. Chen, ${ }^{15 \mathrm{c}}$ X. Chen, ${ }^{15 \mathrm{~b}, 1}$ Y. Chen, ${ }^{80}$ Y-H. Chen, ${ }^{44}$ H. C. Cheng, ${ }^{61 \mathrm{a}}$ H. J. Cheng, ${ }^{15 \mathrm{~d}}$ A. Cheplakov, ${ }^{77}$ E. Cheremushkina, ${ }^{121}$ R. Cherkaoui El Moursli, ${ }^{34 \mathrm{e}}$ E. Cheu, ${ }^{7}$ K. Cheung, ${ }^{62}$ T. J. A. Chevalérias, ${ }^{142}$ L. Chevalier, ${ }^{142}$ V. Chiarella, ${ }^{49}$ G. Chiarelli, ${ }^{69 a}$ G. Chiodini, ${ }^{65 a}$ A. S. Chisholm, ${ }^{35,21}$ A. Chitan, ${ }^{27 b}$ I. Chiu, ${ }^{160}$ Y. H. Chiu, ${ }^{173}$ M. V. Chizhov, ${ }^{77}$ K. Choi, ${ }^{63}$ A. R. Chomont, ${ }^{129}$ S. Chouridou, ${ }^{159}$ Y. S. Chow, ${ }^{118}$

V. Christodoulou, ${ }^{92}$ M. C. Chu, ${ }^{61 a}$ J. Chudoba, ${ }^{138}$ A. J. Chuinard, ${ }^{101}$ J. J. Chwastowski, ${ }^{82}$ L. Chytka, ${ }^{127}$ D. Cinca, ${ }^{45}$ V. Cindro, ${ }^{89}$ I. A. Cioară, ${ }^{24}$ A. Ciocio, ${ }^{18}$ F. Cirotto, ${ }^{67 a, 67 b}$ Z. H. Citron, ${ }^{177}$ M. Citterio, ${ }^{66 a}$ A. Clark, ${ }^{52}$ M. R. Clark, ${ }^{38}$ P. J. Clark, ${ }^{48}$ C. Clement, ${ }^{43 a, 43 b}$ Y. Coadou, ${ }^{99}$ M. Cobal, ${ }^{64 a, 64 c}$ A. Coccaro, ${ }^{53 b}$ J. Cochran,${ }^{76}$ H. Cohen, ${ }^{158}$ A. E. C. Coimbra, ${ }^{177}$ L. Colasurdo, ${ }^{117}$ B. Cole, ${ }^{38}$ A. P. Colijn, ${ }^{118}$ J. Collot, ${ }^{56}$ P. Conde Muiño, ${ }^{137 a, m}$ E. Coniavitis, ${ }^{50}$ S. H. Connell, ${ }^{32 b}$

I. A. Connelly, ${ }^{98}$ S. Constantinescu, ${ }^{27 b}$ F. Conventi, ${ }^{67 a, n}$ A. M. Cooper-Sarkar, ${ }^{132}$ F. Cormier, ${ }^{172}$ K. J. R. Cormier, ${ }^{164}$ L. D. Corpe ${ }^{92}$ M. Corradi, ${ }^{70 a, 70 b}$ E. E. Corrigan, ${ }^{94}$ F. Corriveau, ${ }^{101, o}$ A. Cortes-Gonzalez, ${ }^{35}$ M. J. Costa, ${ }^{171}$ F. Costanza, ${ }^{5}$ D. Costanzo, ${ }^{146}$ G. Cottin, ${ }^{31}$ G. Cowan, ${ }^{91}$ J. W. Cowley, ${ }^{31}$ B. E. Cox, ${ }^{98}$ J. Crane,${ }^{98}$ K. Cranmer,${ }^{122}$ S. J. Crawley, ${ }^{55}$ R. A. Creager, ${ }^{134}$ G. Cree, ${ }^{33}$ S. Crépé-Renaudin, ${ }^{56}$ F. Crescioli, ${ }^{133}$ M. Cristinziani, ${ }^{24}$ V. Croft, ${ }^{122}$ G. Crosetti, ${ }^{40 b, 40 a}$ A. Cueto, ${ }^{96}$ T. Cuhadar Donszelmann, ${ }^{146}$ A. R. Cukierman, ${ }^{150}$ S. Czekierda, ${ }^{82}$ P. Czodrowski, ${ }^{35}$

M. J. Da Cunha Sargedas De Sousa, ${ }^{58 b}$ C. Da Via,${ }^{98}$ W. Dabrowski, ${ }^{81 a}$ T. Dado, ${ }^{28 a, p}$ S. Dahbi, ${ }^{34 \mathrm{e}}$ T. Dai, ${ }^{103}$ F. Dallaire, ${ }^{107}$ C. Dallapiccola, ${ }^{100}$ M. Dam, ${ }^{39}$ G. D'amen, ${ }^{23 b, 23 a}$ J. Damp, ${ }^{97}$ J. R. Dandoy, ${ }^{134}$ M. F. Daneri, ${ }^{30}$ N. P. Dang, ${ }^{178, g}$ N. D Dann, ${ }^{98}$ M. Danninger, ${ }^{172}$ V. Dao, ${ }^{35}$ G. Darbo, ${ }^{53 \mathrm{~b}}$ S. Darmora, ${ }^{8}$ O. Dartsi, ${ }^{5}$ A. Dattagupta, ${ }^{128}$ T. Daubney, ${ }^{44}$ S. D' Auria, ${ }^{66 a, 66 \mathrm{~b}}$

W. Davey, ${ }^{24}$ C. David, ${ }^{44}$ T. Davidek, ${ }^{140}$ D. R. Davis, ${ }^{47}$ E. Dawe, ${ }^{102}$ I. Dawson, ${ }^{146}$ K. De,${ }^{8}$ R. De Asmundis,${ }^{67 a}$ A. De Benedetti, ${ }^{125}$ M. De Beurs, ${ }^{118}$ S. De Castro, ${ }^{23 b, 23 a}$ S. De Cecco, ${ }^{70 a, 70 b}$ N. De Groot, ${ }^{117}$ P. de Jong, ${ }^{118}$ H. De la Torre, ${ }^{104}$ F. De Lorenzi, ${ }^{76}$ A. De Maria,${ }^{69,69 b}$ D. De Pedis, ${ }^{70 a}$ A. De Salvo, ${ }^{70 a}$ U. De Sanctis, ${ }^{71 a, 71 b}$ M. De Santis ${ }^{71 a, 71 b}$ A. De Santo, ${ }^{153}$

K. De Vasconcelos Corga, ${ }^{99}$ J. B. De Vivie De Regie, ${ }^{129}$ C. Debenedetti, ${ }^{143}$ D. V. Dedovich, ${ }^{77}$ N. Dehghanian, ${ }^{3}$ M. Del Gaudio, ${ }^{40 \mathrm{~b}, 40 \mathrm{a}}$ J. Del Peso, ${ }^{96}$ Y. Delabat Diaz,${ }^{44}$ D. Delgove, ${ }^{129}$ F. Deliot,${ }^{142}$ C. M. Delitzsch, ${ }^{7}$ M. Della Pietra, ${ }^{67 \mathrm{a}, 67 \mathrm{~b}}$ D. Della Volpe, ${ }^{52}$ A. Dell'Acqua ${ }^{35}$ L. Dell'Asta, ${ }^{25}$ M. Delmastro, ${ }^{5}$ C. Delporte, ${ }^{129}$ P. A. Delsart, ${ }^{56}$ D. A. DeMarco, ${ }^{164}$ S. Demers ${ }^{180}$ M. Demichev, ${ }^{77}$ S. P. Denisov, ${ }^{121}$ D. Denysiuk, ${ }^{118}$ L. D’Eramo, ${ }^{133}$ D. Derendarz,${ }^{82}$ J. E. Derkaoui, ${ }^{34 \mathrm{~d}}$ F. Derue, ${ }^{133}$ P. Dervan,${ }^{88}$ K. Desch,${ }^{24}$ C. Deterre,${ }^{44}$ K. Dette, ${ }^{164}$ M. R. Devesa ${ }^{30}$ P. O. Deviveiros,${ }^{35}$ A. Dewhurst ${ }^{141}$ S. Dhaliwal, ${ }^{26}$ F. A. Di Bello, ${ }^{52}$ A. Di Ciaccio, ${ }^{71 a, 71 b}$ L. Di Ciaccio, ${ }^{5}$ W. K. Di Clemente, ${ }^{134}$ C. Di Donato, ${ }^{67 a, 67 b}$ 
A. Di Girolamo, ${ }^{35}$ G. Di Gregorio, ${ }^{69 a, 69 b}$ B. Di Micco,${ }^{72 a, 72 b}$ R. Di Nardo, ${ }^{100}$ K. F. Di Petrillo, ${ }^{57}$ R. Di Sipio, ${ }^{164}$ D. Di Valentino, ${ }^{33}$ C. Diaconu, ${ }^{99}$ M. Diamond, ${ }^{164}$ F. A. Dias, ${ }^{39}$ T. Dias Do Vale, ${ }^{137 a}$ M. A. Diaz, ${ }^{144 a}$ J. Dickinson, ${ }^{18}$ E. B. Diehl, ${ }^{103}$ J. Dietrich, ${ }^{19}$ S. Díez Cornell, ${ }^{44}$ A. Dimitrievska, ${ }^{18}$ J. Dingfelder, ${ }^{24}$ F. Dittus, ${ }^{35}$ F. Djama, ${ }^{99}$ T. Djobava, ${ }^{156 b}$ J. I. Djuvsland, ${ }^{17}$ M. A. B. Do Vale, ${ }^{78 c}$ M. Dobre, ${ }^{27 b}$ D. Dodsworth, ${ }^{26}$ C. Doglioni, ${ }^{94}$ J. Dolejsi, ${ }^{140}$ Z. Dolezal, ${ }^{140}$ M. Donadelli, ${ }^{78 d}$ J. Donini, ${ }^{37}$ A. D'onofrio, ${ }^{90}$ M. D'Onofrio ${ }^{88}$ J. Dopke, ${ }^{141}$ A. Doria, ${ }^{67 a}$ M. T. Dova ${ }^{86}$ A. T. Doyle ${ }^{55}$ E. Drechsler, ${ }^{149}$ E. Dreyer, ${ }^{149}$ T. Dreyer, ${ }^{51}$ Y. Du, ${ }^{58 b}$ F. Dubinin, ${ }^{108}$ M. Dubovsky, ${ }^{28 a}$ A. Dubreuil,${ }^{52}$ E. Duchovni, ${ }^{177}$ G. Duckeck, ${ }^{112}$ A. Ducourthial, ${ }^{133}$ O. A. Ducu, ${ }^{107, q}$ D. Duda, ${ }^{113}$ A. Dudarev, ${ }^{35}$ A. C. Dudder, ${ }^{97}$ E. M. Duffield, ${ }^{18}$ L. Duflot, ${ }^{129}$ M. Dührssen, ${ }^{35}$ C. Dülsen, ${ }^{179}$ M. Dumancic, ${ }^{177}$ A. E. Dumitriu, ${ }^{27 b, r}$ A. K. Duncan, ${ }^{55}$ M. Dunford, ${ }^{59 a}$ A. Duperrin, ${ }^{99}$ H. Duran Yildiz, ${ }^{4 a}$ M. Düren,${ }^{54}$ A. Durglishvili, ${ }^{156 b}$ D. Duschinger ${ }^{46}$ B. Dutta, ${ }^{44}$ D. Duvnjak, ${ }^{1}$ G. Dyckes, ${ }^{134}$ M. Dyndal, ${ }^{44}$ S. Dysch,${ }^{98}$ B. S. Dziedzic, ${ }^{82}$ K. M. Ecker, ${ }^{113}$ R. C. Edgar, ${ }^{103}$ T. Eifert, ${ }^{35}$ G. Eigen, ${ }^{17}$ K. Einsweiler, ${ }^{18}$ T. Ekelof, ${ }^{169}$ M. El Kacimi, ${ }^{34 \mathrm{c}}$ R. El Kosseifi, ${ }^{99}$ V. Ellajosyula, ${ }^{99}$ M. Ellert, ${ }^{169}$ F. Ellinghaus, ${ }^{179}$ A. A. Elliot, ${ }^{90}$ N. Ellis, ${ }^{35}$ J. Elmsheuser, ${ }^{29}$ M. Elsing, ${ }^{35}$ D. Emeliyanov, ${ }^{141}$ A. Emerman, ${ }^{38}$ Y. Enari, ${ }^{160}$ J. S. Ennis, ${ }^{175}$ M. B. Epland, ${ }^{47}$ J. Erdmann, ${ }^{45}$ A. Ereditato, ${ }^{20}$ S. Errede, ${ }^{170}$ M. Escalier, ${ }^{129}$ C. Escobar, ${ }^{171}$ O. Estrada Pastor, ${ }^{171}$ A. I. Etienvre, ${ }^{142}$ E. Etzion, ${ }^{158}$ H. Evans,${ }^{63}$ A. Ezhilov, ${ }^{135}$ M. Ezzi ${ }^{34 \mathrm{e}}$ F. Fabbri, ${ }^{55}$ L. Fabbri, ${ }^{23 b, 23 a}$ V. Fabiani, ${ }^{117}$ G. Facini, ${ }^{92}$ R. M. Faisca Rodrigues Pereira, ${ }^{137 a}$

R. M. Fakhrutdinov, ${ }^{121}$ S. Falciano, ${ }^{70 a}$ P. J. Falke, ${ }^{5}$ S. Falke, ${ }^{5}$ J. Faltova, ${ }^{140}$ Y. Fang, ${ }^{15 a}$ M. Fanti, ${ }^{66 a, 66 b}$ A. Farbin, ${ }^{8}$ A. Farilla, ${ }^{72 a}$ E. M. Farina, ${ }^{68 a, 68 b}$ T. Farooque, ${ }^{104}$ S. Farrell, ${ }^{18}$ S. M. Farrington, ${ }^{175}$ P. Farthouat, ${ }^{35}$ F. Fassi, ${ }^{34 \mathrm{e}}$ P. Fassnacht,${ }^{35}$ D. Fassouliotis, ${ }^{9}$ M. Faucci Giannelli, ${ }^{48}$ W. J. Fawcett, ${ }^{31}$ L. Fayard, ${ }^{129}$ O. L. Fedin, ${ }^{135, \mathrm{~s}}$ W. Fedorko, ${ }^{172}$ M. Feickert, ${ }^{41}$ S. Feigl, ${ }^{131}$ L. Feligioni, ${ }^{99}$ C. Feng, ${ }^{58 b}$ E. J. Feng, ${ }^{35}$ M. Feng, ${ }^{47}$ M. J. Fenton,${ }^{55}$ A. B. Fenyuk, ${ }^{121}$ J. Ferrando, ${ }^{44}$ A. Ferrari, ${ }^{169}$ P. Ferrari, ${ }^{118}$ R. Ferrari, ${ }^{68 \mathrm{a}}$ D. E. Ferreira de Lima ${ }^{59 b}$ A. Ferrer, ${ }^{171}$ D. Ferrere,${ }^{52}$ C. Ferretti, ${ }^{103}$ F. Fiedler, ${ }^{97}$ A. Filipčičc, ${ }^{89}$ F. Filthaut, ${ }^{117}$ K. D. Finelli, ${ }^{25}$ M. C. N. Fiolhais, ${ }^{137 a, 137 c, t}$ L. Fiorini, ${ }^{171}$ C. Fischer, ${ }^{14}$ W. C. Fisher,${ }^{104}$ N. Flaschel, ${ }^{44}$ I. Fleck,${ }^{148}$ P. Fleischmann, ${ }^{103}$ R. R. M. Fletcher, ${ }^{134}$ T. Flick, ${ }^{179}$ B. M. Flierl, ${ }^{112}$ L. M. Flores, ${ }^{134}$ L. R. Flores Castillo, ${ }^{61 a}$ F. M. Follega, ${ }^{73 a, 73 b}$ N. Fomin, ${ }^{17}$ G. T. Forcolin, ${ }^{73 a, 73 b}$ A. Formica, ${ }^{142}$ F. A. Förster ${ }^{14}$ A. C. Forti, ${ }^{98}$ A. G. Foster, ${ }^{21}$ D. Fournier, ${ }^{129}$ H. Fox, ${ }^{87}$ S. Fracchia, ${ }^{146}$ P. Francavilla, ${ }^{69,69 b}$ M. Franchini, ${ }^{23 b, 23 a}$ S. Franchino, ${ }^{59 a}$ D. Francis, ${ }^{35}$ L. Franconi,${ }^{143}$ M. Franklin, ${ }^{57}$ M. Frate ${ }^{168}$ A. N. Fray,${ }^{90}$ D. Freeborn, ${ }^{92}$ B. Freund, ${ }^{107}$ W. S. Freund, ${ }^{78 b}$ E. M. Freundlich, ${ }^{45}$ D. C. Frizzell, ${ }^{125}$ D. Froidevaux,${ }^{35}$ J. A. Frost, ${ }^{132}$ C. Fukunaga, ${ }^{161}$ E. Fullana Torregrosa, ${ }^{171}$ E. Fumagalli, ${ }^{53 b, 53 a}$ T. Fusayasu, ${ }^{114}$ J. Fuster, ${ }^{171}$ O. Gabizon, ${ }^{157}$ A. Gabrielli, ${ }^{23 b, 23 a}$ A. Gabrielli, ${ }^{18}$ G. P. Gach, ${ }^{81 a}$ S. Gadatsch, ${ }^{52}$ P. Gadow, ${ }^{113}$ G. Gagliardi, ${ }^{53 b, 53 a}$ L. G. Gagnon, ${ }^{107}$ C. Galea, ${ }^{27 b}$ B. Galhardo, ${ }^{137 a, 137 \mathrm{c}}$ E. J. Gallas, ${ }^{132}$ B. J. Gallop, ${ }^{141}$ P. Gallus, ${ }^{139}$

G. Galster, ${ }^{39}$ R. Gamboa Goni, ${ }^{90}$ K. K. Gan, ${ }^{123}$ S. Ganguly, ${ }^{177}$ J. Gao, ${ }^{58 a}$ Y. Gao,${ }^{88}$ Y. S. Gao, ${ }^{150, u}$ C. García, ${ }^{171}$ J. E. García Navarro, ${ }^{171}$ J. A. García Pascual, ${ }^{15 a}$ C. Garcia-Argos, ${ }^{50}$ M. Garcia-Sciveres, ${ }^{18}$ R. W. Gardner, ${ }^{36}$ N. Garelli, ${ }^{150}$ S. Gargiulo, ${ }^{50}$ V. Garonne, ${ }^{131}$ K. Gasnikova, ${ }^{44}$ A. Gaudiello, ${ }^{53 b 53 a}$ G. Gaudio, ${ }^{68 a}$ I. L. Gavrilenko, ${ }^{108}$ A. Gavrilyuk, ${ }^{109}$ C. Gay, ${ }^{172}$ G. Gaycken, ${ }^{24}$ E. N. Gazis, ${ }^{10}$ C. N. P. Gee, ${ }^{141}$ J. Geisen, ${ }^{51}$ M. Geisen, ${ }^{97}$ M. P. Geisler, ${ }^{59 a}$ C. Gemme,${ }^{53 b}$ M. H. Genest ${ }^{56}$ C. Geng, ${ }^{103}$ S. Gentile, ${ }^{70 a, 70 b}$ S. George, ${ }^{91}$ D. Gerbaudo, ${ }^{14}$ G. Gessner, ${ }^{45}$ S. Ghasemi, ${ }^{148}$

M. Ghasemi Bostanabad, ${ }^{173}$ M. Ghneimat, ${ }^{24}$ B. Giacobbe, ${ }^{23 b}$ S. Giagu, ${ }^{70 a}, 70 b$ N. Giangiacomi, ${ }^{23 b, 23 a}$ P. Giannetti, ${ }^{69 a}$ A. Giannini, ${ }^{67 a, 67 b}$ S. M. Gibson, ${ }^{91}$ M. Gignac, ${ }^{143}$ D. Gillberg, ${ }^{33}$ G. Gilles, ${ }^{179}$ D. M. Gingrich, ${ }^{3, f}$ M. P. Giordani, ${ }^{64 a, 64 c}$ F. M. Giorgi, ${ }^{23 b}$ P. F. Giraud, ${ }^{142}$ P. Giromini,${ }^{57}$ G. Giugliarelli, ${ }^{64 a, 64 c}$ D. Giugni, ${ }^{66 a}$ F. Giuli, ${ }^{132}$ M. Giulini, ${ }^{59 b}$ S. Gkaitatzis, ${ }^{159}$ I. Gkialas, ${ }^{9, v}$ E. L. Gkougkousis, ${ }^{14}$ P. Gkountoumis,${ }^{10}$ L. K. Gladilin, ${ }^{111}$ C. Glasman, ${ }^{96}$ J. Glatzer, ${ }^{14}$ P. C. F. Glaysher,${ }^{44}$ A. Glazov, ${ }^{44}$ M. Goblirsch-Kolb, ${ }^{26}$ J. Godlewski, ${ }^{82}$ S. Goldfarb, ${ }^{102}$ T. Golling, ${ }^{52}$ D. Golubkov, ${ }^{121}$ A. Gomes, ${ }^{137 a, 137 b}$ R. Goncalves Gama, ${ }^{51}$ R. Gonçalo, ${ }^{137 a}$ G. Gonella, ${ }^{50}$ L. Gonella, ${ }^{21}$ A. Gongadze, ${ }^{77}$ F. Gonnella, ${ }^{21}$ J. L. Gonski, ${ }^{57}$ S. González de la Hoz, ${ }^{171}$ S. Gonzalez-Sevilla, ${ }^{52}$ L. Goossens, ${ }^{35}$ P. A. Gorbounov, ${ }^{109}$ H. A. Gordon, ${ }^{29}$ B. Gorini ${ }^{35}$ E. Gorini, ${ }^{65 a, 65 b}$ A. Gorišek, ${ }^{89}$ A. T. Goshaw, ${ }^{47}$ C. Gössling, ${ }^{45}$ M. I. Gostkin,${ }^{77}$ C. A. Gottardo, ${ }^{24}$ C. R. Goudet, ${ }^{129}$ D. Goujdami, ${ }^{34 c}$ A. G. Goussiou, ${ }^{145}$ N. Govender, ${ }^{32 b, w}$ C. Goy, ${ }^{5}$ E. Gozani, ${ }^{157}$ I. Grabowska-Bold, ${ }^{81 a}$ P. O. J. Gradin, ${ }^{169}$ E. C. Graham, ${ }^{88}$ J. Gramling, ${ }^{168}$ E. Gramstad, ${ }^{131}$ S. Grancagnolo, ${ }^{19}$ V. Gratchev, ${ }^{135}$ P. M. Gravila, ${ }^{27 f}$ F. G. Gravili, ${ }^{65 a, 65 b}$ C. Gray, ${ }^{55}$ H. M. Gray, ${ }^{18}$ Z. D. Greenwood, ${ }^{93, x}$ C. Grefe, ${ }^{24}$ K. Gregersen, ${ }^{94}$ I. M. Gregor, ${ }^{44}$ P. Grenier, ${ }^{150}$ K. Grevtsov, ${ }^{44}$ N. A. Grieser, ${ }^{125}$ J. Griffiths, ${ }^{8}$ A. A. Grillo, ${ }^{143}$ K. Grimm, ${ }^{150, y}$ S. Grinstein, ${ }^{14, z}$ Ph. Gris, ${ }^{37}$ J.-F. Grivaz, ${ }^{129}$ S. Groh ${ }^{97}$ E. Gross, ${ }^{177}$ J. Grosse-Knetter, ${ }^{51}$ G. C. Grossi, ${ }^{93}$ Z. J. Grout, ${ }^{92}$ C. Grud, ${ }^{103}$ A. Grummer, ${ }^{116}$ L. Guan, ${ }^{103}$ W. Guan, ${ }^{178}$ J. Guenther, ${ }^{35}$ A. Guerguichon, ${ }^{129}$ F. Guescini,${ }^{165 a}$ D. Guest, ${ }^{168}$ R. Gugel, ${ }^{50}$ B. Gui, ${ }^{123}$ T. Guillemin,,${ }^{5}$ S. Guindon, ${ }^{35}$ U. Gul, ${ }^{55}$ J. Guo, ${ }^{58 \mathrm{c}}$ W. Guo, ${ }^{103}$ Y. Guo, ${ }^{58 a, a a}$ Z. Guo,${ }^{99}$ R. Gupta, ${ }^{44}$ S. Gurbuz, ${ }^{12 \mathrm{c}}$ G. Gustavino, ${ }^{125}$ P. Gutierrez, ${ }^{125}$ C. Gutschow, ${ }^{92}$ C. Guyot, ${ }^{142}$ M. P. Guzik, ${ }^{81 a}$ C. Gwenlan, ${ }^{132}$ C. B. Gwilliam, ${ }^{88}$ A. Haas, ${ }^{122}$ C. Haber ${ }^{18}$ H. K. Hadavand, ${ }^{8}$ N. Haddad, ${ }^{34 \mathrm{e}}$ A. Hadef, ${ }^{58 a}$ S. Hageböck, ${ }^{24}$ M. Hagihara, ${ }^{166}$ M. Haleem, ${ }^{174}$ J. Haley, ${ }^{126}$ G. Halladjian, ${ }^{104}$ G. D. Hallewell, ${ }^{99}$ 
K. Hamacher, ${ }^{179}$ P. Hamal, ${ }^{127}$ K. Hamano, ${ }^{173}$ A. Hamilton, ${ }^{32 \mathrm{a}}$ G. N. Hamity, ${ }^{146}$ K. Han,${ }^{58 a, b b}$ L. Han, ${ }^{58 \mathrm{a}}$ S. Han, ${ }^{15 \mathrm{~d}}$ K. Hanagaki, ${ }^{79, c c}$ M. Hance, ${ }^{143}$ D. M. Handl, ${ }^{112}$ B. Haney, ${ }^{134}$ R. Hankache, ${ }^{133}$ P. Hanke, ${ }^{59 a}$ E. Hansen, ${ }^{94}$ J. B. Hansen, ${ }^{39}$ J. D. Hansen, ${ }^{39}$ M. C. Hansen, ${ }^{24}$ P. H. Hansen, ${ }^{39}$ K. Hara, ${ }^{166}$ A. S. Hard,${ }^{178}$ T. Harenberg, ${ }^{179}$ S. Harkusha, ${ }^{105}$ P. F. Harrison, ${ }^{175}$ N. M. Hartmann, ${ }^{112}$ Y. Hasegawa, ${ }^{147}$ A. Hasib, ${ }^{48}$ S. Hassani, ${ }^{142}$ S. Haug, ${ }^{20}$ R. Hauser, ${ }^{104}$ L. Hauswald, ${ }^{46}$ L. B. Havener, ${ }^{38}$ M. Havranek, ${ }^{139}$ C. M. Hawkes, ${ }^{21}$ R. J. Hawkings, ${ }^{35}$ D. Hayden, ${ }^{104}$ C. Hayes, ${ }^{152}$ C. P. Hays, ${ }^{132}$ J. M. Hays, ${ }^{90}$ H. S. Hayward, ${ }^{88}$ S. J. Haywood ${ }^{141}$ F. He, ${ }^{58 a}$ M. P. Heath, ${ }^{48}$ V. Hedberg, ${ }^{94}$ L. Heelan, ${ }^{8}$ S. Heer, ${ }^{24}$ K. K. Heidegger,${ }^{50}$ J. Heilman, ${ }^{33}$ S. Heim,,${ }^{44}$ T. Heim, ${ }^{18}$ B. Heinemann, ${ }^{44, d d}$ J. J. Heinrich, ${ }^{112}$ L. Heinrich, ${ }^{122}$ C. Heinz, ${ }^{54}$ J. Hejbal, ${ }^{138}$ L. Helary, ${ }^{35}$ A. Held ${ }^{172}$ S. Hellesund, ${ }^{131}$ C. M. Helling, ${ }^{143}$ S. Hellman, ${ }^{43 a, 43 b}$ C. Helsens,${ }^{35}$ R. C. W. Henderson, ${ }^{87}$ Y. Heng, ${ }^{178}$ S. Henkelmann, ${ }^{172}$ A. M. Henriques Correia, ${ }^{35}$ G. H. Herbert, ${ }^{19}$ H. Herde, ${ }^{26}$ V. Herget, ${ }^{174}$ Y. Hernández Jiménez, ${ }^{32 \mathrm{c}}$ H. Herr, ${ }^{97}$ M. G. Herrmann, ${ }^{112}$ T. Herrmann, ${ }^{46}$ G. Herten, ${ }^{50}$ R. Hertenberger, ${ }^{112}$ L. Hervas, ${ }^{35}$ T. C. Herwig, ${ }^{134}$ G. G. Hesketh, ${ }^{92}$ N. P. Hessey, ${ }^{165 a}$ A. Higashida, ${ }^{160}$ S. Higashino, ${ }^{79}$ E. Higón-Rodriguez, ${ }^{171}$ K. Hildebrand, ${ }^{36}$ E. Hill, ${ }^{173}$ J. C. Hill, ${ }^{31}$ K. K. Hill, ${ }^{29}$ K. H. Hiller, ${ }^{44}$ S. J. Hillier, ${ }^{21}$ M. Hils, ${ }^{46}$ I. Hinchliffe, ${ }^{18}$ F. Hinterkeuser, ${ }^{24}$ M. Hirose, ${ }^{130}$ D. Hirschbuehl, ${ }^{179}$ B. Hiti, ${ }^{89}$ O. Hladik, ${ }^{138}$ D. R. Hlaluku, ${ }^{32 c}$ X. Hoad, ${ }^{48}$ J. Hobbs, ${ }^{152}$ N. Hod, ${ }^{165 a}$ M. C. Hodgkinson, ${ }^{146}$ A. Hoecker, ${ }^{35}$ M. R. Hoeferkamp, ${ }^{116}$ F. Hoenig, ${ }^{112}$ D. Hohn,${ }^{50}$ D. Hohov, ${ }^{129}$ T. R. Holmes, ${ }^{36}$ M. Holzbock, ${ }^{112}$ M. Homann, ${ }^{45}$ B. H. Hommels, ${ }^{31}$ S. Honda, ${ }^{166}$ T. Honda,${ }^{79}$ T. M. Hong, ${ }^{136}$ A. Hönle, ${ }^{113}$ B. H. Hooberman, ${ }^{170}$ W. H. Hopkins, ${ }^{128}$ Y. Horii, ${ }^{115}$ P. Horn, ${ }^{46}$ A. J. Horton, ${ }^{149}$ L. A. Horyn, ${ }^{36}$ J-Y. Hostachy, ${ }^{56}$ A. Hostiuc, ${ }^{145}$ S. Hou, ${ }^{155}$ A. Hoummada, ${ }^{34 a}$ J. Howarth, ${ }^{98}$ J. Hoya ${ }^{86}$ M. Hrabovsky, ${ }^{127}$ J. Hrdinka,${ }^{35}$ I. Hristova, ${ }^{19}$ J. Hrivnac, ${ }^{129}$ A. Hrynevich, ${ }^{106}$ T. Hryn'ova, ${ }^{5}$ P. J. Hsu, ${ }^{62}$ S.-C. Hsu, ${ }^{145}$ Q. Hu, ${ }^{29}$ S. Hu, ${ }^{58 c}$ Y. Huang, ${ }^{15 a}$ Z. Hubacek, ${ }^{139}$ F. Hubaut, ${ }^{99}$ M. Huebner, ${ }^{24}$ F. Huegging, ${ }^{24}$ T. B. Huffman, ${ }^{132}$ M. Huhtinen, ${ }^{35}$ R. F. H. Hunter, ${ }^{33}$ P. Huo, ${ }^{152}$ A. M. Hupe, ${ }^{33}$ N. Huseynov, ${ }^{77, \mathrm{e}}$ J. Huston, ${ }^{104}$ J. Huth, ${ }^{57}$ R. Hyneman, ${ }^{103}$ G. Iacobucci, ${ }^{52}$ G. Iakovidis, ${ }^{29}$ I. Ibragimov, ${ }^{148}$ L. Iconomidou-Fayard, ${ }^{129}$ Z. Idrissi, ${ }^{34 \mathrm{e}}$ P. Iengo, ${ }^{35}$ R. Ignazzi, ${ }^{39}$ O. Igonkina, ${ }^{18, \text { ee }}$ R. Iguchi, ${ }^{160}$ T. Iizawa, ${ }^{52}$ Y. Ikegami, ${ }^{79} \mathrm{M}$. Ikeno, ${ }^{79} \mathrm{D}$. Iliadis, ${ }^{159} \mathrm{~N}$. Ilic, ${ }^{117} \mathrm{~F}$. Iltzsche, ${ }^{46} \mathrm{G}$. Introzzi,${ }^{68 a, 68 \mathrm{~b}}$ M. Iodice, ${ }^{72 \mathrm{a}} \mathrm{K}$. Iordanidou, ${ }^{38} \mathrm{~V}$. Ippolito, ${ }^{70 a, 70 \mathrm{~b}} \mathrm{M}$. F. Isacson, ${ }^{169} \mathrm{~N}$. Ishijima, ${ }^{130} \mathrm{M}$. Ishino, ${ }^{160} \mathrm{M}$. Ishitsuka, ${ }^{162} \mathrm{~W}$. Islam, ${ }^{126}$ C. Issever, ${ }^{132} \mathrm{~S}$. Istin, ${ }^{157} \mathrm{~F}$. Ito, ${ }^{166} \mathrm{~J}$. M. Iturbe Ponce, ${ }^{61 \mathrm{a}} \mathrm{R}$. Iuppa, ${ }^{73 a, 73 \mathrm{~b}} \mathrm{~A}$. Ivina, ${ }^{177} \mathrm{H}$. Iwasaki, ${ }^{79} \mathrm{~J}$. M. Izen, ${ }^{42} \mathrm{~V}$. Izzo, ${ }^{67 \mathrm{a}}$ P. Jacka, ${ }^{138}$ P. Jackson, ${ }^{1}$ R. M. Jacobs,${ }^{24}$ V. Jain, ${ }^{2}$ G. Jäkel, ${ }^{179}$ K. B. Jakobi, ${ }^{97}$ K. Jakobs,${ }^{50}$ S. Jakobsen, ${ }^{74}$ T. Jakoubek, ${ }^{138}$ D. O. Jamin, ${ }^{126}$ R. Jansky, ${ }^{52}$ J. Janssen, ${ }^{24}$ M. Janus, ${ }^{51}$ P. A. Janus, ${ }^{81 a}$ G. Jarlskog, ${ }^{94}$ N. Javadov, ${ }^{77, e}$ T. Javưrek, ${ }^{35}$ M. Javurkova, ${ }^{50}$ F. Jeanneau, ${ }^{142}$ L. Jeanty, ${ }^{18}$ J. Jejelava, ${ }^{156 a, f f}$ A. Jelinskas, ${ }^{175}$ P. Jenni, ${ }^{50, g g}$ J. Jeong, ${ }^{44}$ N. Jeong, ${ }^{44}$ S. Jézéquel, ${ }^{5}$ H. Ji, ${ }^{178}$ J. Jia, ${ }^{152}$ H. Jiang, ${ }^{76}$ Y. Jiang,${ }^{58 a}$ Z. Jiang, ${ }^{150, h h}$ S. Jiggins,${ }^{50}$ F. A. Jimenez Morales, ${ }^{37}$ J. Jimenez Pena, ${ }^{171}$ S. Jin, ${ }^{15 c}$ A. Jinaru, ${ }^{27 b}$ O. Jinnouchi, ${ }^{162}$ H. Jivan, ${ }^{32 c}$ P. Johansson, ${ }^{146}$ K. A. Johns, ${ }^{7}$ C. A. Johnson, ${ }^{63}$ K. Jon-And, ${ }^{43 a, 43 b}$ R. W. L. Jones, ${ }^{87}$ S. D. Jones, ${ }^{153}$ S. Jones, ${ }^{7}$ T. J. Jones, ${ }^{88}$ J. Jongmanns, ${ }^{59 a}$ P. M. Jorge, ${ }^{137 a, 137 b}$ J. Jovicevic, ${ }^{165 a}$ X. Ju, ${ }^{18}$ J. J. Junggeburth, ${ }^{113}$ A. Juste Rozas, ${ }^{14, z}$ A. Kaczmarska, ${ }^{82}$ M. Kado, ${ }^{129}$ H. Kagan, ${ }^{123}$ M. Kagan, ${ }^{150}$ T. Kaji, ${ }^{176}$ E. Kajomovitz, ${ }^{157}$ C. W. Kalderon, ${ }^{94}$ A. Kaluza, ${ }^{97}$ S. Kama, ${ }^{41}$ A. Kamenshchikov, ${ }^{121}$ L. Kanjir, ${ }^{89}$ Y. Kano, ${ }^{160}$ V. A. Kantserov, ${ }^{110}$ J. Kanzaki, ${ }^{79}$ L. S. Kaplan, ${ }^{178}$ D. Kar, ${ }^{32 \mathrm{c}}$ M. J. Kareem, ${ }^{165 \mathrm{~b}}$ E. Karentzos, ${ }^{10}$ S. N. Karpov, ${ }^{77}$ Z. M. Karpova, ${ }^{77}$ V. Kartvelishvili, ${ }^{87}$ A. N. Karyukhin, ${ }^{111}$ L. Kashif, ${ }^{178}$ R. D. Kass, ${ }^{123}$ A. Kastanas, ${ }^{43 a, 43 b}$ Y. Kataoka, ${ }^{160}$ C. Kato, ${ }^{58 d, 58 c}$ J. Katzy, ${ }^{44}$ K. Kawade,${ }^{80}$ K. Kawagoe, ${ }^{85}$ T. Kawaguchi, ${ }^{115}$ T. Kawamoto, ${ }^{160}$ G. Kawamura, ${ }^{51}$ E. F. Kay ${ }^{88}$ V. F. Kazanin, ${ }^{120 b, 120 a}$ R. Keeler, ${ }^{173}$ R. Kehoe, ${ }^{41}$ J. S. Keller, ${ }^{33}$ E. Kellermann,,${ }^{94}$ J. J. Kempster, ${ }^{21}$ J. Kendrick,${ }^{21}$ O. Kepka, ${ }^{138}$ S. Kersten, ${ }^{179}$ B. P. Kerševan ${ }^{89}$ S. Ketabchi Haghighat, ${ }^{164}$ R. A. Keyes, ${ }^{101}$ M. Khader, ${ }^{170}$ F. Khalil-Zada, ${ }^{13}$ A. Khanov, ${ }^{126}$

A. G. Kharlamov, ${ }^{120 b, 120 a}$ T. Kharlamova, ${ }^{120 b, 120 a}$ E. E. Khoda, ${ }^{172}$ A. Khodinov, ${ }^{163}$ T. J. Khoo, ${ }^{52}$ E. Khramov, ${ }^{77}$

J. Khubua, ${ }^{156 \mathrm{~b}}$ S. Kido, ${ }^{80}$ M. Kiehn, ${ }^{52}$ C. R. Kilby, ${ }^{91}$ Y. K. Kim, ${ }^{36}$ N. Kimura, ${ }^{64 \mathrm{a}, 64 \mathrm{c}}$ O. M. Kind, ${ }^{19}$ B. T. King, ${ }^{88}$ D. Kirchmeier, ${ }^{46}$ J. Kirk, ${ }^{141}$ A. E. Kiryunin, ${ }^{113}$ T. Kishimoto, ${ }^{160}$ D. Kisielewska, ${ }^{81 a}$ V. Kitali, ${ }^{44}$ O. Kivernyk, ${ }^{5}$ E. Kladiva, ${ }^{28 b, a}$ T. Klapdor-Kleingrothaus, ${ }^{50}$ M. H. Klein, ${ }^{103}$ M. Klein ${ }^{88}$ U. Klein,${ }^{88}$ K. Kleinknecht, ${ }^{97}$ P. Klimek, ${ }^{119}$ A. Klimentov, ${ }^{29}$ T. Klingl, ${ }^{24}$ T. Klioutchnikova, ${ }^{35}$ F. F. Klitzner, ${ }^{112}$ P. Kluit, ${ }^{118}$ S. Kluth, ${ }^{113}$ E. Kneringer, ${ }^{74}$ E. B. F. G. Knoops, ${ }^{99}$ A. Knue, ${ }^{50}$ A. Kobayashi, ${ }^{160}$ D. Kobayashi, ${ }^{85}$ T. Kobayashi, ${ }^{160}$ M. Kobel, ${ }^{46}$ M. Kocian, ${ }^{150}$ P. Kodys, ${ }^{140}$ P. T. Koenig, ${ }^{24}$ T. Koffas, ${ }^{33}$ E. Koffeman, ${ }^{118}$ N. M. Köhler, ${ }^{113}$ T. Koi, ${ }^{150}$ M. Kolb, ${ }^{59 b}$ I. Koletsou, ${ }^{5}$ T. Kondo, ${ }^{79}$ N. Kondrashova, ${ }^{58 c}$ K. Köneke, ${ }^{50}$ A. C. König, ${ }^{117}$ T. Kono, ${ }^{79}$ R. Konoplich, ${ }^{122, i i}$ V. Konstantinides, ${ }^{92}$ N. Konstantinidis, ${ }^{92}$ B. Konya,${ }^{94}$ R. Kopeliansky, ${ }^{63}$ S. Koperny, ${ }^{81 a}$ K. Korcyl, ${ }^{82}$ K. Kordas, ${ }^{159}$ G. Koren, ${ }^{158}$ A. Korn, ${ }^{92}$ I. Korolkov, ${ }^{14}$ E. V. Korolkova, ${ }^{146}$ N. Korotkova, ${ }^{111}$ O. Kortner, ${ }^{113}$ S. Kortner, ${ }^{113}$ T. Kosek, ${ }^{140}$ V. V. Kostyukhin, ${ }^{24}$ A. Kotwal,${ }^{47}$ A. Koulouris, ${ }^{10}$

A. Kourkoumeli-Charalampidi, ${ }^{68,68 b}$ C. Kourkoumelis, ${ }^{9}$ E. Kourlitis, ${ }^{146}$ V. Kouskoura, ${ }^{29}$ A. B. Kowalewska, ${ }^{82}$ R. Kowalewski, ${ }^{173}$ T. Z. Kowalski, ${ }^{81 a}$ C. Kozakai, ${ }^{160}$ W. Kozanecki, ${ }^{142}$ A. S. Kozhin, ${ }^{121}$ V. A. Kramarenko, ${ }^{111}$ G. Kramberger, ${ }^{89}$ D. Krasnopevtsev, ${ }^{58 \mathrm{a}}$ M. W. Krasny, ${ }^{133}$ A. Krasznahorkay, ${ }^{35}$ D. Krauss, ${ }^{113}$ J. A. Kremer, ${ }^{81 a}$ 
J. Kretzschmar, ${ }^{88}$ P. Krieger, ${ }^{164}$ K. Krizka, ${ }^{18}$ K. Kroeninger, ${ }^{45}$ H. Kroha, ${ }^{113}$ J. Kroll, ${ }^{138}$ J. Kroll,,${ }^{134}$ J. Krstic, ${ }^{16}$ U. Kruchonak, ${ }^{77}$ H. Krüger, ${ }^{24}$ N. Krumnack, ${ }^{76}$ M. C. Kruse, ${ }^{47}$ T. Kubota, ${ }^{102}$ S. Kuday, ${ }^{4 b}$ J. T. Kuechler, ${ }^{179}$ S. Kuehn, ${ }^{35}$ A. Kugel, ${ }^{59 a}$ T. Kuhl, ${ }^{44}$ V. Kukhtin, ${ }^{77}$ R. Kukla, ${ }^{99}$ Y. Kulchitsky, ${ }^{105, j j}$ S. Kuleshov, ${ }^{14 b}$ Y. P. Kulinich, ${ }^{170}$ M. Kuna, ${ }^{56}$ T. Kunigo, ${ }^{83}$ A. Kupco, ${ }^{138}$ T. Kupfer, ${ }^{45}$ O. Kuprash, ${ }^{158}$ H. Kurashige, ${ }^{80}$ L. L. Kurchaninov, ${ }^{165 a}$ Y. A. Kurochkin, ${ }^{105}$ A. Kurova, ${ }^{110}$ M. G. Kurth, ${ }^{15 \mathrm{~d}}$ E. S. Kuwertz, ${ }^{35}$ M. Kuze, ${ }^{162}$ J. Kvita, ${ }^{127}$ T. Kwan, ${ }^{101}$ A. La Rosa, ${ }^{113}$ J. L. La Rosa Navarro, ${ }^{78 d}$ L. La Rotonda,${ }^{40 b, 40 a}$ F. La Ruffa, ${ }^{40 b, 40 a}$ C. Lacasta, ${ }^{171}$ F. Lacava, ${ }^{70 a, 70 b}$ J. Lacey ${ }^{44}$ D. P. J. Lack, ${ }^{98}$ H. Lacker, ${ }^{19}$ D. Lacour, ${ }^{133}$ E. Ladygin, ${ }^{77}$ R. Lafaye, ${ }^{5}$ B. Laforge, ${ }^{133}$ T. Lagouri, ${ }^{32 c}$ S. Lai,${ }^{51}$ S. Lammers, ${ }^{63}$ W. Lampl, ${ }^{7}$ E. Lançon, ${ }^{29}$ U. Landgraf,${ }^{50}$ M. P. J. Landon, ${ }^{90}$ M. C. Lanfermann, ${ }^{52}$ V. S. Lang, ${ }^{44}$ J. C. Lange, ${ }^{51}$ R. J. Langenberg, ${ }^{35}$ A. J. Lankford, ${ }^{168}$ F. Lanni, ${ }^{29}$

K. Lantzsch ${ }^{24}$ A. Lanza, ${ }^{68 a}$ A. Lapertosa, ${ }^{53 b, 53 a}$ S. Laplace, ${ }^{133}$ J. F. Laporte, ${ }^{142}$ T. Lari, ${ }^{66 a}$ F. Lasagni Manghi, ${ }^{23 b, 23 a}$

M. Lassnig, ${ }^{35}$ T. S. Lau, ${ }^{61 \mathrm{a}}$ A. Laudrain, ${ }^{129}$ M. Lavorgna, ${ }^{67 \mathrm{a}, 67 \mathrm{~b}}$ M. Lazzaroni, ${ }^{66 a, 66 \mathrm{~b}}$ B. Le, ${ }^{102}$ O. Le Dortz, ${ }^{133}$

E. Le Guirriec, ${ }^{99}$ E. P. Le Quilleuc, ${ }^{142}$ M. LeBlanc, ${ }^{7}$ T. LeCompte, ${ }^{6}$ F. Ledroit-Guillon, ${ }^{56}$ C. A. Lee, ${ }^{29}$ G. R. Lee, ${ }^{144 a}$ L. Lee,${ }^{57}$ S. C. Lee, ${ }^{155}$ B. Lefebvre, ${ }^{101}$ M. Lefebvre, ${ }^{173}$ F. Legger, ${ }^{12}$ C. Leggett, ${ }^{18}$ K. Lehmann, ${ }^{149}$ N. Lehmann, ${ }^{179}$ G. Lehmann Miotto, ${ }^{35}$ W. A. Leight, ${ }^{44}$ A. Leisos, ${ }^{159, k \mathrm{kk}}$ M. A. L. Leite, ${ }^{78 \mathrm{~d}}$ R. Leitner, ${ }^{140}$ D. Lellouch, ${ }^{177}$ K. J. C. Leney, ${ }^{92}$ T. Lenz,${ }^{24}$ B. Lenzi, ${ }^{35}$ R. Leone,${ }^{7}$ S. Leone, ${ }^{69 a}$ C. Leonidopoulos, ${ }^{48}$ G. Lerner, ${ }^{153}$ C. Leroy, ${ }^{107}$ R. Les, ${ }^{164}$ A. A. J. Lesage, ${ }^{142}$ C. G. Lester, ${ }^{31}$ M. Levchenko, ${ }^{135}$ J. Levêque, ${ }^{5}$ D. Levin, ${ }^{103}$ L. J. Levinson, ${ }^{177}$ D. Lewis, ${ }^{90}$ B. Li, ${ }^{15 b}$ B. Li,${ }^{103}$ C-Q. Li,${ }^{58 a, 11}$ H. Li, ${ }^{58 \mathrm{a}} \mathrm{H} . \mathrm{Li}^{58 \mathrm{~b}}{ }^{\mathrm{K}} \mathrm{Li},{ }^{150} \mathrm{~L} . \mathrm{Li},{ }^{58 \mathrm{c}} \mathrm{M} . \mathrm{Li},{ }^{15 \mathrm{a}} \mathrm{Q} . \mathrm{Li},{ }^{15 \mathrm{~d}} \mathrm{Q}$. Y. Li ${ }^{58 \mathrm{a}} \mathrm{S} . \mathrm{Li},{ }^{58 \mathrm{~d}, 58 \mathrm{c}}$ X. Li, ${ }^{58 \mathrm{c}} \mathrm{Y} . \mathrm{Li},{ }^{148}$ Z. Liang, ${ }^{15 \mathrm{a}}$ B. Liberti, ${ }^{71 \mathrm{a}}$ A. Liblong, ${ }^{164}$ K. Lie, ${ }^{61 \mathrm{c}}$ S. Liem, ${ }^{118}$ A. Limosani, ${ }^{154}$ C. Y. Lin, ${ }^{31}$ K. Lin,${ }^{104}$ T. H. Lin, ${ }^{97}$ R. A. Linck, ${ }^{63}$ J. H. Lindon, ${ }^{21}$ B. E. Lindquist, ${ }^{152}$ A. L. Lionti, ${ }^{52}$ E. Lipeles, ${ }^{134}$ A. Lipniacka, ${ }^{17}$ M. Lisovyi,${ }^{59 b}$ T. M. Liss, ${ }^{170, m m}$ A. Lister, ${ }^{172}$ A. M. Litke, ${ }^{143}$ J. D. Little, ${ }^{8}$ B. Liu, ${ }^{76}$ B. L Liu, ${ }^{6}$ H. B. Liu ${ }^{29}$ H. Liu,${ }^{103}$ J. B. Liu, ${ }^{58 a}$ J. K. K. Liu, ${ }^{132}$ K. Liu, ${ }^{133}$ M. Liu, ${ }^{58 a}$ P. Liu, ${ }^{18}$ Y. Liu, ${ }^{15 a}$ Y. L. Liu, ${ }^{58 \mathrm{a}}$ Y. W. Liu, ${ }^{58 \mathrm{a}}$ M. Livan, ${ }^{68 \mathrm{a}, 68 \mathrm{~b}}$ A. Lleres,${ }^{56}$ J. Llorente Merino, ${ }^{15 \mathrm{a}}$ S. L. Lloyd ${ }^{90}$ C. Y. Lo, ${ }^{61 b}$ F. Lo Sterzo, ${ }^{41}$ E. M. Lobodzinska, ${ }^{44}$ P. Loch, ${ }^{7}$ T. Lohse,${ }^{19}$ K. Lohwasser,${ }^{146}$ M. Lokajicek, ${ }^{138}$ J. D. Long, ${ }^{170}$ R. E. Long, ${ }^{87}$ L. Longo, ${ }^{65 a, 65 b}$ K. A. Looper, ${ }^{123}$ J. A. Lopez, ${ }^{144 b}$ I. Lopez Paz, ${ }^{98}$ A. Lopez Solis, ${ }^{146}$ J. Lorenz, ${ }^{112}$ N. Lorenzo Martinez,${ }^{5}$ M. Losada, ${ }^{22}$ P. J. Lösel, ${ }^{112}$ A. Lösle, ${ }^{50}$ X. Lou ${ }^{44}$ X. Lou, ${ }^{15 a}$ A. Lounis,${ }^{129}$ J. Love, ${ }^{6}$ P. A. Love ${ }^{87}$ J. J. Lozano Bahilo, ${ }^{171}$ H. Lu, ${ }^{61 a}$ M. Lu, ${ }^{58 a}$ Y. J. Lu, ${ }^{62}$ H. J. Lubatti, ${ }^{145}$ C. Luci ${ }^{70 a, 70 b}$ A. Lucotte, ${ }^{56}$ C. Luedtke, ${ }^{50}$ F. Luehring, ${ }^{63}$ I. Luise,${ }^{133}$ L. Luminari, ${ }^{70 a}$ B. Lund-Jensen, ${ }^{151}$ M. S. Lutz, ${ }^{100}$ P. M. Luzi, ${ }^{133}$ D. Lynn, ${ }^{29}$ R. Lysak, ${ }^{138}$ E. Lytken, ${ }^{94}$ F. Lyu, ${ }^{15 a}$ V. Lyubushkin, ${ }^{77}$ T. Lyubushkina, ${ }^{77}$ H. Ma, ${ }^{29}$ L. L. Ma, ${ }^{58 b}$ Y. Ma, ${ }^{58 b}$ G. Maccarrone, ${ }^{49}$ A. Macchiolo, ${ }^{113}$ C. M. Macdonald, ${ }^{146}$ J. Machado Miguens, ${ }^{134,137 b}$ D. Madaffari, ${ }^{171}$ R. Madar, ${ }^{37}$ W. F. Mader, ${ }^{46}$ N. Madysa, ${ }^{46}$ J. Maeda, ${ }^{80}$ K. Maekawa, ${ }^{160}$ S. Maeland ${ }^{17}$ T. Maeno, ${ }^{29}$ M. Maerker, ${ }^{46}$ A. S. Maevskiy, ${ }^{111}$ V. Magerl, ${ }^{50}$ D. J. Mahon,${ }^{38}$ C. Maidantchik, ${ }^{78 b}$ T. Maier, ${ }^{112}$ A. Maio, ${ }^{137 a, 137 b, 137 d}$ O. Majersky, ${ }^{28 a}$ S. Majewski, ${ }^{128}$ Y. Makida,${ }^{79}$ N. Makovec, ${ }^{129}$ B. Malaescu, ${ }^{133}$ Pa. Malecki ${ }^{82}$ V. P. Maleev, ${ }^{135}$ F. Malek, ${ }^{56}$ U. Mallik ${ }^{75}$ D. Malon, ${ }^{6}$ C. Malone, ${ }^{31}$ S. Maltezos,${ }^{10}$ S. Malyukov, ${ }^{35}$ J. Mamuzic, ${ }^{171}$ G. Mancini ${ }^{49}$ I. Mandic ${ }^{89}$ J. Maneira, ${ }^{137 a}$ L. Manhaes de Andrade Filho, ${ }^{78 a}$ J. Manjarres Ramos, ${ }^{46}$ K. H. Mankinen, ${ }^{94}$ A. Mann, ${ }^{112}$ A. Manousos, ${ }^{74}$ B. Mansoulie, ${ }^{142}$ S. Manzoni, ${ }^{66 a, 66 b}$ A. Marantis, ${ }^{159}$ G. Marceca, ${ }^{30}$ L. March, ${ }^{52}$ L. Marchese, ${ }^{132}$ G. Marchiori, ${ }^{133}$ M. Marcisovsky, ${ }^{138}$ C. Marcon, ${ }^{94}$ C. A. Marin Tobon, ${ }^{35}$ M. Marjanovic, ${ }^{37}$ F. Marroquim, ${ }^{78 b}$ Z. Marshall, ${ }^{18}$ M. U. F Martensson, ${ }^{169}$ S. Marti-Garcia, ${ }^{171}$ C. B. Martin, ${ }^{123}$ T. A. Martin, ${ }^{175}$ V. J. Martin, ${ }^{48}$ B. Martin dit Latour, ${ }^{17}$ M. Martinez, ${ }^{14, z}$ V. I. Martinez Outschoorn, ${ }^{100}$ S. Martin-Haugh, ${ }^{141}$ V. S. Martoiu, ${ }^{27 b}$ A. C. Martyniuk, ${ }^{92}$ A. Marzin, ${ }^{35}$ L. Masetti, ${ }^{97}$ T. Mashimo, ${ }^{160}$ R. Mashinistov, ${ }^{108}$ J. Masik, ${ }^{98}$ A. L. Maslennikov, ${ }^{120 b, 120 a}$ L. H. Mason, ${ }^{102}$ L. Massa, ${ }^{71,71 b}$ P. Massarotti, ${ }^{67 a, 67 b}$ P. Mastrandrea, ${ }^{152}$ A. Mastroberardino, ${ }^{40 b, 40 \mathrm{a}}$ T. Masubuchi, ${ }^{160}$ P. Mättig, ${ }^{24} \mathrm{~J}$. Maurer, ${ }^{27 \mathrm{~b}}$ B. Maček ${ }^{89}$ S. J. Maxfield, ${ }^{88}$ D. A. Maximov, ${ }^{120 b, 120 a}$ R. Mazini, ${ }^{155}$ I. Maznas, ${ }^{159}$ S. M. Mazza, ${ }^{143}$ S. P. Mc Kee, ${ }^{103}$ A. McCarn, ${ }^{41}$ T. G. McCarthy, ${ }^{113}$ L. I. McClymont, ${ }^{92}$ W. P. McCormack, ${ }^{18}$ E. F. McDonald, ${ }^{102}$ J. A. Mcfayden,${ }^{35}$ G. Mchedlidze, ${ }^{51}$ M. A. McKay, ${ }^{41}$ K. D. McLean, ${ }^{173}$ S. J. McMahon, ${ }^{141}$ P. C. McNamara, ${ }^{102}$ C. J. McNicol ${ }^{175}$ R. A. McPherson, ${ }^{173,0}$ J. E. Mdhluli, ${ }^{32 \mathrm{c}}$ Z. A. Meadows, ${ }^{100}$ S. Meehan, ${ }^{145}$ T. M. Megy, ${ }^{50}$ S. Mehlhase, ${ }^{112}$ A. Mehta ${ }^{88}$ T. Meideck, ${ }^{56}$ B. Meirose,${ }^{42}$ D. Melini, ${ }^{171, n n}$ B. R. Mellado Garcia, ${ }^{32 c}$ J. D. Mellenthin, ${ }^{51}$ M. Melo, ${ }^{28 a}$ F. Meloni, ${ }^{44}$ A. Melzer, ${ }^{24}$ S. B. Menary, ${ }^{98}$ E. D. Mendes Gouveia, ${ }^{137 a}$ L. Meng, ${ }^{88}$ X. T. Meng, ${ }^{103}$ S. Menke, ${ }^{113}$ E. Meoni, ${ }^{40 b, 40 a}$ S. Mergelmeyer, ${ }^{19}$ S. A. M. Merkt, ${ }^{136}$ C. Merlassino, ${ }^{20}$ P. Mermod, ${ }^{52}$ L. Merola,${ }^{67,67 b}$ C. Meroni, ${ }^{66 a}$ F. S. Merritt, ${ }^{36}$ A. Messina, ${ }^{70 a, 70 b}$ J. Metcalfe, ${ }^{6}$ A. S. Mete, ${ }^{168}$ C. Meyer ${ }^{63}$ J. Meyer ${ }^{157}$ J-P. Meyer, ${ }^{142}$ H. Meyer Zu Theenhausen, ${ }^{59 a}$ F. Miano, ${ }^{153}$ R. P. Middleton, ${ }^{141}$ L. Mijović, ${ }^{48}$ G. Mikenberg, ${ }^{177}$ M. Mikestikova, ${ }^{138}$ M. Mikuž ${ }^{89}$ M. Milesi, ${ }^{102}$ A. Milic, ${ }^{164}$ D. A. Millar, ${ }^{90}$ D. W. Miller, ${ }^{36}$ A. Milov, ${ }^{177}$ D. A. Milstead ${ }^{43 a, 43 b}$ R. A. Mina, ${ }^{150, \text { hh }}$ A. A. Minaenko, ${ }^{121}$ M. Miñano Moya, ${ }^{171}$ I. A. Minashvili, ${ }^{156 b}$ A. I. Mincer, ${ }^{122}$ B. Mindur, ${ }^{81 a}$ M. Mineev, ${ }^{77}$ Y. Minegishi, ${ }^{160}$ Y. Ming,${ }^{178}$ L. M. Mir, ${ }^{14}$ A. Mirto ${ }^{65 a, 65 b}$ K. P. Mistry, ${ }^{134}$ T. Mitani, ${ }^{176}$ J. Mitrevski, ${ }^{112}$ V. A. Mitsou, ${ }^{171}$ M. Mittal, ${ }^{58 c}$ A. Miucci, ${ }^{20}$ P. S. Miyagawa, ${ }^{146}$ A. Mizukami, ${ }^{79}$ J. U. Mjörnmark, ${ }^{94}$ 
T. Mkrtchyan, ${ }^{181}$ M. Mlynarikova, ${ }^{140}$ T. Moa ${ }^{43 a, 43 b}$ K. Mochizuki, ${ }^{107}$ P. Mogg, ${ }^{50}$ S. Mohapatra, ${ }^{38}$ S. Molander ${ }^{43 a, 43 b}$ R. Moles-Valls, ${ }^{24}$ M. C. Mondragon, ${ }^{104}$ K. Mönig, ${ }^{44}$ J. Monk,${ }^{39}$ E. Monnier, ${ }^{99}$ A. Montalbano, ${ }^{149}$ J. Montejo Berlingen, ${ }^{35}$ F. Monticelli, ${ }^{86}$ S. Monzani ${ }^{66 a}$ N. Morange, ${ }^{129}$ D. Moreno, ${ }^{22}$ M. Moreno Llácer, ${ }^{35}$ P. Morettini, ${ }^{53 b}$ M. Morgenstern, ${ }^{118}$ S. Morgenstern, ${ }^{46}$ D. Mori ${ }^{149}$ M. Morii, ${ }^{57}$ M. Morinaga, ${ }^{176}$ V. Morisbak, ${ }^{131}$ A. K. Morley, ${ }^{35}$ G. Mornacchi, ${ }^{35}$ A. P. Morris,${ }^{92}$ J. D. Morris, ${ }^{90}$ L. Morvaj, ${ }^{152}$ P. Moschovakos, ${ }^{10}$ M. Mosidze, ${ }^{156 b}$ H. J. Moss, ${ }^{146}$ J. Moss, ${ }^{150, o 0}$ K. Motohashi, ${ }^{162}$ R. Mount, ${ }^{150}$ E. Mountricha, ${ }^{35}$ E. J. W. Moyse, ${ }^{100}$ S. Muanza, ${ }^{99}$ F. Mueller, ${ }^{113}$ J. Mueller, ${ }^{136}$ R. S. P. Mueller, ${ }^{12}$ D. Muenstermann, ${ }^{87}$ G. A. Mullier, ${ }^{94}$ F. J. Munoz Sanchez,${ }^{98}$ P. Murin,${ }^{28 b}$ W. J. Murray,${ }^{175,141}$ A. Murrone, ${ }^{66 a, 66 b}$ M. Muškinja,${ }^{89}$ C. Mwewa, ${ }^{32 a}$ A. G. Myagkov, ${ }^{121, p p}$ J. Myers, ${ }^{128}$ M. Myska, ${ }^{139}$ B. P. Nachman, ${ }^{18}$ O. Nackenhorst ${ }^{45}$ K. Nagai, ${ }^{132}$ K. Nagano, ${ }^{79}$ Y. Nagasaka, ${ }^{60}$ M. Nagel, ${ }^{50}$ E. Nagy, ${ }^{99}$ A. M. Nairz,${ }^{35}$ Y. Nakahama, ${ }^{115}$ K. Nakamura ${ }^{79}$ T. Nakamura ${ }^{160}$ I. Nakano, ${ }^{124}$ H. Nanjo, ${ }^{130}$ F. Napolitano, ${ }^{59 a}$ R. F. Naranjo Garcia, ${ }^{44}$ R. Narayan, ${ }^{11}$ D. I. Narrias Villar, ${ }^{59 a}$ I. Naryshkin, ${ }^{135}$ T. Naumann, ${ }^{44}$ G. Navarro, ${ }^{22}$ R. Nayyar, ${ }^{7}$ H. A. Neal, ${ }^{103, a}$ P. Y. Nechaeva, ${ }^{108}$ T. J. Neep, ${ }^{142}$ A. Negri, ${ }^{68 a, 68 b}$ M. Negrini, ${ }^{23 b}$ S. Nektarijevic, ${ }^{117}$

C. Nellist, ${ }^{51}$ M. E. Nelson, ${ }^{132}$ S. Nemecek, ${ }^{138}$ P. Nemethy, ${ }^{122}$ M. Nessi,,${ }^{35, q q}$ M. S. Neubauer, ${ }^{170}$ M. Neumann, ${ }^{179}$

P. R. Newman, ${ }^{21}$ T. Y. Ng, ${ }^{61 \mathrm{c}}$ Y. S. Ng, ${ }^{19}$ Y. W. Y. Ng, ${ }^{168}$ H. D. N. Nguyen, ${ }^{99}$ T. Nguyen Manh, ${ }^{107}$ E. Nibigira, ${ }^{37}$ R. B. Nickerson, ${ }^{132}$ R. Nicolaidou, ${ }^{142}$ D. S. Nielsen, ${ }^{39}$ J. Nielsen, ${ }^{143}$ N. Nikiforou, ${ }^{11}$ V. Nikolaenko, ${ }^{121, p p}$ I. Nikolic-Audit, ${ }^{133}$ K. Nikolopoulos, ${ }^{21}$ P. Nilsson, ${ }^{29}$ H. R. Nindhito, ${ }^{52}$ Y. Ninomiya, ${ }^{79}$ A. Nisati, ${ }^{70}$ N. Nishu, ${ }^{58 c}$ R. Nisius, ${ }^{113}$ I. Nitsche, ${ }^{45}$ T. Nitta, ${ }^{176}$ T. Nobe ${ }^{160}$ Y. Noguchi, ${ }^{83}$ M. Nomachi, ${ }^{130}$ I. Nomidis, ${ }^{133}$ M. A. Nomura, ${ }^{29}$ T. Nooney, ${ }^{90}$ M. Nordberg, ${ }^{35}$ N. Norjoharuddeen, ${ }^{132}$ T. Novak ${ }^{89}$ O. Novgorodova, ${ }^{46}$ R. Novotny, ${ }^{139}$ L. Nozka, ${ }^{127}$ K. Ntekas, ${ }^{168}$ E. Nurse,${ }^{92}$ F. Nuti, ${ }^{102}$ F. G. Oakham, ${ }^{33, f}$ H. Oberlack, ${ }^{113}$ J. Ocariz, ${ }^{133}$ A. Ochi, ${ }^{80}$ I. Ochoa ${ }^{38}$ J. P. Ochoa-Ricoux, ${ }^{144 a}$ K. O'Connor, ${ }^{26}$ S. Oda, ${ }^{85}$ S. Odaka, ${ }^{79}$ S. Oerdek, ${ }^{51}$ A. Oh, ${ }^{98}$ S. H. Oh, ${ }^{47}$ C. C. Ohm,${ }^{151}$ H. Oide ${ }^{53 b, 53 a}$ M. L. Ojeda, ${ }^{164}$ H. Okawa, ${ }^{166}$ Y. Okazaki, ${ }^{83}$ Y. Okumura, ${ }^{160}$ T. Okuyama, ${ }^{79}$ A. Olariu, ${ }^{27 b}$ L. F. Oleiro Seabra, ${ }^{137 a}$ S. A. Olivares Pino, ${ }^{144 a}$ D. Oliveira Damazio, ${ }^{29}$ J. L. Oliver, ${ }^{1}$ M. J. R. Olsson, ${ }^{36}$ A. Olszewski, ${ }^{82}$ J. Olszowska, ${ }^{82}$ D. C. O’Neil, ${ }^{149}$ A. Onofre, ${ }^{137 a, 137 e}$ K. Onogi, ${ }^{115}$ P. U. E. Onyisi, ${ }^{11}$ H. Oppen, ${ }^{131}$ M. J. Oreglia, ${ }^{36}$ G. E. Orellana, ${ }^{86}$ Y. Oren, ${ }^{158}$ D. Orestano, ${ }^{72 a, 72 b}$ E. C. Orgill, ${ }^{98}$ N. Orlando, ${ }^{61 b}$ A. A. O'Rourke, ${ }^{44}$ R. S. Orr, ${ }^{164}$ B. Osculati, ${ }^{53 b, 53 a, a}$ V. O'Shea, ${ }^{55}$ R. Ospanov, ${ }^{58 a}$ G. Otero y Garzon, ${ }^{30}$ H. Otono, ${ }^{85}$ M. Ouchrif, ${ }^{34 \mathrm{~d}}$ F. Ould-Saada, ${ }^{131}$ A. Ouraou, ${ }^{142}$ Q. Ouyang, ${ }^{15 a}$ M. Owen, ${ }^{55}$ R. E. Owen, ${ }^{21}$ V. E. Ozcan, ${ }^{12 \mathrm{c}}$ N. Ozturk, ${ }^{8}$ J. Pacalt, ${ }^{127}$ H. A. Pacey, ${ }^{31}$ K. Pachal,${ }^{149}$ A. Pacheco Pages, ${ }^{14}$ L. Pacheco Rodriguez, ${ }^{142}$ C. Padilla Aranda, ${ }^{14}$ S. Pagan Griso, ${ }^{18}$ M. Paganini, ${ }^{180}$ G. Palacino, ${ }^{63}$ S. Palazzo, ${ }^{48}$ S. Palestini,${ }^{35}$ M. Palka, ${ }^{81 b}$ D. Pallin, ${ }^{37}$ I. Panagoulias, ${ }^{10}$ C. E. Pandini, ${ }^{35}$ J. G. Panduro Vazquez, ${ }^{91}$ P. Pani,${ }^{35}$ G. Panizzo, ${ }^{64 a, 64 c}$ L. Paolozzi,${ }^{52}$ T. D. Papadopoulou, ${ }^{10}$ K. Papageorgiou, ${ }^{9, v}$ A. Paramonov, ${ }^{6}$ D. Paredes Hernandez, ${ }^{61 b}$ S. R. Paredes Saenz, ${ }^{132}$ B. Parida, ${ }^{163}$ T. H. Park ${ }^{33}$ A. J. Parker, ${ }^{87}$ K. A. Parker, ${ }^{44}$ M. A. Parker, ${ }^{31}$ F. Parodi, ${ }^{53 b, 53 a}$ J. A. Parsons, ${ }^{38}$ U. Parzefall, ${ }^{50}$ V. R. Pascuzzi, ${ }^{164}$ J. M. P. Pasner, ${ }^{143}$ E. Pasqualucci, ${ }^{70 a}$ S. Passaggio,${ }^{53 b}$ F. Pastore, ${ }^{91}$ P. Pasuwan, ${ }^{43 a, 43 b}$ S. Pataraia, ${ }^{97}$ J. R. Pater, ${ }^{98}$ A. Pathak, ${ }^{178, \mathrm{~g}}$ T. Pauly, ${ }^{35}$ B. Pearson, ${ }^{113}$ M. Pedersen, ${ }^{131}$ L. Pedraza Diaz, ${ }^{117}$ R. Pedro, ${ }^{137 a, 137 b}$ S. V. Peleganchuk, ${ }^{120 b, 120 a}$ O. Penc, ${ }^{138}$ C. Peng, ${ }^{15 \mathrm{~d}}$ H. Peng, ${ }^{58 \mathrm{a}}$ B. S. Peralva ${ }^{78 \mathrm{a}}$ M. M. Perego, ${ }^{129}$ A. P. Pereira Peixoto, ${ }^{137 \mathrm{a}}$ D. V. Perepelitsa, ${ }^{29}$ F. Peri, ${ }^{19}$ L. Perini, ${ }^{66 a, 66 b}$ H. Pernegger, ${ }^{35}$ S. Perrella, ${ }^{67 a, 67 b}$ V. D. Peshekhonov ${ }^{77, a}$ K. Peters, ${ }^{44}$ R. F. Y. Peters, ${ }^{98}$ B. A. Petersen, ${ }^{35}$ T. C. Petersen, ${ }^{39}$ E. Petit,${ }^{56}$ A. Petridis, ${ }^{1}$ C. Petridou, ${ }^{159}$ P. Petroff, ${ }^{129}$ M. Petrov, ${ }^{132}$ F. Petrucci,${ }^{72 a, 72 b}$ M. Pettee,${ }^{180}$ N. E. Pettersson, ${ }^{100}$ A. Peyaud, ${ }^{142}$ R. Pezoa, ${ }^{144 b}$ T. Pham, ${ }^{102}$ F. H. Phillips,${ }^{104}$ P. W. Phillips, ${ }^{141}$ M. W. Phipps, ${ }^{170}$ G. Piacquadio, ${ }^{152}$ E. Pianori, ${ }^{18}$ A. Picazio, ${ }^{100}$ R. H. Pickles, ${ }^{98}$ R. Piegaia, ${ }^{30}$ J. E. Pilcher, ${ }^{36}$ A. D. Pilkington, ${ }^{98}$ M. Pinamonti, ${ }^{71 a, 71 b}$ J. L. Pinfold, ${ }^{3}$ M. Pitt, ${ }^{177}$ L. Pizzimento, ${ }^{71 a, 71 b}$ M.-A. Pleier, ${ }^{29}$ V. Pleskot, ${ }^{140}$ E. Plotnikova, ${ }^{77}$ D. Pluth, ${ }^{76}$ P. Podberezko, ${ }^{120 b, 120 a}$ R. Poettgen, ${ }^{94}$ R. Poggi,${ }^{52}$ L. Poggioli, ${ }^{129}$ I. Pogrebnyak, ${ }^{104}$ D. Pohl,${ }^{24}$ I. Pokharel,${ }^{51}$ G. Polesello, ${ }^{68 a}$ A. Poley, ${ }^{18}$ A. Policicchio, ${ }^{70 a, 70 b}$ R. Polifka, ${ }^{35}$ A. Polini, ${ }^{23 b}$ C. S. Pollard, ${ }^{44}$ V. Polychronakos, ${ }^{29}$ D. Ponomarenko, ${ }^{110}$ L. Pontecorvo, ${ }^{35}$ G. A. Popeneciu, ${ }^{27 \mathrm{~d}}$ D. M. Portillo Quintero, ${ }^{133}$ S. Pospisil,${ }^{139}$ K. Potamianos, ${ }^{44}$ I. N. Potrap,${ }^{77}$ C. J. Potter, ${ }^{31}$ H. Potti, ${ }^{11}$ T. Poulsen, ${ }^{94}$ J. Poveda, ${ }^{35}$ T. D. Powell, ${ }^{146}$ M. E. Pozo Astigarraga, ${ }^{35}$ P. Pralavorio, ${ }^{99}$ S. Prell, ${ }^{76}$ D. Price, ${ }^{98}$ M. Primavera, ${ }^{65 a}$ S. Prince, ${ }^{101}$ M. L. Proffitt, ${ }^{145}$ N. Proklova, ${ }^{110}$ K. Prokofiev, ${ }^{61 \mathrm{c}}$ F. Prokoshin, ${ }^{144 \mathrm{~b}}$ S. Protopopescu, ${ }^{29}$ J. Proudfoot, ${ }^{6}$ M. Przybycien, ${ }^{81 a}$ A. Puri, ${ }^{170}$ P. Puzo, ${ }^{129}$ J. Qian, ${ }^{103}$ Y. Qin, ${ }^{98}$ A. Quadt,${ }^{51}$ M. Queitsch-Maitland, ${ }^{44}$ A. Qureshi, ${ }^{1}$ P. Rados, ${ }^{102}$ F. Ragusa, ${ }^{66 a, 66 b}$ G. Rahal, ${ }^{95}$ J. A. Raine, ${ }^{52}$ S. Rajagopalan, ${ }^{29}$ A. Ramirez Morales, ${ }^{90}$ K. Ran, ${ }^{15 a}$ T. Rashid, ${ }^{129}$ S. Raspopov, ${ }^{5}$ M. G. Ratti, ${ }^{66 a, 66 b}$ D. M. Rauch,${ }^{44}$ F. Rauscher, ${ }^{112}$ S. Rave, ${ }^{97}$ B. Ravina, ${ }^{146}$ I. Ravinovich, ${ }^{177}$ J. H. Rawling, ${ }^{98}$ M. Raymond, ${ }^{35}$ A. L. Read, ${ }^{131}$ N. P. Readioff, ${ }^{56}$ M. Reale, ${ }^{65 a, 65 b}$ D. M. Rebuzzi, ${ }^{68,68 b}$ A. Redelbach, ${ }^{174}$ G. Redlinger ${ }^{29}$ R. Reece, ${ }^{143}$ R. G. Reed ${ }^{32 c}$ K. Reeves,${ }^{42}$ L. Rehnisch,${ }^{19}$ J. Reichert, ${ }^{134}$ D. Reikher, ${ }^{158}$ A. Reiss,${ }^{97}$ A. Rej, ${ }^{148}$ C. Rembser, ${ }^{35}$ H. Ren, ${ }^{15 d}$ M. Rescigno, ${ }^{70 a}$ S. Resconi, ${ }^{66 a}$ E. D. Resseguie, ${ }^{134}$ S. Rettie, ${ }^{172}$ E. Reynolds, ${ }^{21}$ O. L. Rezanova, ${ }^{120 b, 120 \mathrm{a}}$ P. Reznicek, ${ }^{140}$ E. Ricci, ${ }^{73 a, 73 b}$ R. Richter, ${ }^{113}$ 
S. Richter, ${ }^{44}$ E. Richter-Was, ${ }^{81 b}$ O. Ricken, ${ }^{24}$ M. Ridel, ${ }^{133}$ P. Rieck, ${ }^{113}$ C. J. Riegel, ${ }^{179}$ O. Rifki, ${ }^{44}$ M. Rijssenbeek, ${ }^{152}$ A. Rimoldi, ${ }^{68 a, 68 b}$ M. Rimoldi, ${ }^{20}$ L. Rinaldi, ${ }^{23 b}$ G. Ripellino, ${ }^{151}$ B. Ristic,${ }^{87}$ E. Ritsch, ${ }^{35}$ I. Riu, ${ }^{14}$ J. C. Rivera Vergara, ${ }^{144 a}$ F. Rizatdinova, ${ }^{126}$ E. Rizvi, ${ }^{90}$ C. Rizzi, ${ }^{14}$ R. T. Roberts, ${ }^{98}$ S. H. Robertson, ${ }^{101,0}$ D. Robinson, ${ }^{31}$ J. E. M. Robinson, ${ }^{44}$ A. Robson, ${ }^{55}$ E. Rocco,${ }^{97}$ C. Roda, ${ }^{69 a, 69 b}$ Y. Rodina, ${ }^{99}$ S. Rodriguez Bosca,${ }^{171}$ A. Rodriguez Perez, ${ }^{14}$

D. Rodriguez Rodriguez, ${ }^{171}$ A. M. Rodríguez Vera, ${ }^{165 b}$ S. Roe,${ }^{35}$ C. S. Rogan,${ }^{57}$ O. Røhne, ${ }^{131}$ R. Röhrig, ${ }^{113}$ C. P. A. Roland, ${ }^{63}$ J. Roloff, ${ }^{57}$ A. Romaniouk, ${ }^{110}$ M. Romano, ${ }^{23 b, 23 a}$ N. Rompotis, ${ }^{88}$ M. Ronzani, ${ }^{122}$ L. Roos, ${ }^{133}$ S. Rosati, ${ }^{70 a}$ K. Rosbach ${ }^{50}$ N-A. Rosien, ${ }^{51}$ B. J. Rosser, ${ }^{134}$ E. Rossi, ${ }^{44}$ E. Rossi, ${ }^{72 a, 72 b}$ E. Rossi, ${ }^{67 a, 67 b}$ L. P. Rossi, ${ }^{53 b}$ L. Rossini, ${ }^{66 a, 66 b}$ J. H. N. Rosten, ${ }^{31}$ R. Rosten, ${ }^{14}$ M. Rotaru, ${ }^{27 b}$ J. Rothberg, ${ }^{145}$ D. Rousseau, ${ }^{129}$ D. Roy, ${ }^{32 \mathrm{c}}$ A. Rozanov, ${ }^{99}$ Y. Rozen, ${ }^{157}$ X. Ruan,${ }^{32 \mathrm{c}}$ F. Rubbo, ${ }^{150}$ F. Rühr, ${ }^{50}$ A. Ruiz-Martinez, ${ }^{171}$ Z. Rurikova,${ }^{50}$ N. A. Rusakovich, ${ }^{77}$ H. L. Russell, ${ }^{101}$ J. P. Rutherfoord, ${ }^{7}$ E. M. Rüttinger, ${ }^{44, \text { rr }}$ Y. F. Ryabov, ${ }^{135}$ M. Rybar, ${ }^{38}$ G. Rybkin, ${ }^{129}$ S. Ryu, ${ }^{6}$ A. Ryzhov, ${ }^{121}$ G. F. Rzehorz, ${ }^{51}$ P. Sabatini, ${ }^{51}$ G. Sabato, ${ }^{118}$ S. Sacerdoti, ${ }^{129}$ H. F-W. Sadrozinski, ${ }^{143}$ R. Sadykov, ${ }^{77}$ F. Safai Tehrani, ${ }^{70 a}$ P. Saha, ${ }^{119}$ M. Sahinsoy, ${ }^{59 a}$ A. Sahu, ${ }^{179}$ M. Saimpert, ${ }^{44}$ M. Saito, ${ }^{160}$ T. Saito, ${ }^{160}$ H. Sakamoto, ${ }^{160}$ A. Sakharov, ${ }^{122, i i}$ D. Salamani,${ }^{52}$ G. Salamanna, ${ }^{72 a, 72 b}$ J. E. Salazar Loyola, ${ }^{144 b}$ P. H. Sales De Bruin, ${ }^{169}$ D. Salihagic, ${ }^{113, a}$ A. Salnikov, ${ }^{150}$ J. Salt, ${ }^{171}$ D. Salvatore, ${ }^{40 \mathrm{~b}, 40 \mathrm{a}}$ F. Salvatore, ${ }^{153}$ A. Salvucci, ${ }^{61 \mathrm{a}, 61 \mathrm{~b}, 61 \mathrm{c}}$ A. Salzburger, ${ }^{35}$ J. Samarati, ${ }^{35}$ D. Sammel, ${ }^{50}$ D. Sampsonidis, ${ }^{159}$ D. Sampsonidou, ${ }^{159}$ J. Sánchez, ${ }^{171}$ A. Sanchez Pineda, ${ }^{64 a, 64 c}$ H. Sandaker, ${ }^{131}$ C. O. Sander, ${ }^{44}$ M. Sandhoff, ${ }^{179}$ C. Sandoval, ${ }^{22}$ D. P. C. Sankey, ${ }^{141}$ M. Sannino, ${ }^{53 b, 53 a}$ Y. Sano, ${ }^{115}$ A. Sansoni, ${ }^{49}$ C. Santoni,${ }^{37}$ H. Santos, ${ }^{137 a}$ I. Santoyo Castillo, ${ }^{153}$ A. Santra, ${ }^{171}$ A. Sapronov, ${ }^{77}$ J. G. Saraiva, ${ }^{137 a, 137 d}$ O. Sasaki, ${ }^{79}$ K. Sato, ${ }^{166}$ E. Sauvan, ${ }^{5}$ P. Savard,${ }^{164, f}$ N. Savic, ${ }^{113}$ R. Sawada, ${ }^{160}$ C. Sawyer, ${ }^{141}$ L. Sawyer, ${ }^{93, x}$ C. Sbarra, ${ }^{23 b}$ A. Sbrizzi, ${ }^{23 a}$ T. Scanlon, ${ }^{92}$ J. Schaarschmidt, ${ }^{145}$ P. Schacht, ${ }^{113}$ B. M. Schachtner, ${ }^{112}$ D. Schaefer, ${ }^{36}$ L. Schaefer, ${ }^{134}$ J. Schaeffer,${ }^{97}$ S. Schaepe,${ }^{35}$ U. Schäfer, ${ }^{97}$ A. C. Schaffer, ${ }^{129}$ D. Schaile, ${ }^{112}$ R. D. Schamberger, ${ }^{152}$ N. Scharmberg, ${ }^{98}$ V. A. Schegelsky, ${ }^{135}$ D. Scheirich, ${ }^{140}$ F. Schenck, ${ }^{19}$ M. Schernau, ${ }^{168}$ C. Schiavi, ${ }^{53 b, 53 a}$ S. Schier, ${ }^{143}$ L. K. Schildgen, ${ }^{24}$ Z. M. Schillaci, ${ }^{26}$ E. J. Schioppa, ${ }^{35}$ M. Schioppa ${ }^{40 b, 40 a}$ K. E. Schleicher, ${ }^{50}$

S. Schlenker, ${ }^{35}$ K. R. Schmidt-Sommerfeld, ${ }^{113}$ K. Schmieden, ${ }^{35}$ C. Schmitt,,${ }^{97}$ S. Schmitt, ${ }^{44}$ S. Schmitz, ${ }^{97}$

J. C. Schmoeckel, ${ }^{44}$ U. Schnoor, ${ }^{50}$ L. Schoeffel, ${ }^{142}$ A. Schoening, ${ }^{59 b}$ E. Schopf, ${ }^{132}$ M. Schott, ${ }^{97}$ J. F. P. Schouwenberg, ${ }^{117}$ J. Schovancova, ${ }^{35}$ S. Schramm, ${ }^{52}$ A. Schulte, ${ }^{97}$ H-C. Schultz-Coulon, ${ }^{59 a}$ M. Schumacher, ${ }^{50}$ B. A. Schumm, ${ }^{143}$ Ph. Schune, ${ }^{142}$ A. Schwartzman, ${ }^{150}$ T. A. Schwarz, ${ }^{103}$ Ph. Schwemling, ${ }^{142}$ R. Schwienhorst, ${ }^{104}$ A. Sciandra, ${ }^{24}$ G. Sciolla, ${ }^{26}$ M. Scornajenghi, ${ }^{40 b, 40 a}$ F. Scuri, ${ }^{69 a}$ F. Scutti, ${ }^{102}$ L. M. Scyboz, ${ }^{113}$ C. D. Sebastiani, ${ }^{70 a}, 70 b$ P. Seema, ${ }^{19}$ S. C. Seidel, ${ }^{116}$ A. Seiden, ${ }^{143}$ T. Seiss, ${ }^{36}$ J. M. Seixas, ${ }^{78 b}$ G. Sekhniaidze, ${ }^{67 a}$ K. Sekhon, ${ }^{103}$ S. J. Sekula, ${ }^{41}$ N. Semprini-Cesari, ${ }^{23 b, 23 a}$ S. Sen, ${ }^{47}$ S. Senkin, ${ }^{37}$ C. Serfon, ${ }^{131}$ L. Serin,${ }^{129}$ L. Serkin, ${ }^{64 a, 64 b}$ M. Sessa ${ }^{58 a}$ H. Severini, ${ }^{125}$ F. Sforza ${ }^{167}$ A. Sfyrla, ${ }^{52}$ E. Shabalina, ${ }^{51}$ J. D. Shahinian, ${ }^{143}$ N. W. Shaikh, ${ }^{43,43 b}$ D. Shaked Renous, ${ }^{177}$ L. Y. Shan, ${ }^{15 a}$ R. Shang, ${ }^{170}$ J. T. Shank, ${ }^{25}$ M. Shapiro, ${ }^{18}$ A. S. Sharma, ${ }^{1}$ A. Sharma, ${ }^{132}$ P. B. Shatalov, ${ }^{109}$ K. Shaw, ${ }^{153}$ S. M. Shaw, ${ }^{98}$ A. Shcherbakova, ${ }^{135}$ Y. Shen, ${ }^{125}$ N. Sherafati, ${ }^{33}$ A. D. Sherman ${ }^{25}$ P. Sherwood, ${ }^{92}$ L. Shi, ${ }^{155, s s}$ S. Shimizu, ${ }^{79}$ C. O. Shimmin ${ }^{180}$ Y. Shimogama, ${ }^{176}$ M. Shimojima, ${ }^{114}$

I. P. J. Shipsey, ${ }^{132}$ S. Shirabe, ${ }^{85}$ M. Shiyakova ${ }^{77}$ J. Shlomi, ${ }^{177}$ A. Shmeleva ${ }^{108}$ D. Shoaleh Saadi, ${ }^{107}$ M. J. Shochet, ${ }^{36}$

S. Shojaii, ${ }^{102}$ D. R. Shope, ${ }^{125}$ S. Shrestha, ${ }^{123}$ E. Shulga, ${ }^{110}$ P. Sicho, ${ }^{138}$ A. M. Sickles, ${ }^{170}$ P. E. Sidebo, ${ }^{151}$

E. Sideras Haddad, ${ }^{32 c}$ O. Sidiropoulou, ${ }^{35}$ A. Sidoti, ${ }^{23 b, 23 a}$ F. Siegert, ${ }^{46}$ Dj. Sijacki, ${ }^{16}$ J. Silva, ${ }^{137 a}$ M. Silva Jr., ${ }^{178}$ M. V. Silva Oliveira, ${ }^{78 a}$ S. B. Silverstein, ${ }^{43 a}$ S. Simion, ${ }^{129}$ E. Simioni, ${ }^{97}$ M. Simon, ${ }^{97}$ R. Simoniello, ${ }^{97}$ P. Sinervo, ${ }^{164}$ N. B. Sinev, ${ }^{128}$ M. Sioli, ${ }^{23 b, 23 a}$ I. Siral, ${ }^{103}$ S. Yu. Sivoklokov, ${ }^{111}$ J. Sjölin, ${ }^{43 a, 43 b}$ P. Skubic, ${ }^{125}$ M. Slater, ${ }^{21}$ T. Slavicek, ${ }^{139}$ M. Slawinska, ${ }^{82}$ K. Sliwa, ${ }^{167}$ R. Slovak, ${ }^{140}$ V. Smakhtin, ${ }^{177}$ B. H. Smart, ${ }^{5}$ J. Smiesko, ${ }^{28 a}$ N. Smirnov, ${ }^{110}$ S. Yu. Smirnov, ${ }^{110}$ Y. Smirnov, ${ }^{110}$ L. N. Smirnova, ${ }^{111}$ O. Smirnova, ${ }^{94}$ J. W. Smith, ${ }^{51}$ M. Smizanska, ${ }^{87}$ K. Smolek, ${ }^{139}$ A. Smykiewicz, ${ }^{82}$ A. A. Snesarev, ${ }^{108}$ I. M. Snyder, ${ }^{128}$ S. Snyder, ${ }^{29}$ R. Sobie,${ }^{173,0}$ A. M. Soffa ${ }^{168}$ A. Soffer, ${ }^{158}$ A. Søgaard, ${ }^{48}$ F. Sohns, ${ }^{51}$ G. Sokhrannyi, ${ }^{89}$ C. A. Solans Sanchez, ${ }^{35}$ M. Solar, ${ }^{139}$ E. Yu. Soldatov, ${ }^{110}$ U. Soldevila, ${ }^{171}$ A. A. Solodkov, ${ }^{121}$ A. Soloshenko, ${ }^{77}$ O. V. Solovyanov, ${ }^{121}$ V. Solovyev, ${ }^{135}$ P. Sommer, ${ }^{146}$ H. Son, ${ }^{167}$ W. Song, ${ }^{141}$ W. Y. Song,${ }^{165 b}$ A. Sopczak, ${ }^{139}$ F. Sopkova, ${ }^{28 b}$ C. L. Sotiropoulou, ${ }^{69 a, 69 b}$ S. Sottocornola, ${ }^{68 a, 68 b}$ R. Soualah,${ }^{64 a, 64 c, t t}$ A. M. Soukharev, ${ }^{120 b, 120 a}$ D. South, ${ }^{44}$ S. Spagnolo, ${ }^{65 a, 65 b}$ M. Spalla, ${ }^{113}$ M. Spangenberg, ${ }^{175}$ F. Spanò,${ }^{91}$ D. Sperlich, ${ }^{19}$ T. M. Spieker, ${ }^{59 a}$ R. Spighi, ${ }^{23 b}$ G. Spigo, ${ }^{35}$ L. A. Spiller, ${ }^{102}$ D. P. Spiteri, ${ }^{55}$ M. Spousta, ${ }^{140}$ A. Stabile,${ }^{66 a, 66 b}$ R. Stamen, ${ }^{59 a}$ S. Stamm, ${ }^{19}$ E. Stanecka,${ }^{82}$ R. W. Stanek, ${ }^{6}$ C. Stanescu, ${ }^{72 a}$ B. Stanislaus, ${ }^{132}$ M. M. Stanitzki, ${ }^{44}$ B. Stapf, ${ }^{118}$ E. A. Starchenko, ${ }^{121}$ G. H. Stark, ${ }^{143}$ J. Stark, ${ }^{56}$ S. H Stark,${ }^{39}$ P. Staroba, ${ }^{138}$ P. Starovoitov, ${ }^{59 a}$ S. Stärz, ${ }^{101}$ R. Staszewski, ${ }^{82}$ M. Stegler ${ }^{44}$ P. Steinberg, ${ }^{29}$ B. Stelzer, ${ }^{149}$ H. J. Stelzer, ${ }^{35}$ O. Stelzer-Chilton, ${ }^{165 a}$ H. Stenzel, ${ }^{54}$ T. J. Stevenson, ${ }^{153}$ G. A. Stewart, ${ }^{35}$ M. C. Stockton, ${ }^{35}$ G. Stoicea, ${ }^{27 b}$ P. Stolte ${ }^{51}$ S. Stonjek ${ }^{113}$ A. Straessner, ${ }^{46}$ J. Strandberg, ${ }^{151}$ S. Strandberg, ${ }^{43 a, 43 b}$ M. Strauss, ${ }^{125}$ P. Strizenec, ${ }^{28 b}$ R. Ströhmer, ${ }^{174}$ D. M. Strom, ${ }^{128}$ R. Stroynowski, ${ }^{41}$ A. Strubig, ${ }^{48}$ S. A. Stucci, ${ }^{29}$ B. Stugu, ${ }^{17}$ J. Stupak, ${ }^{125}$ N. A. Styles, ${ }^{44}$ D. Su, ${ }^{150}$ J. Su, ${ }^{136}$ 
S. Suchek, ${ }^{59 a}$ Y. Sugaya, ${ }^{130}$ M. Suk, ${ }^{139}$ V. V. Sulin, ${ }^{108}$ M. J. Sullivan,${ }^{88}$ D. M. S. Sultan, ${ }^{52}$ S. Sultansoy,${ }^{4 c}$ T. Sumida, ${ }^{83}$ S. Sun, ${ }^{103}$ X. Sun, ${ }^{3}$ K. Suruliz, ${ }^{153}$ C. J. E. Suster, ${ }^{154}$ M. R. Sutton, ${ }^{153}$ S. Suzuki, ${ }^{79}$ M. Svatos, ${ }^{138}$ M. Swiatlowski, ${ }^{36}$

S. P. Swift, ${ }^{2}$ A. Sydorenko, ${ }^{97}$ I. Sykora, ${ }^{28 a}$ M. Sykora, ${ }^{140}$ T. Sykora, ${ }^{140}$ D. Ta,${ }^{97}$ K. Tackmann, ${ }^{44, u u}$ J. Taenzer, ${ }^{158}$ A. Taffard, ${ }^{168}$ R. Tafirout, ${ }^{165 a}$ E. Tahirovic, ${ }^{90}$ N. Taiblum, ${ }^{158}$ H. Takai, ${ }^{29}$ R. Takashima, ${ }^{84}$ E. H. Takasugi, ${ }^{113}$ K. Takeda, ${ }^{80}$ T. Takeshita, ${ }^{147}$ Y. Takubo, ${ }^{79}$ M. Talby, ${ }^{99}$ A. A. Talyshev, ${ }^{120 b, 120 a}$ J. Tanaka, ${ }^{160}$ M. Tanaka, ${ }^{162}$ R. Tanaka, ${ }^{129}$

B. B. Tannenwald, ${ }^{123}$ S. Tapia Araya, ${ }^{170}$ S. Tapprogge, ${ }^{97}$ A. Tarek Abouelfadl Mohamed, ${ }^{133}$ S. Tarem, ${ }^{157}$ G. Tarna,${ }^{27 b, r}$ G. F. Tartarelli, ${ }^{66 \mathrm{a}} \mathrm{P}$. Tas, ${ }^{140}$ M. Tasevsky, ${ }^{138}$ T. Tashiro, ${ }^{83}$ E. Tassi ${ }^{40 \mathrm{~b}, 40 \mathrm{a}}$ A. Tavares Delgado, ${ }^{137 \mathrm{a}, 137 \mathrm{~b}}$ Y. Tayalati, ${ }^{34 \mathrm{e}}$ A. J. Taylor, ${ }^{48}$ G. N. Taylor, ${ }^{102}$ P. T. E. Taylor, ${ }^{102}$ W. Taylor, ${ }^{165 b}$ A. S. Tee, ${ }^{87}$ R. Teixeira De Lima, ${ }^{150}$ P. Teixeira-Dias, ${ }^{91}$ H. Ten Kate, ${ }^{35}$ J. J. Teoh, ${ }^{118}$ S. Terada,${ }^{79}$ K. Terashi, ${ }^{160}$ J. Terron, ${ }^{96}$ S. Terzo, ${ }^{14}$ M. Testa, ${ }^{49}$ R. J. Teuscher, ${ }^{164,0}$ S. J. Thais, ${ }^{180}$

T. Theveneaux-Pelzer, ${ }^{44}$ F. Thiele, ${ }^{39}$ D. W. Thomas, ${ }^{91}$ J. P. Thomas, ${ }^{21}$ A. S. Thompson, ${ }^{55}$ P. D. Thompson, ${ }^{21}$

L. A. Thomsen, ${ }^{180}$ E. Thomson, ${ }^{134}$ Y. Tian, ${ }^{38}$ R. E. Ticse Torres, ${ }^{51}$ V. O. Tikhomirov, ${ }^{108, v v}$ Yu. A. Tikhonov, ${ }^{120 b, 120 a}$

S. Timoshenko, ${ }^{110}$ P. Tipton, ${ }^{180}$ S. Tisserant, ${ }^{99} \mathrm{~K}$. Todome, ${ }^{162}$ S. Todorova-Nova, ${ }^{5}$ S. Todt,${ }^{46}$ J. Tojo, ${ }^{85}$ S. Tokár, ${ }^{28 a}$ K. Tokushuku, ${ }^{79}$ E. Tolley ${ }^{123}$ K. G. Tomiwa, ${ }^{32 c}$ M. Tomoto,${ }^{115}$ L. Tompkins, ${ }^{150, \text { hh }}$ K. Toms, ${ }^{116}$ B. Tong,${ }^{57}$ P. Tornambe, ${ }^{50}$ E. Torrence, ${ }^{128}$ H. Torres ${ }^{46}$ E. Torró Pastor, ${ }^{145}$ C. Tosciri, ${ }^{132}$ J. Toth, ${ }^{99, w w}$ F. Touchard, ${ }^{99}$ D. R. Tovey, ${ }^{146}$ C. J. Treado, ${ }^{122}$ T. Trefzger,${ }^{174}$ F. Tresoldi, ${ }^{153}$ A. Tricoli, ${ }^{29}$ I. M. Trigger, ${ }^{165 a}$ S. Trincaz-Duvoid, ${ }^{133}$ W. Trischuk, ${ }^{164}$ B. Trocmé ${ }^{56}$ A. Trofymov, ${ }^{129}$ C. Troncon, ${ }^{66 a}$ M. Trovatelli, ${ }^{173}$ F. Trovato, ${ }^{153}$ L. Truong, ${ }^{32 b}$ M. Trzebinski ${ }^{82}$ A. Trzupek, ${ }^{82}$ F. Tsai, ${ }^{44}$ J. C-L. Tseng, ${ }^{132}$ P. V. Tsiareshka, ${ }^{105, j \mathrm{jj}}$ A. Tsirigotis, ${ }^{159}$ N. Tsirintanis, ${ }^{9}$ V. Tsiskaridze,${ }^{152}$ E. G. Tskhadadze, ${ }^{156 a}$ I. I. Tsukerman, ${ }^{109}$ V. Tsulaia, ${ }^{18}$ S. Tsuno, ${ }^{79}$ D. Tsybychev, ${ }^{152,163} \mathrm{Y}$. Tu ${ }^{61 \mathrm{~b}}$ A. Tudorache, ${ }^{27 \mathrm{~b}} \mathrm{~V}$. Tudorache,${ }^{27 \mathrm{~b}}$ T. T. Tulbure, ${ }^{27 a}$ A. N. Tuna, ${ }^{57}$ S. Turchikhin, ${ }^{77}$ D. Turgeman, ${ }^{177}$ I. Turk Cakir, ${ }^{4 b, x x}$ R. J. Turner, ${ }^{21}$ R. T. Turra, ${ }^{66 a}$ P. M. Tuts,${ }^{38}$ S. Tzamarias, ${ }^{159}$ E. Tzovara, ${ }^{97}$ G. Ucchielli, ${ }^{45}$ I. Ueda, ${ }^{79}$ M. Ughetto, ${ }^{43 a, 43 b}$ F. Ukegawa, ${ }^{166}$ G. Unal, ${ }^{35}$ A. Undrus, ${ }^{29}$ G. Unel, ${ }^{168}$ F. C. Ungaro, ${ }^{102}$ Y. Unno, ${ }^{79}$ K. Uno, ${ }^{160}$ J. Urban, ${ }^{28 b}$ P. Urquijo, ${ }^{102}$ G. Usai, ${ }^{8}$ J. Usui, ${ }^{79}$ L. Vacavant, ${ }^{99}$ V. Vacek, ${ }^{139}$ B. Vachon, ${ }^{101}$ K. O. H. Vadla, ${ }^{131}$ A. Vaidya,${ }^{92}$ C. Valderanis, ${ }^{112}$ E. Valdes Santurio,${ }^{43 a, 43 b}$ M. Valente, ${ }^{52}$ S. Valentinetti, ${ }^{23 b, 23 a}$ A. Valero, ${ }^{171}$ L. Valéry, ${ }^{44}$ R. A. Vallance, ${ }^{21}$ A. Vallier, ${ }^{5}$ J. A. Valls Ferrer,${ }^{171}$ T. R. Van Daalen, ${ }^{14}$ H. Van der Graaf, ${ }^{118}$ P. Van Gemmeren, ${ }^{6}$ I. Van Vulpen, ${ }^{118}$ M. Vanadia,${ }^{71 a, 71 b}$ W. Vandelli, ${ }^{35}$ A. Vaniachine,${ }^{163}$ P. Vankov, ${ }^{118}$ R. Vari, ${ }^{70 a}$

E. W. Varnes, ${ }^{7}$ C. Varni, ${ }^{53 \mathrm{~b}, 53 \mathrm{a}}$ T. Varol, ${ }^{41}$ D. Varouchas, ${ }^{129}$ K. E. Varvell, ${ }^{154}$ G. A. Vasquez, ${ }^{144 \mathrm{~b}}$ J. G. Vasquez, ${ }^{180}$

F. Vazeille, ${ }^{37}$ D. Vazquez Furelos,${ }^{14}$ T. Vazquez Schroeder, ${ }^{35}$ J. Veatch,${ }^{51}$ V. Vecchio, ${ }^{72 a, 72 b}$ L. M. Veloce, ${ }^{164}$ F. Veloso, ${ }^{137 a, 137 c}$ S. Veneziano, ${ }^{70 \mathrm{a}}$ A. Ventura, ${ }^{65 a, 65 \mathrm{~b}} \mathrm{~N}$. Venturi, ${ }^{35} \mathrm{~V}$. Vercesi, ${ }^{68 \mathrm{a}} \mathrm{M}$. Verducci, ${ }^{72 \mathrm{a}, 72 \mathrm{~b}} \mathrm{C} . \mathrm{M}$. Vergel Infante, ${ }^{76} \mathrm{C}$. Vergis, ${ }^{24}$ W. Verkerke, ${ }^{118}$ A. T. Vermeulen, ${ }^{118}$ J. C. Vermeulen, ${ }^{118}$ M. C. Vetterli, ${ }^{149, \mathrm{f}}$ N. Viaux Maira, ${ }^{144 \mathrm{~b}}$ M. Vicente Barreto Pinto, ${ }^{52}$ I. Vichou, ${ }^{170, a}$ T. Vickey, ${ }^{146}$ O. E. Vickey Boeriu, ${ }^{146}$ G. H. A. Viehhauser, ${ }^{132}$ S. Viel, ${ }^{18}$ L. Vigani, ${ }^{132}$ M. Villa, ${ }^{23 b, 23 a}$ M. Villaplana Perez, ${ }^{66,66 \mathrm{~b}}$ E. Vilucchi, ${ }^{49}$ M. G. Vincter, ${ }^{33}$ V. B. Vinogradov, ${ }^{77}$ A. Vishwakarma, ${ }^{44}$ C. Vittori, ${ }^{23 b, 23 a}$ I. Vivarelli, ${ }^{153}$ S. Vlachos,${ }^{10}$ M. Vogel, ${ }^{179}$ P. Vokac, ${ }^{139}$ G. Volpi, ${ }^{14}$ S. E. von Buddenbrock,${ }^{32 c}$ E. Von Toerne,${ }^{24}$ V. Vorobel, ${ }^{140}$ K. Vorobev, ${ }^{110}$ M. Vos, ${ }^{171}$ J. H. Vossebeld ${ }^{88}$ N. Vranjes ${ }^{16}$ M. Vranjes Milosavljevic, ${ }^{16}$ V. Vrba ${ }^{139}$ M. Vreeswijk, ${ }^{118}$ T. Šfiligoj, ${ }^{89}$ R. Vuillermet, ${ }^{35}$ I. Vukotic, ${ }^{36}$ T. Ženiš ${ }^{28 a}$ L. Živković, ${ }^{16}$ P. Wagner, ${ }^{24}$ W. Wagner, ${ }^{179}$ J. Wagner-Kuhr, ${ }^{112}$ H. Wahlberg, ${ }^{86}$ S. Wahrmund, ${ }^{46}$ K. Wakamiya ${ }^{80}$ V. M. Walbrecht, ${ }^{113}$ J. Walder,${ }^{87}$ R. Walker, ${ }^{12}$ S. D. Walker, ${ }^{91}$ W. Walkowiak, ${ }^{148}$ V. Wallangen, ${ }^{43 a, 43 b}$ A. M. Wang, ${ }^{57}$ C. Wang, ${ }^{58 b}$ F. Wang, ${ }^{178}$ H. Wang, ${ }^{18}$ H. Wang, ${ }^{3}$ J. Wang, ${ }^{154}$ J. Wang, ${ }^{59 \mathrm{~b}}$ P. Wang, ${ }^{41}$ Q. Wang, ${ }^{125}$ R.-J. Wang, ${ }^{133}$ R. Wang, ${ }^{58 \mathrm{a}}$ R. Wang, ${ }^{6}$ S. M. Wang, ${ }^{155}$ W. T. Wang, ${ }^{58 a}$ W. Wang, ${ }^{15 c, y y}$ W. X. Wang, ${ }^{58 a, y y}$ Y. Wang, ${ }^{58 a, 11}$ Z. Wang, ${ }^{58 c}$ C. Wanotayaroj, ${ }^{44}$ A. Warburton, ${ }^{101}$ C. P. Ward, ${ }^{31}$ D. R. Wardrope, ${ }^{92}$ A. Washbrook, ${ }^{48}$ P. M. Watkins, ${ }^{21}$ A. T. Watson, ${ }^{21}$ M. F. Watson, ${ }^{21}$ G. Watts, ${ }^{145}$ S. Watts ${ }^{98}$ B. M. Waugh, ${ }^{92}$ A. F. Webb, ${ }^{11}$ S. Webb,${ }^{97}$ C. Weber, ${ }^{180}$ M. S. Weber, ${ }^{20}$ S. A. Weber, ${ }^{33}$ S. M. Weber, ${ }^{59 a}$ A. R. Weidberg, ${ }^{132}$ J. Weingarten, ${ }^{45}$ M. Weirich, ${ }^{97}$ C. Weiser, ${ }^{50}$ P. S. Wells, ${ }^{35}$ T. Wenaus, ${ }^{29}$ T. Wengler, ${ }^{35}$ S. Wenig, ${ }^{35}$ N. Wermes,${ }^{24}$ M. D. Werner, ${ }^{76}$ P. Werner, ${ }^{35}$ M. Wessels, ${ }^{59 a}$ T. D. Weston, ${ }^{20}$ K. Whalen, ${ }^{128}$ N. L. Whallon, ${ }^{145}$ A. M. Wharton, ${ }^{87}$ A. S. White, ${ }^{103}$ A. White, ${ }^{8}$ M. J. White, ${ }^{1}$ R. White, ${ }^{144 b}$ D. Whiteson, ${ }^{168}$ B. W. Whitmore, ${ }^{87}$ F. J. Wickens, ${ }^{141}$ W. Wiedenmann, ${ }^{178}$ M. Wielers, ${ }^{141}$ C. Wiglesworth, ${ }^{39}$

L. A. M. Wiik-Fuchs, ${ }^{50}$ F. Wilk, ${ }^{98}$ H. G. Wilkens,${ }^{35}$ L. J. Wilkins, ${ }^{91}$ H. H. Williams, ${ }^{134}$ S. Williams, ${ }^{31}$ C. Willis, ${ }^{104}$

S. Willocq, ${ }^{100}$ J. A. Wilson, ${ }^{21}$ I. Wingerter-Seez, ${ }^{5}$ E. Winkels, ${ }^{153}$ F. Winklmeier, ${ }^{128}$ O. J. Winston, ${ }^{153}$ B. T. Winter,${ }^{50}$ M. Wittgen, ${ }^{150}$ M. Wobisch, ${ }^{93}$ A. Wolf, ${ }^{97}$ T. M. H. Wolf, ${ }^{118}$ R. Wolff, ${ }^{99}$ J. Wollrath, ${ }^{50}$ M. W. Wolter, ${ }^{82}$ H. Wolters, ${ }^{137 a, 137 c}$ V. W. S. Wong, ${ }^{172}$ N. L. Woods, ${ }^{143}$ S. D. Worm, ${ }^{21}$ B. K. Wosiek, ${ }^{82}$ K. W. Woźniak, ${ }^{82}$ K. Wraight, ${ }^{55}$ M. Wu, ${ }^{36}$ S. L. Wu, ${ }^{178}$ X. Wu, ${ }^{52}$ Y. Wu, ${ }^{58 \mathrm{a}}$ T. R. Wyatt, ${ }^{98}$ B. M. Wynne, ${ }^{48}$ S. Xella, ${ }^{39}$ Z. Xi,${ }^{103}$ L. Xia, ${ }^{175}$ D. Xu, ${ }^{15 a}$ H. Xu, ${ }^{58 a, r}$ L. Xu, ${ }^{29}$ T. Xu, ${ }^{142}$ W. Xu, ${ }^{103}$ Z. Xu, ${ }^{150}$ B. Yabsley, ${ }^{154}$ S. Yacoob,${ }^{32 a}$ K. Yajima, ${ }^{130}$ D. P. Yallup, ${ }^{92}$ D. Yamaguchi, ${ }^{162}$ Y. Yamaguchi, ${ }^{162}$ A. Yamamoto, ${ }^{79}$ T. Yamanaka, ${ }^{160}$ F. Yamane, ${ }^{80}$ M. Yamatani, ${ }^{160}$ T. Yamazaki, ${ }^{160}$ Y. Yamazaki, ${ }^{80}$ Z. Yan, ${ }^{25}$ H. J. Yang, ${ }^{58 c, 58 d}$ 
H. T. Yang, ${ }^{18}$ S. Yang, ${ }^{75}$ Y. Yang, ${ }^{160}$ Z. Yang, ${ }^{17}$ W-M. Yao, ${ }^{18}$ Y. C. Yap, ${ }^{44}$ Y. Yasu, ${ }^{79}$ E. Yatsenko, ${ }^{58,58 d}$ J. Ye, ${ }^{41}$ S. Ye, ${ }^{29}$ I. Yeletskikh, ${ }^{77}$ E. Yigitbasi, ${ }^{25}$ E. Yildirim, ${ }^{97}$ K. Yorita, ${ }^{176}$ K. Yoshihara, ${ }^{134}$ C. J. S. Young, ${ }^{35}$ C. Young, ${ }^{150}$ J. Yu, ${ }^{8}$ J. Yu, ${ }^{76}$ X. Yue, ${ }^{59 a}$ S. P. Y. Yuen, ${ }^{24}$ B. Zabinski, ${ }^{82}$ G. Zacharis, ${ }^{10}$ E. Zaffaroni, ${ }^{52}$ R. Zaidan, ${ }^{14}$ A. M. Zaitsev, ${ }^{121, p p}$ T. Zakareishvili, ${ }^{156 b}$ N. Zakharchuk, ${ }^{33}$ S. Zambito, ${ }^{57}$ D. Zanzi, ${ }^{35}$ D. R. Zaripovas, ${ }^{55}$ S. V. Zeißner, ${ }^{45}$ C. Zeitnitz, ${ }^{179}$ G. Zemaityte, ${ }^{132}$ J. C. Zeng, ${ }^{170}$ Q. Zeng, ${ }^{150}$ O. Zenin, ${ }^{121}$ D. Zerwas, ${ }^{129}$ M. Zgubič, ${ }^{132}$ D. F. Zhang, ${ }^{58 b}$ D. Zhang, ${ }^{103}$ F. Zhang, ${ }^{178}$ G. Zhang, ${ }^{58 \mathrm{a}}$ G. Zhang, ${ }^{15 b}$ H. Zhang, ${ }^{15 \mathrm{c}}$ J. Zhang, ${ }^{6}$ L. Zhang, ${ }^{15 \mathrm{c}}$ L. Zhang, ${ }^{58 \mathrm{a}}$ M. Zhang, ${ }^{170}$ P. Zhang, ${ }^{15 \mathrm{c}} \mathrm{R}$. Zhang, ${ }^{58 \mathrm{a}} \mathrm{R} . \mathrm{Zhang}^{24} \mathrm{X} . \mathrm{Zhang},{ }^{58 \mathrm{~b}}$ Y. Zhang, ${ }^{15 d}$ Z. Zhang, ${ }^{129}$ P. Zhao, ${ }^{47}$ Y. Zhao, ${ }^{58 b, 129, b b}$ Z. Zhao, ${ }^{58 a}$ A. Zhemchugov, ${ }^{77}$ Z. Zheng, ${ }^{103}$ D. Zhong, ${ }^{170}$ B. Zhou, ${ }^{103}$ C. Zhou, ${ }^{178}$ M. S. Zhou, ${ }^{15 d}$ M. Zhou, ${ }^{152}$ N. Zhou, ${ }^{58 c}$ Y. Zhou, ${ }^{7}$ C. G. Zhu, ${ }^{58 b}$ H. L. Zhu, ${ }^{58 a}$ H. Zhu, ${ }^{15 a}$ J. Zhu, ${ }^{103}$ Y. Zhu, ${ }^{58 a}$ X. Zhuang, ${ }^{15 a}$ K. Zhukov, ${ }^{108}$ V. Zhulanov, ${ }^{120 b, 120 a}$ A. Zibell, ${ }^{174}$ D. Zieminska, ${ }^{63}$ N. I. Zimine, ${ }^{77}$ S. Zimmermann, ${ }^{50}$ Z. Zinonos, ${ }^{113}$ M. Ziolkowski, ${ }^{148}$ G. Zobernig, ${ }^{178}$ A. Zoccoli, ${ }^{23 b, 23 a}$ K. Zoch, ${ }^{51}$ T. G. Zorbas, ${ }^{146}$ R. Zou, ${ }^{36}$ M. Zur Nedden, ${ }^{19}$ and L. Zwalinski ${ }^{35}$

(ATLAS Collaboration)

\author{
${ }^{1}$ Department of Physics, University of Adelaide, Adelaide, Australia \\ ${ }^{2}$ Physics Department, SUNY Albany, Albany, New York, USA \\ ${ }^{3}$ Department of Physics, University of Alberta, Edmonton, Alberta, Canada \\ ${ }^{4 a}$ Department of Physics, Ankara University, Ankara, Turkey \\ ${ }^{4 \mathrm{~b}}$ Istanbul Aydin University, Istanbul, Turkey \\ ${ }^{4 \mathrm{c}}$ Division of Physics, TOBB University of Economics and Technology, Ankara, Turkey \\ ${ }^{5}$ LAPP, Université Grenoble Alpes, Université Savoie Mont Blanc, CNRS/IN2P3, Annecy, France \\ ${ }^{6}$ High Energy Physics Division, Argonne National Laboratory, Argonne, Illinois, USA \\ ${ }^{7}$ Department of Physics, University of Arizona, Tucson, Arizona, USA \\ ${ }^{8}$ Department of Physics, University of Texas at Arlington, Arlington, Texas, USA \\ ${ }^{9}$ Physics Department, National and Kapodistrian University of Athens, Athens, Greece \\ ${ }^{10}$ Physics Department, National Technical University of Athens, Zografou, Greece \\ ${ }^{11}$ Department of Physics, University of Texas at Austin, Austin, Texas, USA \\ ${ }^{12 \mathrm{a}}$ Bahcesehir University, Faculty of Engineering and Natural Sciences, Istanbul, Turkey \\ ${ }^{12 \mathrm{~b}}$ Istanbul Bilgi University, Faculty of Engineering and Natural Sciences, Istanbul, Turkey \\ ${ }^{12 \mathrm{c}}$ Department of Physics, Bogazici University, Istanbul, Turkey \\ ${ }^{12 \mathrm{~d} D e p a r t m e n t}$ of Physics Engineering, Gaziantep University, Gaziantep, Turkey \\ ${ }^{13}$ Institute of Physics, Azerbaijan Academy of Sciences, Baku, Azerbaijan \\ ${ }^{14}$ Institut de Física d'Altes Energies (IFAE), Barcelona Institute of Science and Technology, \\ Barcelona, Spain \\ ${ }^{15 a}$ Institute of High Energy Physics, Chinese Academy of Sciences, Beijing, China \\ ${ }^{15 \mathrm{~b}}$ Physics Department, Tsinghua University, Beijing, China \\ ${ }^{15 \mathrm{c}}$ Department of Physics, Nanjing University, Nanjing, China \\ ${ }^{15 \mathrm{~d}}$ University of Chinese Academy of Science (UCAS), Beijing, China \\ ${ }^{16}$ Institute of Physics, University of Belgrade, Belgrade, Serbia \\ ${ }^{17}$ Department for Physics and Technology, University of Bergen, Bergen, Norway \\ ${ }^{18}$ Physics Division, Lawrence Berkeley National Laboratory and University of California, Berkeley, \\ California, USA \\ ${ }^{19}$ Institut für Physik, Humboldt Universität zu Berlin, Berlin, Germany \\ ${ }^{20}$ Albert Einstein Center for Fundamental Physics and Laboratory for High Energy Physics, \\ University of Bern, Bern, Switzerland \\ ${ }^{21}$ School of Physics and Astronomy, University of Birmingham, Birmingham, United Kingdom \\ ${ }^{22}$ Centro de Investigaciónes, Universidad Antonio Nariño, Bogota, Colombia \\ ${ }^{23 a}$ Dipartimento di Fisica e Astronomia, Università di Bologna, Bologna, Italy \\ ${ }^{23 \mathrm{~b}}$ INFN Sezione di Bologna, Italy \\ ${ }^{24}$ Physikalisches Institut, Universität Bonn, Bonn, Germany \\ ${ }^{25}$ Department of Physics, Boston University, Boston, Massachusetts, USA \\ ${ }^{26}$ Department of Physics, Brandeis University, Waltham, Massachusetts, USA \\ ${ }^{27 a}$ Transilvania University of Brasov, Brasov, Romania \\ ${ }^{27 \mathrm{~b}}$ Horia Hulubei National Institute of Physics and Nuclear Engineering, Bucharest, Romania \\ ${ }^{27 \mathrm{c}}$ Department of Physics, Alexandru Ioan Cuza University of Iasi, Iasi, Romania \\ ${ }^{27 \mathrm{~d}}$ National Institute for Research and Development of Isotopic and Molecular Technologies, \\ Physics Department, Cluj-Napoca, Romania
}


${ }^{27 \mathrm{e}}$ University Politehnica Bucharest, Bucharest, Romania

${ }^{27 \mathrm{f}}$ West University in Timisoara, Timisoara, Romania

${ }^{28 a}$ Faculty of Mathematics, Physics and Informatics, Comenius University, Bratislava, Slovak Republic

${ }^{28 \mathrm{~b}}$ Department of Subnuclear Physics, Institute of Experimental Physics of the Slovak Academy of Sciences, Kosice, Slovak Republic

${ }^{29}$ Physics Department, Brookhaven National Laboratory, Upton, New York, USA

${ }^{30}$ Departamento de Física, Universidad de Buenos Aires, Buenos Aires, Argentina

${ }^{31}$ Cavendish Laboratory, University of Cambridge, Cambridge, United Kingdom

${ }^{32 a}$ Department of Physics, University of Cape Town, Cape Town, South Africa

${ }^{32 \mathrm{~b}}$ Department of Mechanical Engineering Science, University of Johannesburg, Johannesburg, South Africa

${ }^{32 \mathrm{c}}$ School of Physics, University of the Witwatersrand, Johannesburg, South Africa

${ }^{33}$ Department of Physics, Carleton University, Ottawa, Ontario, Canada

${ }^{34 a}$ Faculté des Sciences Ain Chock, Réseau Universitaire de Physique des Hautes Energies-Université Hassan II, Casablanca, Morocco

${ }^{34 \mathrm{~b}}$ Centre National de l'Energie des Sciences Techniques Nucleaires (CNESTEN), Rabat, Morocco

${ }^{34 \mathrm{c}}$ Faculté des Sciences Semlalia, Université Cadi Ayyad, LPHEA-Marrakech, Morocco

${ }^{34 \mathrm{~d}}$ Faculté des Sciences, Université Mohamed Premier and LPTPM, Oujda, Morocco

${ }^{34 \mathrm{e}}$ Faculté des sciences, Université Mohammed V, Rabat, Morocco

${ }^{35}$ CERN, Geneva, Switzerland

${ }^{36}$ Enrico Fermi Institute, University of Chicago, Chicago Illinois, USA

${ }^{37}$ LPC, Université Clermont Auvergne, CNRS/IN2P3, Clermont-Ferrand, France

${ }^{38}$ Nevis Laboratory, Columbia University, Irvington, New York, USA

${ }^{39}$ Niels Bohr Institute, University of Copenhagen, Copenhagen, Denmark

${ }^{40 a}$ Dipartimento di Fisica, Università della Calabria, Rende, Italy

${ }^{40 \mathrm{~b}}$ INFN Gruppo Collegato di Cosenza, Laboratori Nazionali di Frascati, Italy

${ }^{41}$ Physics Department, Southern Methodist University, Dallas, Texas, USA

${ }^{42}$ Physics Department, University of Texas at Dallas, Richardson, Texas, USA

${ }^{43 a}$ Department of Physics, Stockholm University, Sweden

${ }^{43 \mathrm{~b}}$ Oskar Klein Centre, Stockholm, Sweden

${ }^{44}$ Deutsches Elektronen-Synchrotron DESY, Hamburg and Zeuthen, Germany

${ }^{45}$ Lehrstuhl für Experimentelle Physik IV, Technische Universität Dortmund, Dortmund, Germany

${ }^{46}$ Institut für Kern- und Teilchenphysik, Technische Universität Dresden, Dresden, Germany

${ }^{47}$ Department of Physics, Duke University, Durham, North Carolina, USA

${ }^{48}$ SUPA-School of Physics and Astronomy, University of Edinburgh, Edinburgh, United Kingdom

${ }^{49}$ INFN e Laboratori Nazionali di Frascati, Frascati, Italy

${ }^{50}$ Physikalisches Institut, Albert-Ludwigs-Universität Freiburg, Freiburg, Germany

${ }^{51}$ II. Physikalisches Institut, Georg-August-Universität Göttingen, Göttingen, Germany

${ }^{52}$ Département de Physique Nucléaire et Corpusculaire, Université de Genève, Genève, Switzerland

${ }^{53 a}$ Dipartimento di Fisica, Università di Genova, Genova, Italy

${ }^{53 \mathrm{~b}}$ INFN Sezione di Genova, Italy

${ }^{54}$ II. Physikalisches Institut, Justus-Liebig-Universität Giessen, Giessen, Germany

${ }^{55}$ SUPA-School of Physics and Astronomy, University of Glasgow, Glasgow, United Kingdom

${ }^{56}$ LPSC, Université Grenoble Alpes, CNRS/IN2P3, Grenoble INP, Grenoble, France

${ }^{57}$ Laboratory for Particle Physics and Cosmology, Harvard University, Cambridge, Massachusetts, USA

${ }^{58 \mathrm{a}}$ Department of Modern Physics and State Key Laboratory of Particle Detection and Electronics,

University of Science and Technology of China, Hefei, China

${ }^{58 \mathrm{~b}}$ Institute of Frontier and Interdisciplinary Science and Key Laboratory of Particle Physics and Particle Irradiation (MOE), Shandong University, Qingdao, China

${ }^{58 c}$ School of Physics and Astronomy, Shanghai Jiao Tong University, KLPPAC-MoE, SKLPPC,

Shanghai, China

${ }^{58 \mathrm{~d}}$ Tsung-Dao Lee Institute, Shanghai, China

${ }^{59 a}$ Kirchhoff-Institut für Physik, Ruprecht-Karls-Universität Heidelberg, Heidelberg, Germany

${ }^{59 \mathrm{~b}}$ Physikalisches Institut, Ruprecht-Karls-Universität Heidelberg, Heidelberg, Germany

${ }^{60}$ Faculty of Applied Information Science, Hiroshima Institute of Technology, Hiroshima, Japan

${ }^{61 a}$ Department of Physics, Chinese University of Hong Kong, Shatin, N.T., Hong Kong, China

${ }^{61 \mathrm{~b}}$ Department of Physics, University of Hong Kong, Hong Kong, China

${ }^{61 \mathrm{c}}$ Department of Physics and Institute for Advanced Study, Hong Kong University of Science and Technology, Clear Water Bay, Kowloon, Hong Kong, China

${ }^{62}$ Department of Physics, National Tsing Hua University, Hsinchu, Taiwan 
${ }^{63}$ Department of Physics, Indiana University, Bloomington, Indiana, USA

${ }^{64 \mathrm{a}}$ INFN Gruppo Collegato di Udine, Sezione di Trieste, Udine, Italy

${ }^{64 \mathrm{~b}}$ ICTP, Trieste, Italy

${ }^{64 \mathrm{c}}$ Dipartimento di Chimica, Fisica e Ambiente, Università di Udine, Udine, Italy

${ }^{65 \mathrm{a}}$ INFN Sezione di Lecce, Italy

${ }^{65 \mathrm{~b}}$ Dipartimento di Matematica e Fisica, Università del Salento, Lecce, Italy

${ }^{66 a}$ INFN Sezione di Milano, Italy

${ }^{66 \mathrm{~b}}$ Dipartimento di Fisica, Università di Milano, Milano, Italy

${ }^{67 a}$ INFN Sezione di Napoli, Italy

${ }^{67 \mathrm{~b}}$ Dipartimento di Fisica, Università di Napoli, Napoli, Italy

${ }^{68 \mathrm{a}}$ INFN Sezione di Pavia, Italy

${ }^{68 \mathrm{~b}}$ Dipartimento di Fisica, Università di Pavia, Pavia, Italy

${ }^{69 a}$ INFN Sezione di Pisa, Italy

${ }^{69 b}$ Dipartimento di Fisica E. Fermi, Università di Pisa, Pisa, Italy

${ }^{70 a}$ INFN Sezione di Roma, Italy

${ }^{70 \mathrm{~b}}$ Dipartimento di Fisica, Sapienza Università di Roma, Roma, Italy

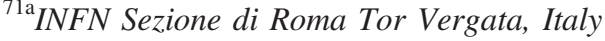

${ }^{71 \mathrm{~b}}$ Dipartimento di Fisica, Università di Roma Tor Vergata, Roma, Italy

${ }^{72 \mathrm{a}}$ INFN Sezione di Roma Tre, Italy

${ }^{72 \mathrm{~b}}$ Dipartimento di Matematica e Fisica, Università Roma Tre, Roma, Italy

${ }^{73 a}$ INFN-TIFPA, Italy

${ }^{73 \mathrm{~b}}$ Università degli Studi di Trento, Trento, Italy

${ }^{74}$ Institut für Astro- und Teilchenphysik, Leopold-Franzens-Universität, Innsbruck, Austria

${ }^{75}$ University of Iowa, Iowa City, Iowa, USA

${ }^{76}$ Department of Physics and Astronomy, Iowa State University, Ames, Iowa, USA

${ }^{77}$ Joint Institute for Nuclear Research, Dubna, Russia

${ }^{78 a}$ Departamento de Engenharia Elétrica, Universidade Federal de Juiz de Fora (UFJF),

Juiz de Fora, Brazil

${ }^{78 b}$ Universidade Federal do Rio De Janeiro COPPE/EE/IF, Rio de Janeiro, Brazil

${ }^{78 c}$ Universidade Federal de São João del Rei (UFSJ), São João del Rei, Brazil

${ }^{78 d}$ Instituto de Física, Universidade de São Paulo, São Paulo, Brazil

${ }^{79}$ KEK, High Energy Accelerator Research Organization, Tsukuba, Japan

${ }^{80}$ Graduate School of Science, Kobe University, Kobe, Japan

${ }^{81 \mathrm{a}}$ AGH University of Science and Technology, Faculty of Physics and Applied Computer Science, Krakow, Poland

${ }^{81 \mathrm{~b}}$ Marian Smoluchowski Institute of Physics, Jagiellonian University, Krakow, Poland

${ }^{82}$ Institute of Nuclear Physics Polish Academy of Sciences, Krakow, Poland

${ }^{83}$ Faculty of Science, Kyoto University, Kyoto, Japan

${ }^{84}$ Kyoto University of Education, Kyoto, Japan

${ }^{85}$ Research Center for Advanced Particle Physics and Department of Physics, Kyushu University, Fukuoka, Japan

${ }^{86}$ Instituto de Física La Plata, Universidad Nacional de La Plata and CONICET, La Plata, Argentina

${ }^{87}$ Physics Department, Lancaster University, Lancaster, United Kingdom

${ }^{88}$ Oliver Lodge Laboratory, University of Liverpool, Liverpool, United Kingdom

${ }^{89}$ Department of Experimental Particle Physics, Jožef Stefan Institute and Department of Physics, University of Ljubljana, Ljubljana, Slovenia

${ }^{90}$ School of Physics and Astronomy, Queen Mary University of London, London, United Kingdom

${ }^{91}$ Department of Physics, Royal Holloway University of London, Egham, United Kingdom

${ }^{92}$ Department of Physics and Astronomy, University College London, London, United Kingdom

${ }^{93}$ Louisiana Tech University, Ruston, Los Angeles, USA

${ }^{94}$ Fysiska institutionen, Lunds universitet, Lund, Sweden

${ }^{95}$ Centre de Calcul de l'Institut National de Physique Nucléaire et de Physique des Particules (IN2P3), Villeurbanne, France

${ }^{96}$ Departamento de Física Teorica C-15 and CIAFF, Universidad Autónoma de Madrid, Madrid, Spain

${ }^{97}$ Institut für Physik, Universität Mainz, Mainz, Germany

${ }^{98}$ School of Physics and Astronomy, University of Manchester, Manchester, United Kingdom

${ }^{99}$ CPPM, Aix-Marseille Université, CNRS/IN2P3, Marseille, France

${ }^{100}$ Department of Physics, University of Massachusetts, Amherst, Massachusetts, USA

${ }^{101}$ Department of Physics, McGill University, Montreal, Quebec, Canada

${ }^{102}$ School of Physics, University of Melbourne, Victoria, Australia 
${ }^{103}$ Department of Physics, University of Michigan, Ann Arbor, Michigan, USA

${ }^{104}$ Department of Physics and Astronomy, Michigan State University, East Lansing, Michigan, USA

${ }^{105}$ B.I. Stepanov Institute of Physics, National Academy of Sciences of Belarus, Minsk, Belarus

${ }^{106}$ Research Institute for Nuclear Problems of Byelorussian State University, Minsk, Belarus

${ }^{107}$ Group of Particle Physics, University of Montreal, Montreal, Quebec, Canada

${ }^{108}$ P.N. Lebedev Physical Institute of the Russian Academy of Sciences, Moscow, Russia

${ }^{109}$ Institute for Theoretical and Experimental Physics of the National Research Centre Kurchatov Institute,

Moscow, Russia

${ }^{110}$ National Research Nuclear University MEPhI, Moscow, Russia

${ }^{111}$ D.V. Skobeltsyn Institute of Nuclear Physics, M.V. Lomonosov Moscow State University,

Moscow, Russia

${ }^{112}$ Fakultät für Physik, Ludwig-Maximilians-Universität München, München, Germany

${ }^{113}$ Max-Planck-Institut für Physik (Werner-Heisenberg-Institut), München, Germany

${ }^{114}$ Nagasaki Institute of Applied Science, Nagasaki, Japan

${ }^{115}$ Graduate School of Science and Kobayashi-Maskawa Institute, Nagoya University, Nagoya, Japan

${ }^{116}$ Department of Physics and Astronomy, University of New Mexico, Albuquerque, New Mexico, USA

${ }^{117}$ Institute for Mathematics, Astrophysics and Particle Physics, Radboud University Nijmegen/Nikhef, Nijmegen, Netherlands

${ }^{118}$ Nikhef National Institute for Subatomic Physics and University of Amsterdam, Amsterdam, Netherlands

${ }^{119}$ Department of Physics, Northern Illinois University, DeKalb, Illinois, USA

${ }^{120 a}$ Budker Institute of Nuclear Physics and NSU, SB RAS, Novosibirsk, Russia

${ }^{120 \mathrm{~b}}$ Novosibirsk State University Novosibirsk, Russia

${ }^{121}$ Institute for High Energy Physics of the National Research Centre Kurchatov Institute, Protvino, Russia

${ }^{122}$ Department of Physics, New York University, New York, New York, USA

${ }^{123}$ Ohio State University, Columbus, Ohio, USA

${ }^{124}$ Faculty of Science, Okayama University, Okayama, Japan

${ }^{125}$ Homer L. Dodge Department of Physics and Astronomy, University of Oklahoma, Norman, Oklahoma, USA

${ }^{126}$ Department of Physics, Oklahoma State University, Stillwater, Oklahoma, USA

${ }^{127}$ Palacký University, RCPTM, Joint Laboratory of Optics, Olomouc, Czech Republic

${ }^{128}$ Center for High Energy Physics, University of Oregon, Eugene, Oregon, USA

${ }^{129}$ LAL, Université Paris-Sud, CNRS/IN2P3, Université Paris-Saclay, Orsay, France

${ }^{130}$ Graduate School of Science, Osaka University, Osaka, Japan

${ }^{131}$ Department of Physics, University of Oslo, Oslo, Norway

${ }^{132}$ Department of Physics, Oxford University, Oxford, United Kingdom

${ }^{133}$ LPNHE, Sorbonne Université, Paris Diderot Sorbonne Paris Cité, CNRS/IN2P3, Paris, France

${ }^{134}$ Department of Physics, University of Pennsylvania, Philadelphia, Pennsylvania, USA

${ }^{135}$ Konstantinov Nuclear Physics Institute of National Research Centre "Kurchatov Institute”, PNPI, St. Petersburg, Russia

${ }^{136}$ Department of Physics and Astronomy, University of Pittsburgh, Pittsburgh, Pennsylvania, USA

${ }^{137 a}$ Laboratório de Instrumentação e Física Experimental de Partículas-LIP, Portugal

${ }^{137 b}$ Departamento de Física, Faculdade de Ciências, Universidade de Lisboa, Lisboa, Portugal

${ }^{137 \mathrm{c}}$ Departamento de Física, Universidade de Coimbra, Coimbra, Portugal

${ }^{137 \mathrm{~d}}$ Centro de Física Nuclear da Universidade de Lisboa, Lisboa, Portugal

${ }^{137 e}$ Departamento de Física, Universidade do Minho, Braga, Portugal

${ }^{137 \mathrm{f}}$ Departamento de Física Teorica y del Cosmos, Universidad de Granada, Granada (Spain), Spain

${ }^{137 \mathrm{~g}}$ Dep Física and CEFITEC of Faculdade de Ciências e Tecnologia, Universidade Nova de Lisboa, Caparica, Portugal

${ }^{138}$ Institute of Physics of the Czech Academy of Sciences, Prague, Czech Republic

${ }^{139}$ Czech Technical University in Prague, Prague, Czech Republic

${ }^{140}$ Charles University, Faculty of Mathematics and Physics, Prague, Czech Republic

${ }^{141}$ Particle Physics Department, Rutherford Appleton Laboratory, Didcot, United Kingdom

${ }^{142}$ IRFU, CEA, Université Paris-Saclay, Gif-sur-Yvette, France

${ }^{143}$ Santa Cruz, Institute for Particle Physics, University of California Santa Cruz, Santa Cruz, California, USA

${ }^{144 a}$ Departamento de Física, Pontificia Universidad Católica de Chile, Santiago, Chile

${ }^{144 b}$ Departamento de Física, Universidad Técnica Federico Santa María, Valparaíso, Chile

${ }^{145}$ Department of Physics, University of Washington, Seattle, Washington, USA

${ }^{146}$ Department of Physics and Astronomy, University of Sheffield, Sheffield, United Kingdom

${ }^{147}$ Department of Physics, Shinshu University, Nagano, Japan 


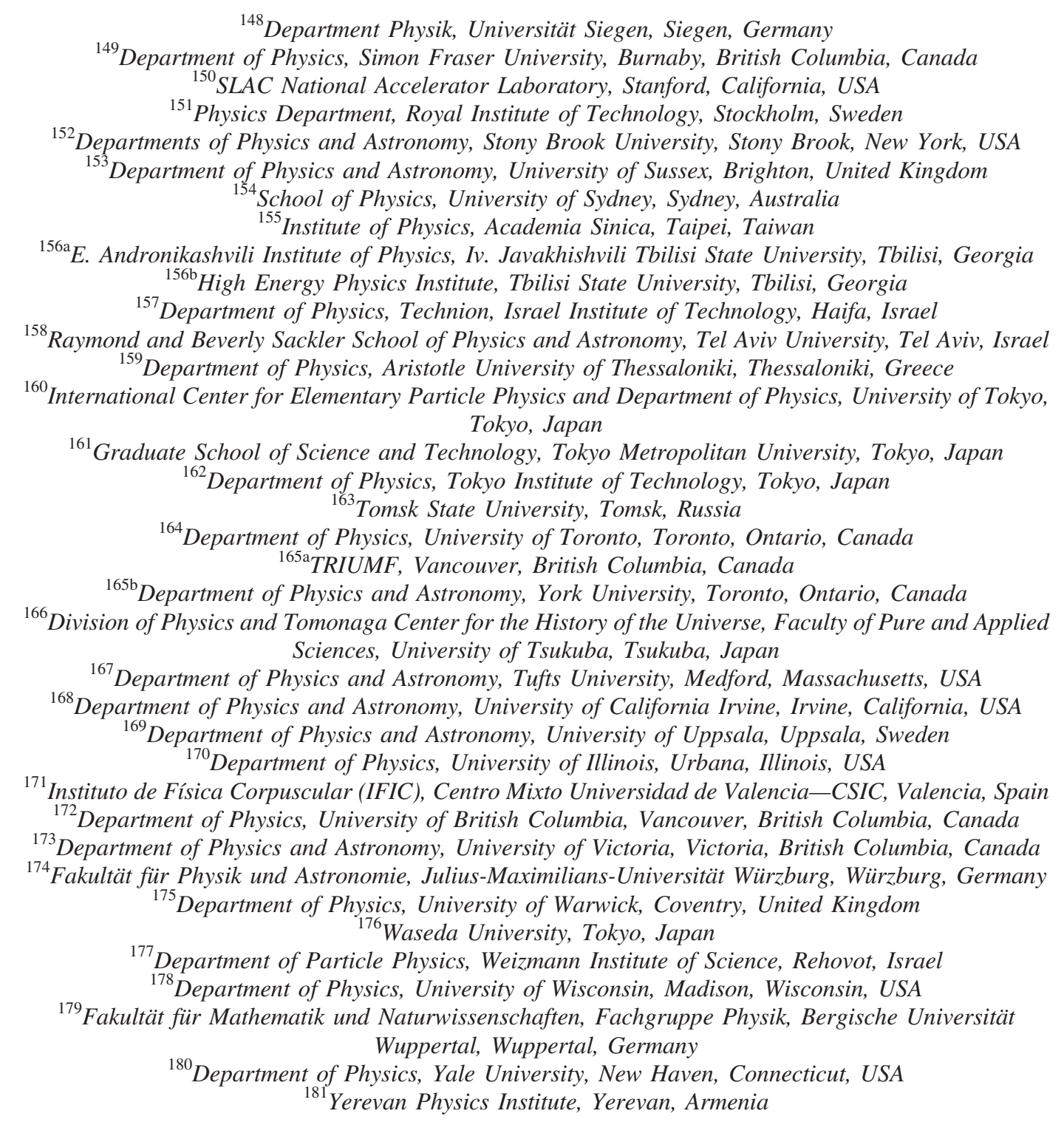

${ }^{\mathrm{a}}$ Deceased.

${ }^{\mathrm{b}}$ Also at Department of Physics, King's College London, London, United Kingdom.

${ }^{\mathrm{c}}$ Also at Istanbul University, Dept. of Physics, Istanbul, Turkey.

${ }^{\mathrm{d}}$ Also at Instituto de Física Teórica de la Universidad Autónoma de Madrid, Spain.

${ }^{\mathrm{e}}$ Also at Institute of Physics, Azerbaijan Academy of Sciences, Baku, Azerbaijan.

${ }^{\mathrm{f}}$ Also at TRIUMF, Vancouver, British Columbia, Canada.

${ }^{g}$ Also at Department of Physics and Astronomy, University of Louisville, Louisville, Kentucky, USA.

${ }^{\mathrm{h}}$ Also at Department of Physics, University of Fribourg, Fribourg, Switzerland.

${ }^{\mathrm{i}}$ Also at Physics Dept, University of South Africa, Pretoria, South Africa.

${ }^{\mathrm{j}}$ Also at Departament de Fisica de la Universitat Autonoma de Barcelona, Barcelona, Spain.

${ }^{\mathrm{k}}$ Also at Tomsk State University, Tomsk, and Moscow Institute of Physics and Technology State University, Dolgoprudny, Russia.

${ }^{1}$ Also at The Collaborative Innovation Center of Quantum Matter (CICQM), Beijing, China.

${ }^{\mathrm{m}}$ Also at Departamento de Física, Instituto Superior Técnico, Universidade de Lisboa, Lisboa, Portugal.

${ }^{\mathrm{n}}$ Also at Universita di Napoli Parthenope, Napoli, Italy.

${ }^{\circ}$ Also at Institute of Particle Physics (IPP), Canada.

${ }^{\mathrm{p}}$ Also at II. Physikalisches Institut, Georg-August-Universität Göttingen, Göttingen, Germany.

${ }^{\mathrm{q}}$ Also at Horia Hulubei National Institute of Physics and Nuclear Engineering, Bucharest, Romania.

${ }^{\mathrm{r}}$ Also at CPPM, Aix-Marseille Université, CNRS/IN2P3, Marseille, France.

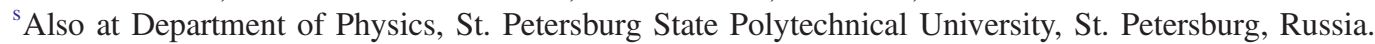

${ }^{\mathrm{t}}$ Also at Borough of Manhattan Community College, City University of New York,, New York, USA. 
uAlso at Department of Physics, California State University, Fresno, California, USA.

${ }^{\mathrm{v}}$ Also at Department of Financial and Management Engineering, University of the Aegean, Chios, Greece.

${ }^{\text {w}}$ Also at Centre for High Performance Computing, CSIR Campus, Rosebank, Cape Town, South Africa.

${ }^{\mathrm{x}}$ Also at Louisiana Tech University, Ruston, Los Angeles, USA.

${ }^{\mathrm{y}}$ Also at California State University, East Bay, USA.

${ }^{\mathrm{z}}$ Also at Institucio Catalana de Recerca i Estudis Avancats, ICREA, Barcelona, Spain.

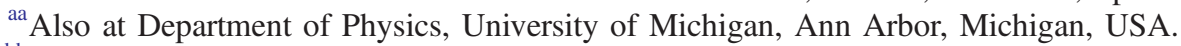

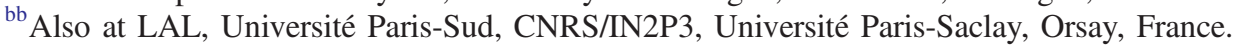

${ }^{c c}$ Also at Graduate School of Science, Osaka University, Osaka, Japan.

${ }^{\mathrm{dd}}$ Also at Physikalisches Institut, Albert-Ludwigs-Universität Freiburg, Freiburg, Germany.

${ }^{e e}$ Also at Institute for Mathematics, Astrophysics and Particle Physics, Radboud University Nijmegen/Nikhef, Nijmegen, Netherlands.

${ }^{\mathrm{ff}}$ Also at Institute of Theoretical Physics, Ilia State University, Tbilisi, Georgia.

${ }^{\mathrm{gg}}$ Also at CERN, Geneva, Switzerland.

${ }^{\text {hh }}$ Also at Department of Physics, Stanford University, USA.

${ }^{\text {ii } A l s o ~ a t ~ M a n h a t t a n ~ C o l l e g e, ~ N e w ~ Y o r k, ~ N e w ~ Y o r k, ~ U S A . ~}$

${ }^{\mathrm{jj}}$ Also at Joint Institute for Nuclear Research, Dubna, Russia.

${ }^{\mathrm{kk}}$ Also at Hellenic Open University, Patras, Greece.

${ }^{11}$ Also at LPNHE, Sorbonne Université, Paris Diderot Sorbonne Paris Cité, CNRS/IN2P3, Paris, France.

${ }^{\mathrm{mm}}$ Also at The City College of New York, New York, New York, USA.

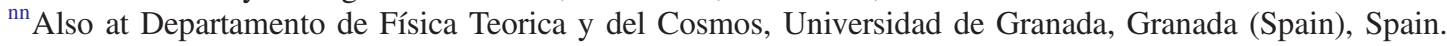

${ }^{\text {oo }}$ Also at Department of Physics, California State University, Sacramento, California, USA.

pp Also at Moscow Institute of Physics and Technology State University, Dolgoprudny, Russia.

${ }^{\mathrm{qq}}$ Also at Département de Physique Nucléaire et Corpusculaire, Université de Genève, Genève, Switzerland.

${ }^{\mathrm{rr}}$ Also at Department of Physics and Astronomy, University of Sheffield, Sheffield, United Kingdom.

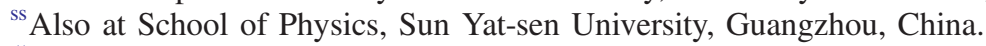

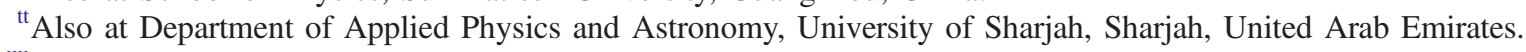

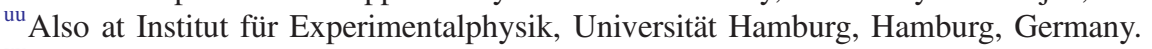

${ }^{\mathrm{vv}}$ Also at National Research Nuclear University MEPhI, Moscow, Russia.

${ }^{w w}$ Also at Institute for Particle and Nuclear Physics, Wigner Research Centre for Physics, Budapest, Hungary.

${ }^{\mathrm{xx}}$ Also at Giresun University, Faculty of Engineering, Giresun, Turkey.

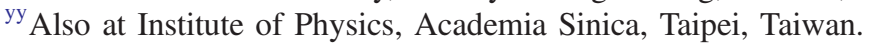

